\title{
Abstracts: Free Communications
}

Helen Bayne

Affiliation: High Performance Centre, University of Pretoria

\section{Alteration of Biomechanical Risk Factors For Low Back Injury: a Coaching Intervention}

Introduction: Lateral flexion of the trunk after front foot contact during the bowling delivery stride is suggested to be a key mechanical component of lumbar spondylolysis aetiology in cricket fast bowlers [1]. Certain aspects of the bowling action have been shown to be changeable over longterm (>2 year) coaching interventions, but it is believed that lateral flexion is difficult to change [2,3]. The current study aimed to investigate the efficacy of a coaching intervention intended to reduce trunk lateral flexion.

Methods: Eight fast bowlers, aged $17-19$ years, underwent high-speed $(120 \mathrm{~Hz})$ video analysis of their bowling technique. Trunk lateral flexion at ball release was measured as the angle between the vertical and a line from the umbilicus to the sternal notch. Five bowlers were identified as highrisk (trunk lateral flexion greater than $40^{\circ}$ at ball release) and were assigned to a 12-week coaching intervention with the focus on reducing lateral flexion. The video analysis was repeated following the intervention period. Test-retest reliability of the measurement was examined using the intraclass correlation coefficient, which was good $(\mathrm{ICC}=0.93)$.

Results: Following the coaching intervention, the trunk lateral flexion angle was reduced in each bowler and there was a statistically significant difference between the pre-and post-intervention group mean (pre-intervention: $52^{\circ} \pm 5$; post-intervention: $45^{\circ} \pm 4 ; t=3.98 ; p<0.05$ ).

Discussion: In order to prevent injury, interventions need to target modifiable risk factors and mechanisms. The fast bowling action is a complex motor skill, and there is a commonly held belief that significant changes in technique are difficult to attain. A previous study found that the lower trunk lateral flexion angle was not improved, despite a 2-year coaching focus on this aspect of the bowling action. In the current study, trunk lateral flexion was determined by the posture of the upper trunk, and was found to be reduced following a coaching intervention. The motion of the upper trunk may be more changeable than the lower trunk. Upper trunk lateral flexion has been associated with low back injury incidence in fast bowlers and this study suggests that it can be improved through coaching.

Daniel Mark Botha

Affiliation: Department of Sport and Movement Science, University of Johannesburg

\section{The Effect of Hyperbaric Oxygen and Blood Platelet Injection Therapy on the Healing of Hamstring Injuries in Rugby Players, a Case} Series Report

Background: The increase in frequency and severity of hamstring injuries in rugby has been well documented. There are a number of ultrastructural and immuno-histochemical studies involving hyperbaric oxygen treatment in skeletal muscle, as well as soft tissue healing. The recovery time and complete restoration of function using traditional rehabilitation modalities requires a long duration and intensive treatment, as well as extensive off-field time. Hyperbaric oxygen therapy, in conjunction with blood platelet injection therapy, serves as a valuable addition to previously known and trusted rehabilitation techniques and protocols for the healing of musculoskeletal or soft tissue injuries.

Objectives: The primary aim of this case report is to describe the effect on the recovery time of hamstring injuries when combining hyperbaric oxygen therapy (HBOT) and platelet rich plasma (PRP) injection therapy with exercise rehabilitation, compared with exercise rehabilitation alone.

Method: A retrospective, post-intervention data analysis was used in this case series report. The sample of injuries came from players from a premier division rugby team (23 players) who had sustained 42 hamstring injuries. Data pertaining to hamstring injuries and treatment, obtained through collaboration with a professional rugby union and a South African Undersea and Hyperbaric Medicine Association (SAUHMA) accredited Hyperbaric Medicine (HBOT) Centre, was analysed using computer-based Statistical Programme for the Social Sciences (SPSS) software. The significance value was set at $5 \%$.

Results: A significant decrease in the injury time of the hamstring injuries in rugby players was noted, with a $38 \%$ reduction in injury time in players with a grade-one injury, and $45.7 \%$ reduction in players with a grade-two injury. In terms of recurrent injuries, $62 \%$ of players with grade-one injuries remained uninjured after treatment, and the percentage of re-injured players with grade-two injuries was $0 \%$ after HBOT, PRP and physical therapy treatment.

Conclusion: The notion that the healing time of hamstring injuries will decrease when HBOT and PRP are administered in conjunction with traditional rehabilitation therapy is indicated by the data of this reported.

\section{Alison Brooks}

Affiliation: University of Wisconsin-Madison, USA

Additional Authors: Alison Brooks, Timothy McGuine, Scott Hetzel

\section{Effect of New Rule Limiting Full Contact Practice on Incidence of Sport Related Concussion in High School Football Players}

Purpose: To determine if the rate of sport-related concussion (SRC) is lower following state interscholastic athletic association mandated rule change (effective starting with 2014 season) that limited amount and duration of full contact activities during high school football practice sessions. The new rule prohibited full contact during week 1, and limited full contact to $75 \mathrm{~min} / \mathrm{wk}$ during week 2 and to $60 \mathrm{~min} / \mathrm{wk}$ week 3 and beyond. Full contact was defined as drills or game situations that occur at game speed when full tackles are made at a competitive pace and players are taken to the ground.

Methods: Prospective cohort study of US high school football players (grades 9-12). Prior to rule change - 2012 (36 schools), 2013 (18 schools) seasons, 2081 players (age $=15.9+1.2 \mathrm{yrs}$ ). After rule change -2014 season ( 26 schools), 945 players (age $=15.9+1.1 \mathrm{yrs}$ ). Players provided previous concussion and demographic information by self-report. Licensed Athletic Trainers recorded incidence and severity (median, IQR days lost) for each SRC. Chi-square tests were used to compare incidence of SRC in pre-rule 2012/2013 seasons with incidence in post-rule 2014 season. Wilcoxon Rank Sum tests were used to determine differences in severity (days lost) of SRC.

Results: Total of 67 players (7.1\%) sustained 70 SRC in 2014 compared to 206 players (9.0\%) who sustained 211 SRC in $2012 / 2013$ combined. Overall rate of SRC per 1000 Athletic Exposures (AE) was 1.28 in 2014 compared to 1.57 in 2012/2013 ( $p=0.155$ ). Tackling was primary mechanism of injury for $46 \%$ of all SRC. The rate of SRC sustained overall in practice was significantly lower ( $p=.003)$ post-rule in 2014 (15 SRCs, 0.33/1000AE) compared to pre-rule 2012/2013 (86 SRCs, 0.76/1000AE). For 2014 season,12/15 SRCs were sustained during full contact practices, compared to $82 / 86$ SRCs in 2012/2013 seasons. The rate of SRC in full contact practice was 0.57/1000AE in 2014 compared to $0.87 / 1000 \mathrm{AE}$ in $2012 / 2013(p=0.216)$. There was no difference $(p=0.999)$ in the rate of SRC sustained in games pre $(5.81 / 1000 \mathrm{AE})$ and post rule $(5.74 / 1000 \mathrm{AE})$ change. There was no difference $(p=0.967)$ in severity of SRC pre (13 days lost [10-18 IQR]) and post rule (14 days lost [10.25-16 IQR]). Years of football playing experience did not affect incidence of SRC in 2014 season ( $p=0.941)$.

Conclusions: The majority of SRC sustained in high school football practice occurred during full contact activities. The rate of SRC sustained in high school football practice was more than twice as high in the two seasons prior to a rule change limiting the amount and duration of full contact activities. Significance of Findings: Limitations on contact during high school football practice may be one effective measure to reduce the incidence of SRC. 
Affiliation: Division of Exercise Science and Sports Medicine, Department of Human Biology, Faculty of Health Additional authors: James Brown, Evert Verhagen, Willem Van Mechelen, Mike Lambert, Catherine Draper

\title{
Coaches' and referees' perceptions of the mandatory BokSmart safety course
}

Introduction: The South African Rugby Union (SARU) launched the BokSmart nationwide injury prevention program to reduce rugby-related catastrophic injuries. In January 2010, SARU made the BokSmart rugby safety course mandatory for all South African coaches and referees. The aim of this qualitative study was to evaluate the coaches and referees' perceptions of the implementation of BokSmart, with specific focus on the mandatory safety course.

Methods: Seven focus groups were conducted with coaches $(n=43)$ and referees $(n=7)$ : one from each socioeconomic status (SES) group (low, mid and high) and separately for school (junior) and adult (senior) age groups. These findings were compared to those of the trainers who facilitate the mandatory safety courses. The RE-AIM framework was used retrospectively to categorize the findings.

Results: Every focus group mentioned that they felt that the BokSmart program was capable of reducing catastrophic injuries in players. However, there every group also mentioned also mentioned barriers to optimal implementation of the BokSmart mandatory safety course prescriptions. Mid/low SES coaches described infrastructure-related barriers to implementing BokSmart safety course prescriptions (for e.g. not grass fields to train on), while high SES coaches felt the course to be a 'waste of their time' and that they already knew the course content. Referees appeared to be incapable of policing all BokSmart regulations. In general, most coaches, referees and trainers felt that the training course could be shorter and more practical (less classroom and more field-based).

Conclusions: The barriers identified in this study could be hindering optimal BokSmart implementation. These barriers differed by role (coach/referee) and SES of the coaches (high, mid or low). Understanding these barriers, as well as their determinants, is essential for BokSmart implementers to optimise the program.

Nicholas Burger

Affiliation: University of Cape Town

Additional Authors: Nicholas Burger, Mike I Lambert, Wayne Viljoen, James C Brown, Clint Readhead, Sharief Hendricks

\section{Mechanisms and factors associated with tackle-related injuries in high-level under-18 rugby union players}

Introduction: Rugby union carries a high injury incidence and the tackle is associated with the majority of these injuries. Detailed understanding of injury mechanisms is required to formulate injury prevention strategies. The aim of this study was to conduct video analysis of tackle-related injury events to identify the mechanisms and associated risk factors in high level youth rugby union players.

Methods: Injury data and video footage were captured at the 2011-2013 under-18 Craven Week tournaments. Based on tackle-related injury events, a representative 'control' sample of matched non-injury events in the same injured players were identified. The video footage (pre-contact, contact and post-contact) was then retrospectively analysed and coded. Forty nine tackled-related injury events and 248 non-injury events were analysed. Descriptive statistics (\%) were reported and multinomial regression analyses (STATA® version 12) are currently in progress.

Results: Tacklers were more successful when remaining uninjured $(72 \%)$ in comparison to injury events $(31 \%)$, and ball-carriers were more successful (tackle break or offload) when they remained uninjured (32\%) in comparison to injury events $(9 \%)$. Tackler were injured most often inside their own half $(77 \%)$ and ball-carriers sustained most injuries in opposition half $(78 \%)$. The majority of ball-carrier injuries ( $70 \%)$ occurred within one pass from the previous phase. The majority of injury events involved high impact forces for injured tacklers (77\%) and ball-carriers $(65 \%)$. Ballcarriers were more likely to be injured when running fast (65\%). Tacklers were less likely to be injured when running slow-moderate speed (83\%) and were at greater risk when ball-carriers were travelling fast (73\%). A ball-carrier was less likely to be injured when they were aware of impending contact (90\%), and when they exhibited moderate-strong leg-drive (65\%) and fending (62\%).

Conclusion: Improving tackle technique may reduce injury risk and may result in a successful performance outcome. Contact intensity and injury risk may increase when play approaches the try-line and attenuating impact forces may reduce injury rates. Playing the ball wide, improving attunement/awareness, strong fending and strong leg-drive may reduce risk of injury for ball-carriers. Video footage may be used to identify injury risk factors to help guide future injury intervention strategies.

\author{
Matthew Clark \\ Affiliation: Rhodes University \\ Additional Authors: Matthew Clark, Dr Candice Christie
}

\section{The Effectiveness of Constraints-led Training on Skill Development in Interceptive Sports: A Systematic Review}

Introduction: While the benefits of constraints-led training to assist in the development of technical and cognitive skill in sport appear obvious, the evidence for this type of training is not clear. Therefore, a systematic review of the literature was conducted to establish the effectiveness of this approach to training.

Methods: Four databases were searched for journal articles focused on skill acquisition within interceptive sports. A methodological and reporting quality assessment was done to determine the quality of each article. Fourteen articles were selected for review.

Results: The results of these two quality assessments revealed poor quality scores for the majority of the studies. The effectiveness of constraintsled training on skill development could not be ascertained. Key methodological structures were identified as well as aspects of methodology that should be avoided to ensure reliable results for future studies. Conclusion: Future research is required to determine the effectiveness of constraintsled training on interceptive sporting performance.

Keywords: skill acquisition, ecological dynamics approach, training, sports science, review 


\section{Job-related barriers encountered by football coaches in Gauteng Province of South Africa}

Background: Football is the most played sport in the world and South Africa, in particular. Despite its laudable acclaim, research has shown that there is a high turnover of football coaches in the country. Therefore, this study was designed to investigate work-related barriers encountered by such coaches in the Gauteng Province of South Africa.

Methods: Eighty six football coaches were purposively recruited to participate in this study. These coaches completed a 34-item questionnaire on the perceived hindrances to coaching which yielded a reliability coefficient of 0.952 .

Results: Results indicated that football coaches identified lack of support for women players and coaches, unfair treatment to women, inadequate salary and lack of opportunities for promotion as major barriers to their coaching. Pressure to win, lack of time, unfavourable working hours and family commitments were reported as the least barriers encountered by coaches.

Conclusion: It is recommended that the South African Football Association (SAFA) should take cognizance of these findings and develop remedial measures to alleviate the challenges facing football coaches. The association should also ensure that all forms of discrimination against female coaches are eliminated and that their needs are well catered for.

Ashleigh De Freitas

Affiliation: UKZN

Additional Authors: Ashleigh De Freitas, Rowena Naidoo

\section{Female Athlete Triad Risk Stratification in KwaZulu-Natal Elite Sprint and Distance Swimmers}

Introduction: The Female Athlete Triad (FAT) is a syndrome that poses a serious threat to the health status of physically active females. It comprises of three interconnected components namely; low energy availability (with or without disordered eating) that occurs due to insufficient calorie intake in combination with high amounts of physical activity, menstrual dysfunctions defined as exercise induced menstrual dysfunction and low bone mineral density (BMD) in which the bone mineral density is weakened as a result of prolonged menstrual dysfunction. These components are linked across a continuum of healthy (optimal energy availability, eumenorrhea (a menstrual cycle of twenty eight days), and optimal bone health) to unhealthy. Method(s): Twenty-one National Level KwaZulu-Natal sprint $(n=11)$ and distance $(n=10)$ swimmers participated in this descriptive cross-sectional study. For descriptive purposes, anthropometries measurements (weight, height, skin folds and BMI) were recorded. Each participant completed three eating disorder questionnaires (Eating Attitude Test (EAT-26), Body shape Questionnaire (BSQ-34) and a Bulimic Investigatory Test, Edinburgh (BITE)), a Menstrual Cycle and Time Spent in Exercise Questionnaire and a Self-Administered Bone Mineral Density questionnaire. A Bioelectrical Impedance Device was used to measure full body composition. The participants also completed an online ASA24 Dietary Recall of the previous day's food and drink intake. The criteria for the risk of the FAT was determined by a positive score for all three FAT components. Result(s): Significance will be set at a $p \leq 0.05$. The comparison between the different sporting disciplines revealed that sprint swimmers are more at risk (63.6\%) for disordered eating compared to distance swimmers $(50 \%)$. Low energy availability was significantly evident in sprint swimmers with a result of 9.79 kcal.kg-1FFM.d-1 (SD 9.56) $(P=.005)$. Only six $(28.57 \%)$ swimmers in total were classified as having a menstrual dysfunction. Sprint swimmers showed to have a $90.9 \%$ risk of low BMD. The overall results signified that $9.5 \%$ were not at risk, $14.3 \%$ showed a low risk, $52.4 \%$ had a moderate risk and $23.8 \%$ revealed as having a high risk for the FAT. Conclusion: Elite sprint and distance swimmers are not at risk for the FAT, however, elite level sprint and distance swimmers are susceptible to the risk of FAT components.

Jaymie Donaldson

Affiliation: Discipline of Biokinetics, Exercise and Leisure Sciences, University of Kwazulu-Natal

Additional authors: Jaymie Donaldson, Michael Ormsbee, Andrew McKune

\section{The Effect of Protein Ingestion Before Sleep on Post Exercise Overnight Recovery and Performance in Athletes}

Introduction: Recent research has shown that the provision of dietary protein before sleep leads to enhanced dietary protein digestion and absorption, thereby increasing plasma amino acid availability. In addition it has been shown that the increase in plasma amino acid availability throughout the night stimulates protein synthesis and attenuates protein breakdown, thereby improving protein balance during overnight recovery from exercise. Currently there is limited information on whether the stimulation of protein synthesis results in improved performance and recovery the following day in physically active individuals. Aim: To investigate the effect of protein supplementation (PRO) and a placebo (PLA) before sleep, post a bout of resistance exercise, on performance and recovery the following day in athletes. Methods: Fifteen male, resistance-trained athletes were recruited for this randomized, double - blind, placebo-controlled study (PRO: $n=9 ; 24.9 \pm 1.8$ years; $180.2 \pm 2.8 \mathrm{~cm} ; 84.5 \pm 3.3 \mathrm{~kg})(P L A$ : $n=6$; $28.7 \pm 4.3$ years; $180.1 \pm 3.7 \mathrm{~cm} ; 87.6 \pm 5.2 \mathrm{~kg}$ ). Participants performed a strenuous 45 -minute resistance exercise bout consisting of 8 sets of 8 repetitions of both squat and bench press at $75 \%$ of their calculated $1 \mathrm{RM}$ for each (19h15). Thirty minutes prior to sleep (21h00) they consumed either $40 \mathrm{~g}$ casein protein or placebo. Venous blood samples were obtained pre exercise (T1) (18:30), immediately post (T2) (20h00), 1 hour post (T3) exercise at $21 \mathrm{~h} 00$ and at $08 \mathrm{h00}$ (T4) the next morning to measure creatine kinase (CK) and C-reactive protein (CRP). Peak muscle velocity and power output, maximal upper and lower body strength were measured at T1, T2 and the next morning at 08h30. Perceptions of recovery and hunger were measured the next morning using visual analogue scales. Results: There was a significant difference $(p=.044)$ between the PRO $(M=$ 7.52; $\mathrm{SD}=1.86)$ and PLA $(M=5.27 ; \mathrm{SD}=2.01)$ group in terms of recovery with the PRO group reporting better recovery. No group $x$ time interactions were observed for CK, CRP or muscle performance. Conclusion: Casein supplementation 30 minutes prior to sleep enhances the perception of recovery the next morning from a bout of strenuous resistance training in athletes. 


\section{An investigation into the cognitive dynamics of batting: measurement of information processing in amateur cricket batsmen.}

Introduction: Batting in cricket is a demanding task, requiring physical and mental capabilities. While batting, players must offset a number of factors to ensure ultimate concentration and performance. While the bowler and the forthcoming delivery are the primary concern, external influences (crowd dynamics, field placement, information from the coaching staff and captain as well as previous performances) have a substantial affect on batting performance. In order to understand how batsmen cope with these factors, we must first understand the basic information processing capabilities required of batsmen when batting for extended periods. These have yet to be established, and as such form the basis of this investigation.

Methods: Fifteen top order (1-5) amateur batsmen from the Eastern Cape province of South Africa were recruited for this investigation (mean $\pm \mathrm{s}$ : age $17 \pm 0.92$ years; stature $1.75 \pm 0.07 \mathrm{~m}$; body mass $78.29 \pm 13.21 \mathrm{~kg}$ ). Participants completed a six-stage, 30-over batting simulation (BATEX@) interspersed with five periods (pre, post 5, 15, 25 and 30 overs) of cognitive assessment (CogState brief test battery, Melbourne Australia). Physical performance was assessed throughout the simulation through sprint times.

Results: Sprint times slowed significantly $(P<0.05)$, with the slowest times observed in stage 6 . Repeated shuttle running during prolonged batting significantly $(\mathrm{P}<0.05)$ impaired high-order cognitive performance. Executive function during the Groton maze-learning task was significantly impaired only post batting. Further, task performance illustrated a speed accuracy trade-off where significantly $(P<0.05)$ faster processing speed came at the expense of higher $(\mathrm{P}<0.05)$ error rates. In low order-tasks speed of processing and accuracy responses were unchanged.

Conclusion: Duration of an innings is a key determinant of batting performance. Further, under ideal laboratory conditions where external batting influences are reduced, it is evident that cognitive responses of batsmen deteriorate over time. Therefore, future research should investigate how mitigating factors affect cognitive performance and how interventions can be introduced to improve accuracy while maintaining processing speed. Further, coaches should utilise prolonged batting spells and shorter duration innings' in conjunction with shuttle running in preparation for matches. This combination may facilitate coping strategies that can be implemented during match play.

Janine Gray

Affiliation: CSA research consultant

Additional Authors: Janine Gray, Wayne Derman, Christopher L Vaughan, Paul W Hodges

\section{What is the role for the local muscles in protecting the spine during fast bowling?}

Introduction: The vertical ground reaction forces associated with fast bowling are between 4 and 7 times a bowler's body weight. The bowling reaction is a complex movement involving movement of the lumbar spine in all 3 planes of movement, very often at the extreme of range of movement. Lumbar shear forces during bowl have been found to be significantly greater in bowling compared to treadmill running. The role of the deep stabilisers in counteracting these large forces is not clear.

Objective: To investigate the function of the local muscles in bowlers with and without lower back pain in cricket fast bowlers.

Methods: The function of the deep stabilisers (Transversus abdominus-TrA; multifidus) was assessed using electromyography in 6 adolescent fast bowlers with low back pain (LBP) and 6 fast bowlers without during 3 different tasks with decreasing stability and increasing complexity. These included a supine rotation task, a rapid limb task and fast bowling.

Results: During the rotation task the TrA displayed an asymmetrical activation pattern. There was less TrA and multifidus activity in patients with pain. The normal anticipatory pattern which was evident in fast bowlers without LBP was delayed in fast bowlers with LBP during the rapid limb task. In addition, the TrA activity was again asymmetrical in both groups of bowlers. During bowling the TrA on the non-dominant side exceeded $70 \%$ of the peak activity between BFI and FFI for a longer duration in bowlers with TrA. However, the function of the TrA on the dominant side was less before and after foot contacts in bowlers with LBP.

Conclusion: The TrA appears to play a role in stability during rotation which is particularly relevant in sports like cricket. Crickets with LBP had comparatively less activity of both multifidus and TrA which demonstrates a dysfunction in the lumbar spine stability system.

\section{Tania Gregory}

Affiliation: Stellenbosch University

Additional Authors: Tania Gregory, Karen Estelle Welman

\section{Somatosensory training improves mobility and fear of falling in individuals with mild to moderate Parkinson's disease}

Introduction: Recent research has indicated that deficits in proprioception have a negative affect on postural control (PC), but that the precise contribution to postural instability in Parkinson's disease (PD) remains unclear (Bekkers et al., 2014). The study investigated whether an 8-week somatosensory training program (SSTP) will influence mobility and fear of falling in individuals with PD.

Methods: Thirty-seven individuals with idiopathic PD $(67 \pm 9$ years; Hoehn \& Yahr: $2 \pm 1)$ were divided into either a somatosensory training group (EXP; $n=24$ ) and placebo group (PBO; $n=13$ ). The Movement Disorder Society - Unified Parkinson's Disease Rating Scale (MDS-UPDRS), TimedUp-and-Go (TUG), as well as Fall Efficacy Scale-International (FES-I) were assessed before and after the 8 weeks.

Results: A treatment effect was found in the TUG $(p=0.0001)$, part III $(p=0.02)$, total score of MDS-UPDRS $(p=0.02)$ as well as in FES-I $(p=0.02)$ for EXP. The EXP group improved in the TUG after the intervention $(p<0.001)$ and showed strong tendency for improvement in part III of MDS-UPDRS post-intervention $(p=0.05)$. Additionally, a significant group difference was found after the intervention for the TUG $(p=0.01)$, with EXP showing improved results compared to PBO in both instances.

Conclusions: The positive findings of this study provide evidence that this SSTP can improve mobility and fear of falling in PD individuals. 


\section{Video Analysis of Concussion Injury Mechanism in Under-18 Rugby - a preliminary study}

Introduction: Understanding the mechanism of injury is necessary for the development of effective injury prevention strategies. Video analysis of injuries provides valuable information on the playing situation and athlete-movement patterns, which can be used to formulate these strategies. The aim of this study was to conduct video analysis of the injury mechanisms of concussion in Under-18 Rugby.

Methods: Injury reports for 18 concussion events were collected from the 2011-2013 under-18 Craven Week tournaments and video footage was recorded for all three years. Based on the injury events, a representative 'control' sample of matched non-injury events in the same players were identified. The video was then retrospectively analysed. Ten injury events ( 5 tackle, 4 ruck, 1 aerial collision) and 83 non-injury events were analysed. Results: Sixty percent of players were unaware of impending contact. For the measurement of head position upon contact, $43 \%$ had a 'down' position, $29 \%$ the 'up and forward' and $29 \%$ the 'away' position $(n=7)$. The speed of injured tackler was observed as 'slow' in $60 \%$ of injurious tackles ( $n=5)$. In three of the four rucks in which injury occurred $(75 \%)$, the concussed player was acting defensively either in the capacity of 'support' ( $\mathrm{n}=2)$ or as the 'jackal' ( $n=1)$.

Conclusion: Interventions aimed at improving peripheral vision, strengthening of the cervical muscles, targeted conditioning programmes to reduce the effects of fatigue, and emphasising safe and effective playing techniques have the potential to reduce the risk of sustaining a concussion.

\section{Erik Hohmann}

Affiliation: Musculoskeletal Research Unit, CQ University, Australia Medical School University of Queensland,

Additional Authors: Erik Hohmann, Tony Young, Ross Clark, Adam Bryant, Peter Reaburn

\section{Using the Nintendo Wii to measure balance as a predictor of functional outcome in ACL reconstructed knees - a novel technique}

Introduction: The purpose of this project was to assess the relationship between single leg standing balance using the Nintendo ${ }$ Wii ${ }^{T M}$ Balance Board (NWBB), functional outcome measured by the hopping tests, muscle strength and the IKDC score in subjects with ACL- reconstructed (ACLR) knees.

Methods: 25 ACL reconstructed patients with a mean age of $29.32+9.40$ years (range 17 years to 53 years) and a mean of $40.4+52.14$ months since their respective operations were included. The single leg standing balance was tested using the Nintendo Wii (NWBB) and Centre of Pressure (COP) pathlengths was recorded for thirty seconds in both the reconstructed and contra-lateral leg. The NWBB was also used to measure isometric strength of both the quadriceps and hamstring muscle groups. In addition all subjects performed a single- legged hopping, vertical jumping test and the IKDC scoring system was completed.

Results: Statistical analysis using a linear least square regression model demonstrated that standing balance is a strong and significant predictor $(p=0.03)$ of functional outcome.

Conclusion: The results of the current project clearly demonstrated a significant relationship between standing balance and functional outcome. The results highlight the need to also focus on proprioceptive rehabilitation on patients following ACL reconstruction.

\section{Erik Hohmann}

Affiliation: Musculoskeletal Research Unit, CQ University, Australia Medical School University of Queensland,

Additional Authors: Erik Hohmann, Elisabeth Livingstone, Adam Bryant, Kevin Tetsworth, Andreas Imhoff

\section{Tibial acceleration profiles in young female athletes during the menstrual cycle as an indicator for dynamic stability.}

Purpose: It is widely accepted that musculoskeletal injuries in sports are sports specific and not sex specific and raises the question as to why female athletes have a higher incidence of knee injuries in particular ruptures of the anterior cruciate ligament. Tibial acceleration is one of the main indicators of dynamic stability and tibial shock attenuation. It has been shown that athletes who are able to arrest tibial acceleration faster tend to display greater knee functionality whether ACL deficient or reconstructed. The purpose of this study is to investigate tibial acceleration profiles in young female athletes during the different stages of the menstrual cycle.

Methods: Eleven females aged 16-18 years participated in this study and were compared to a male control group. Female subjects were tested at each of the four phases of the menstrual cycle: menses, follicular, ovulation and luteal. On each test occasion, acceleration transients at the proximal tibia were measured whilst subjects performed an abrupt deceleration task (simulated netball landing). The male control group was recruited from the local development rugby team.

Results: No significant differences were found between the different phases of the menstrual cycle for peak tibial acceleration (PTA; $P=0.57)$, and time to zero tibial acceleration (TZTA; $\mathrm{P}=0.59$ ). However, there was a significant difference for time to peak tibial acceleration (TPTA) between menstruation and follicular $(P=0.04)$, menstruation and ovulation $(P=0.001)$, menstruation and luteal phase $(p=0.002)$, and follicular phase and ovulation $(P=0.007)$. In the male control group, no significant between test session differences were observed for $P T A(P=0.48)$, TZTA $(P=0.08)$ and TPTA $(P=0.29)$. While there were no significant between group differences for PTA $(P=0.21)$ and TZTA $(P=0.48)$, significant between-group differences were observed for TPTA $(P=0.001)$.

Conclusion: The results of this project strongly suggest that serum estrogen fluctuations have an effect on tibial acceleration profiles in young female athletes during different phases of the menstrual cycle. 


\section{Does muscle strength influence knee functionality in the ACL-deficient and ACL-reconstructed knee?}

Purpose: Functionality in the Anterior Cruciate Ligament (ACL)-deficient and ACL-reconstructed knee is multi-factorial and no single biomechanical variable is determinant of knee functionality. The purpose of this study was to investigate the relationship between quadriceps and hamstring muscle strength and knee functionality in the ACL-deficient and ACL-reconstructed knee.

Methods: Forty four ACL-deficient patients with a mean age of 26.6 years were tested within 3 months of injury and 24 ACL- reconstructed patients with a mean age of 27.2 years were tested at 12 months after surgery. All reconstructed patients underwent surgical reconstruction within six months after ACL injury using bone-patellar tendon and interference screws. The Cincinnati Knee Rating System was used to assess knee functionality. Muscle strength was assessed with the BiodexTM Dynamometer. Isokinetic concentric and eccentric peak torque ( $\mathrm{Nm} / \mathrm{kg})$ of the hamstring and quadriceps muscle were tested at three different speeds: $60 \mathrm{deg} / \mathrm{sec}, 120 \mathrm{deg} / \mathrm{sec}$ and $180 \mathrm{deg} / \mathrm{sec}$. Isometric strength was tested in 30 and 60 degrees of knee flexion. Both the involved and non-involved legs were tested to calculate symmetry indices.

Results: The mean Cincinnati score in the ACL-deficient subject was $62.0 \pm 14.5$ (range 36-84). The mean Cincinnati score in the ACL-reconstructed knee was $89.3 \pm 9.5$ (range 61-100). Significant relationships between knee functionality and muscle strength in the ACL-deficient group were observed for knee symmetry indices $(r=0.38-0.50, p=0.0001-0.05)$. In the ACL-reconstructed group significant relationships between knee functionality were observed for isometric and isokinetic peak torque of the involved limb $(r=0.46-0.71, p=0.0001-0.007)$.

Conclusion: The findings of this study strongly suggest that neither peak quadriceps or hamstring torque were correlated of knee functionality in the ACL-deficient knee. However, leg symmetry indices were correlated to knee functionality. In the ACL-reconstructed, knee symmetry indices were not related to knee functionality but peak quadriceps and hamstring isokinetic concentric and isometric were.

\section{Erik Hohmann}

Affiliation: Musculoskeletal Research Unit, CQ University, Australia Medical School University of Queensland, Australia Additional Athors: Erik Hohmann, Kevin Tetsworth

\section{Glenoid Version and Inclination is a risk factor for anterior shoulder dislocation}

Introduction: Whilst the contribution of both the capsule-ligamenteous structures and dynamic muscle balance to shoulder stability has been well documented, the role of the osseus anatomy of the glenoid has not been well established. The purpose of this study was to investigate glenoid version and inclination in patients with a documented anterior shoulder dislocation compared to a control group.

Methods: All patients presenting to the knee and shoulder clinic below the age of 50 years who underwent MRI for a shoulder condition were included. Version was measured on axial and inclination on T2-weighted spin-echo axial coronal images. The MR images of 66 patients with a mean age of $24.6+8.6$ years with a confirmed traumatic anterior shoulder dislocation (study group) and 129 patients with a mean age of $37.6+9.5$ years (control group) were evaluated.

Results: The mean retroversion in the study group was $3.7+4.4$ degrees and $6.1+5.2$ degrees in the control group. The difference between the two groups was significant $(p=0.001)$. The mean inclination in the study group was $2.0+6.6$ degrees sloping downwards and $2.5+7.2$ degrees sloping upwards in the control group. The difference between the two groups was also significant $(p=0.0001)$.

Conclusion: The results of this project strongly suggest that both osseous version and inclination are a risk factor for anterior shoulder dislocation.

Louis Holtzhausen

Affiliation: Division Sport and Exercise Medicine, University of the Free State

Additional Authors: Johan Myburgh, Louis Holtzhausen, Gert van Zyl

\section{Exercise Prescription by South African Doctors Part 2: Knowledge, Attitudes And Self-Perceived Competence Towards Exercise Prescription Among Final Year Medical Students}

Introduction and aim: Physical activity (PA) promotion is an integral part of preventative health care and reducing morbidity and premature mortality. Many barriers exist in implementing exercise prescription into the health care paradigm, possibly including inadequate undergraduate medical training. The aim of the study was to investigate the knowledge, attitudes, and self-perceived competence towards exercise prescription, as well as own exercise habits, amongst final year medical students from two South African universities .

Methods: A descriptive, cross-sectional study was conducted by means of an anonymous, self-reported questionnaire. Descriptive statistics was used, and correlations calculated at $95 \%$ confidence intervals.

Results: Only $16.9 \%$ of South African medical students obtain knowledge regarding exercise

prescription from their medical curriculum (24.7\% from University A and $9.6 \%$ from University B). Only $62 \%$ of students achieved an overall pass mark on the fundamental principles of exercise prescription. The vast majority (97.9\%) of University B students do not know formal guidelines for exercise prescription and $35.1 \%$ of them are unlikely to prescribe exercise $(p=0.0001)$, compared to $22.5 \%$ from University A. University B students feel more competent in prescribing exercise correctly than University A (23.6\% vs. $15.2 \%)$. Formal guidelines for exercise prescription is regarded as highly important by $57.8 \%$ of students, but only $19.3 \%$ feel highly competent in using it to prescribe exercise. A direct correlation exists between students' health habits and attitudes toward preventive counselling. Only $17 \%$ of students meet the ASCM's criteria for exercise for health.

Conclusions: The knowledge of South African final year medical students on exercise prescription

is inadequate, rendering them unlikely to prescribe exercise. Minimal curricular input on exercise prescription at one university made a significant difference in knowledge and

attitudes. Review of curricular content is recommended to include principles of exercise prescription. The promotion of physical activity to medical students during their training years is also recommended, to contribute to their health and increased awareness of the benefits of regular exercise. 
David Lee

Affiliation: Griffith University, Griffith University Centre for Musculoskeletal Research, Menzies Health Institute Queensland

Additional Authors: David Lee, Rod Barrett, Richard Newsham-West, Michael Ryan

\section{Regional Strain Variations of the Human In-Vivo Patellar Tendon using Digital Image Correlation (DIC)}

Introduction: In-vitro research has shown that strain variations occur within and along the fibers of the patellar tendon. Consequently, it has been hypothesised that these variations may contribute to the occurrence of patellar tendinopathy and may explain the specific localised lesion that occurs with the pathology. The primary aim of this study was to apply DIC to investigate the regional strain distributions within the human in-vivo patellar tendon. Specifically this study assessed 1) variations in mean strain between the proximal, mid, and distal tendon regions; and 2) variations in mean strain between the deep and superficial layers of each region.

Methods: Seven adult subjects ( 5 males, 2 females; age $=30.5+/-3.5$ y) performed rate controlled voluntary ramped contractions to a normalised target torque over a specified rate with simultaneous collection of sagittal plane imaging of the patellar tendon and knee extensor torque using real time B-mode ultrasonography and dynamometry, respectively. Ultrasound images were subsequently processed using specialized software to estimate regional strain variations within the in-vivo patellar tendon.

Results: Mean strain along the full length of the patellar tendon was estimated at $4.68 \%( \pm 1.64 \%)$ with no significant difference between deep and superficial layers $(4.64 \%( \pm 1.68 \%)$ vs $4.7 \%( \pm 1.66 \%))$. Strain estimates were found to be highest at the proximal and mid tendon regions when compared to the distal region $(7.27 \%( \pm 2.64 \%)$ vs $5.84 \%( \pm 2.83 \%)$ vs $-1.4( \pm 3.79 \%)$. Strain estimates for the deep tendon layer were found to be higher when compared to the superficial layer for the proximal and mid regions $(7.8 \%( \pm 2.81)$ vs $6.73 \%( \pm 2.58 \%)$ and $6.49 \%( \pm 3.14 \%)$ vs $5.15 \%( \pm$ $2.61 \%)$. For the distal region, the superficial layer estimates were found to be higher than the posterior layer $(1.41 \%( \pm 3.03 \%)$ vs $-4.07 \%( \pm 4.89))$.

Conclusion: This study shows significant regional strain variations exist within the patellar tendon during ramped isometric contractions. Higher strain in the proximal deep tendon layer would suggest stress overload as an etiological factor in patellar insertional tendinopathy. Strain estimates for the distal deep layer suggest that this region undergoes compression during isometric knees extension.

James Leitao

Affiliation: Spine Unit and Pain Management Centre

\section{Return to Sport: A Spine Surgeon's perspective}

Introduction: Although there are isolated protocols, there are currently no clear guidelines for sports therapists following spine injury or spinal surgical interventions. Both elite and regular athletes are unsure about what to expect after a back injury or spine surgery. All are keen to return to sport as soon as possible. There needs to be sensible and standardized approach to guide and rehabilitate individuals with back injury or spine surgery. The goal of any rehabilitation should be individualised depending on the diagnosis, surgical intervention, pain, time since surgery and specific sport.

When to return to sport, which type of sport and the role of prehabilitation are some key questions that need to be addressed with the individual athlete. Avoiding the disruption of the healing process by preventing excessive loading of the spine in all planes, in the early stages of repair, must be the goal.

Based on biomechanical aspects of the spinal conditions with which the athlete presents, spine surgeons normally classify spinal disorders. Athletes with discogenic pain have varying lengths of recovery period and can return after a short interval of rest and rehabilitation. The risk of recurrence is low. Athletes with large disc herniation's and pain should be managed conservatively initially. The risk of recurrence is higher with a broad based uncontained disc herniation. Athletes with surgical intervention should have a tailored approach for return to sport. The approach should be based on structural integrity of the spine and will vary from a few weeks for endoscopic surgery to a few months for Spinal fusion.

Summary: A Multidisciplinary approach to return to sport following spine injury or surgical intervention along with graduated return to sport should be the key focus. Avoiding excessive loading in all planes of motion in the early stages of healing and a significant prehabilitation is essential.

Physiotherapists, Strength and Conditioning

specialist, Biokinetist and sports technical coach have to play a role in an integrated manner.

Surgeons must share information about the surgical procedure and the stability of the spine with the patient and all therapists. 


\section{Rehabilitation of a SA Rowing Athlete following serious illness}

Background: The 24-year-old athlete began rowing at school in 2003 and joined the Elite squad in 2008. He achieved Gold and Silver medal status at U23 World Championships in 2 consecutive years.

History: Prior to diagnosis he presented with an 18 - 24 month history of recurrent upper respiratory tupper respiratory tract infections, occasional episodes of tiredness and fatigue and a slowly, progressive slight decrease in performance.

Clinical: The clinical examination was essentially normal.

Investigations: The routine bloods showed anaemia of chronic disorder and raised CRP and he was investigated for chronic infection during which he was treated for $\mathrm{H}$. pylori infection.

As things did not settle he was investigated further with a high resolution CT scan, bone marrow biopsy, lymph node biopsy and a PET scan.

He was diagnosed with Hodgkins Lymphoma Stage IV with involvement of the bone marrow and spleen.

Treatment: He was treated with Escalated BEACOPP - Bleomycin, Etoposide, Adriamycin, Cyclophosphamide, Oncovin, Prednisone and Procarbazine. The regime was chosen for his disease status as well as the side effect profile, which had advantages for an athlete.

The PET scan was clear after 2 cycles and after completion of the full regime.

Complications during treatment

Pneumonia following bone marrow suppression

Return to training

Points of concern

1. Bone marrow suppression

2. Drug side effects

1. Bleomycin

2. Adriamycin

3. Prednisone

4. Weight gain

5. Reintegration into the programme considering the athlete had the mind of an athlete but the body of a post chemotherapy patient

Return to full training was graded. Restrictions were placed on his training workload and a low intensity of work was enforced with a heart rate cap and a reduced length of session.

Running was avoided until an optimum weight was achieved.

He was given mental support during this period, as there was a high level of frustration.

After 6 weeks of low-grade training he was fully assessed for signs of chemotherapy toxicity with a full physical examination, a full blood screen, an extensive cardiac assessment, which included an echocardiogram and stress ECG, as well as Lung Function Tests.

There is ongoing assessment for signs of disease relapse and although the risk 3\%), monitoring will be close for the next 5 years.

Outcome: He has returned to full training and is currently working towards the World Championships

James MacDonald

Affiliation: Nationwide Children's Hospital, Columbus, OH USA

Additional Authors: James MacDonald, Mitchell Selhorst, Anastasia Fischer, Reno Ravindran, Richard Rodenberg, Eric Peters, Kristine Graft, Eric Welder

\section{Prolonged Rest Versus Early Physical Therapy in Patients with Active Spondylolysis}

Introduction: Spondylolysis is a stress fracture most commonly occurring in the lumbar spine. This injury is common in adolescent athletes, accounting for as much as $40 \%$ of all low back pain in this population. Currently, there is no evidence to guide physicians on when to make a safe referral to physical therapy [PT] for these patients. There were two primary objectives of this study: (1) to determine if the amount of time prior to referral to PT affects the time to make a full return to activity for patients with an acute spondylolysis (2) To assess the safety of an early referral to PT compared with a longer rest period prior to PT in patients with an acute spondylolysis.

Methods: This was a retrospective study which consisted of the review of each patient's medical chart and lumbar imaging.

Participants: One hundred and ninety-six patients with an acute spondylolysis injury met the inclusion criteria during the pre-selected review period. Patients were sub-grouped into 2 groups by physician referral patterns: an aggressive referral to PT group $(<10$ weeks) and a conservative referral to PT group (> 10 weeks). Safety was assessed by calculating the number of adverse reactions experienced in each group during the course of treatment. Data Analysis: Mann-Whitney $U$ tests were used to analyze the first study objective. Relative risk of an adverse reaction was calculated to assess the second study objective.

Results: Median days to a full return to sport for aggressive PT referral group (115.5 days IQR 50.5) and conservative PT referral (140.0 days IQR 40.3) was significantly different $(p=0.003)$. A total of 11 patients had documented adverse reactions during the course of treatment for acute spondylolysis. Although there was a higher percentage of patients who experienced an adverse reaction in the conservative referral to PT group, this difference was not statistically significant $(p=0.509)$.

Conclusions: The results of this retrospective study show that patients of physicians who referred to physical therapy sooner returned to sport more quickly without an increased risk of adverse reaction 


\section{Management Dilemmas in Treating Cricket Injuries}

Introduction: Injuries in cricket players are well documented as to which areas of the body that are injured, what type of injury is more common and which activity or what type of player sustains them. However many injuries that prove to be diagnostic challenges, or are not often seen and thus missed. Additionally, there are injuries whose management may be affected by the state of the game, the stage of a series or the role of the player. This paper presents a series of challenging cases to highlight difficult management decisions that need to be taken.

Methods: Cases that were diagnostic dilemmas and management challenges in West Indies cricket were selected from the injuries treated by the author

These include:

A rare shoulder injury, which provided diagnostic challenges

A head injury was a management challenge

Finger injuries which were interesting and could have been managed in different ways

Knee injury which had negative MRI findings but required surgery.

The format of the presentation is preferably interactive, in which case histories will be presented and the audience will be asked to participate in discussion before the final management is revealed.

Results: The results of each case will be discussed with the audience aiming to focus on the cases in particular, but give an insight into management decisions that are often required in elite cricket.

Conclusion: This paper will highlight injuries that are either common in cricket but pose difficult management considerations and could have many different ways of treating. It will also present some rare injuries that are often missed.

Akshai Mansingh

Affiliation: University of the West Indies

Additional Authors: Akshai Mansingh, Christopher Clark, Isabel Moore, Craig Ranson,

\section{Changing Trends of Injuries in International Cricket; Reviewing 10 years of Injuries in West Indies Cricket}

Introduction: Only Australia and West Indies have reported a decade of longitudinal injury data. During this time there have been variations in the types of injuries seen, as well as time lost due to injury, prevalence and incidence of injury.

This paper looks at injuries sustained in West Indies Cricket over ten years and identifies changing trends and how these have affected the game.

Methods: Prospective injury surveillance of players selected for the West Indies Cricket Team from 2005 to 2014 was collected based on consensus methods. Additionally both time-loss (TL) injuries (player unavailable for match selection) and non time-loss (NTL) injuries (requiring medical attention but player available for selection) were included.

Results: There were 404 injuries [212 NTL injuries; 192 TL injuries] by 79 players, at 1.21 injuries/100 days (0.63 NTL; 0.57 TL). Incidence of NTL injuries was higher than TL injuries during training ( 0.28 vs. 022/100 days), but similar during matches (0.29/100 days). More NTL injuries ( $32 \%$ ) were chronic compared to TL injuries (15\%). Mild injuries ( $<3$ days lost) had the highest incidence $(0.23 / 100$ days). Fielding resulted in the most injuries. The hand, lower back, shoulder and knee accounted for $42 \%$ of injuries.

There were more acute injuries sustained but required less time lost from the game. Trends changed from chronic injuries requiring long periods out of the game (stress fractures of the back) a decade ago, to soft tissue strains with less than a week out of the game now.

Conclusion: There was a low injury incidence over a decade, but a high proportion of TL injuries compared to other studies. Fielding resulted in the most injuries, which may require attention to technique. Less time is lost from play due to the change in types of injuries, better medical support and quicker detection of injury. As most NTL injuries took place during training, modifications in training methods may need to be considered.

Prevention strategies for hamstring strain injuries should be implemented focusing on known risk factors, such as muscle strength deficiencies, whereas for ankle injuries, being able to manage on-going NTL injuries should be targeted.

RobRoy Martin

Affiliation: Duquesne University

Additional Authors: RobRoy Martin, Ben Kivlan, Hal Martin

\section{A Cadaveric Study of the Dynamic Internal Rotation Impingement (DIRI) and Dynamic External Rotation Impingement (DEXRI) Tests}

Purpose: Labral pathology of the hip joint is commonly associated with an abnormal abutment of the femoral neck to the acetabular labrum. There have been many clinical tests developed to help determine the presence of labral pathology with limited evidence of validity to support their use. The Dynamic Internal Rotation Impingement (DIRI) and Dynamic External Rotation Impingement (DEXRI) tests were proposed by Martin to comprehensively assess all zones of the labrum for potential pathology due to femoroacetabular impingement. The purpose of this study was to assess the validity of the DIRI and DEXRI tests by describing the point of contact of the femoral neck relative to specific zones of the acetabular labrum in a cadaveric study.

Subjects: 26 hips from 14 embalmed cadavers ( 7 male; 7 female) with a lifespan ranging between 51-95 years were used for this study.

Methods: The pelvic region of each cadaver was skeletonized and the labrum marked into 5 geographic zones according to llizaliturri as follows: Zone 1 - anterior-inferior, Zone 2 - anterior-superior, Zone 3 - superior, Zone 4 - posterior-superior, Zone 5 - posterior-inferior. From a neutral anatomical position the DIRI test was performed by moving the femur through a full arc of flexion, adduction and internal rotation while maintaining contact of the femoral neck to the labrum. From position of maximum flexion the DEXRI was performed by moving the femur through a full arc of extension, abduction, and external rotation while maintaining contact of the femoral neck to the labrum. When the femoral neck made contact with each of the 5 labral zones the position of hip flexion was recorded to describe the arc of motion in the sagittal plane in which the DIRI and DEXRI tests were in contact with each specific zone of the labrum.

Results: The DIRI test made contact to zones 1-3. Contact with the anterior-inferior labrum (Zone 1) occurred in less than $57^{\circ}$ of hip flexion, while contact with the anterior-superior labrum (Zone 2) occurred between $57^{\circ}-101^{\circ}$. Zone 3 was contacted in a maximally flexed position. The DEXRI test made contact to zones 3-5. Contact with the posterior-superior portion (Zone 4 ) of the labrum occurred between $106^{\circ}-15^{\circ}$ flexion. The posteriorinferior portion (Zone 5) of the labrum contacted the femoral neck as the hip joint was moved towards midline through an arc of $15-47^{\circ}$ flexion .

Conclusion: The DIRI and DEXRI tests may be used to discriminate the location of labral pathology from anterior-inferior (Zone 1) to posteriorinferior (Zone 5) positions.

Clinical Relevance: Noting the position of hip flexion in which pain reproduction occurs during the DIRI and DEXRI tests may help to identify the specific zone(s) of labral pathology. 


\section{The Functional Movement Screen in the Prediction of Injury of High School Cricket Pace Bowlers}

Introduction: To ensure young cricketers remain injury free all fitness and medical staff should identify risk factors and develop preventative strategies. The Functional Movement Screen (FMS) has been developed as a pre-participation screening tool to assess the quality of an athlete's fundamental movement patterns, identify asymmetries and limitations and establish the risk of sustaining an injury. A score of 14 or less have been found to predict injury among various sport groups. No documented research confirming the predictive validity of the FMS among cricketers could be found.

Objective: To determine if pre-season FMS score is a valid predictor of inseason injury among adolescent pace bowlers.

Methods: This is a prospective observational quantitative study. Male adolescent pace bowlers $(n=27)$ who were injury free at the start of the season. Bowlers performed the seven FMS movements and were scored according to the scoring criteria as set out by the authors of the FMS. Injury incidence were monitored by bowlers completing the standardised self-administered questionnaires pre-season, in-season (monthly) and postseason. The independent student t-test and Fisher's exact tests were used to compare the FMS scores of the injured and non-injured bowlers as well as the injured and non-injured bowlers that scored $\leq 14$ with significance set at $p \leq 0.05$.

Results: The mean FMS score for the sample was $16.44( \pm 2.41)$. The non-injured group $(16.55 \pm 2.57)$ score slightly higher than the injured $(16.1 \pm 2.07)$ group but there was no significant difference between the groups. There was also no significant difference between injured and noninjured bowlers who scored $\leq 14$. A total FMS score of 14 does not provide the sensitivity $(0.2)$ needed to assess injury risk among adolescent pace bowlers and no other accurate cut-off score could be calculated.

Conclusion: Pre-season observed total FMS score is a poor predictor of in-season injury among adolescent pace bowlers. More research should be conducted to determine if a specific FMS test or asymmetry is a more valid predictor of injury.

\section{Crosby Mulungwa}

Affiliation: Division Sport and Exercise Medicine, University of the Free State

Additional Authors: Crosby Mulungwa, Louis Holtzhausen

\section{The Use of Traditional Medicine and Rituals in Professional Soccer in South Africa}

Introduction and aim: Traditional medicine(TM) and Traditional rituals (TR) are anecdotally widely

used in soccer in South Africa. There is no scientific information on content of substances, efficacy, possible adverse effects, or anti-doping status of TM and TR in sport in South Africa. The aim of the study was to investigate the types, indications and beliefs regarding TM and TR in South African professional soccer as baseline data for further investigation (Ethics clearance ECUFS 56/2012).

Methodology: A descriptive study was conducted, with qualitative and quantitative components.

The study population was selected using purposive sampling because of the rarity of people willing to divulge information on this topic. Five former South African professional soccer players were interviewed using a semi-structured interview guide.

Descriptive data was extracted, categorised and tabulated.

Results: The use of TM and TR was confirmed among South African professional soccer players. A list of TM and TR was identified. The main indications for TM and TR use are injury and illness management, performance enhancement and protection. The perceived efficacies of TM versus western medicine and of TM or TR for team success are equivocal. Secrecy about the use of TM and TR complicated research on this topic.

Conclusion: The common use, types, their indications and beliefs about TM and TR in South African professional soccer were recorded for the first time. An attempt was made to contextualise TM and TR use in soccer. There is insufficient scientific evidence or knowledge on efficacy, safety and legality of TM and TR in South Africa for healthcare workers to recommend it to athletes. Further scientific and socio-cultural investigation is strongly recommended.

Takalani Clearance Muluvhu

Affiliation: HPCSAAND BASA

Additional Authors: Takalani Clearance Muluvhu, MakamaAndries Monyeki, Meriam Mohlala, Gert Strydom

\section{Physical activity and selected health risk factors profiles among local government employees in Vhembe District, Limpopo Province of} South Africa.

Background: Research studies identify physical inactivity as a global health concern associated with non-communicable diseases of lifestyle affecting people from different walks of life. The purpose of this study was two-fold: to determine the prevalence of physical activity and risk factors of chronic diseases among local government employees in the Vhembe district, and to investigate the relationship between physical activity and risk factors of chronic diseases in the above-mentioned population.

Methods: A cross-sectional study design on an available sample of 540 (Men=253 and Women=287) local government employees in the Vhembe district in Limpopo province participated in this study. A standardised physical activity questionnaire was used to determine the physical activity index (PAI) of the participants; and clinical measures of body mass index (BMI), waist circumference (WC) and blood pressure (BP) were assessed.

Results: The results show that $53 \%$ of the employees do not participate in physical activity, with a higher percentage in women (49\%) compared to men $(47 \%)$. Additionally the results show the presence of both overweight and obesity $(21 \%$ and $44 \%)$, with women ( $23 \%)$ accounted for a higher percentage compared to the men (21\%). Finally, the results showed that physical inactivity was positively associated with all measures of BMI, WC and blood pressure (SBP and DBP), with a significant ( $\mathrm{p} \leq 0.05$ ) association with BMI.

Conclusions: It can be concluded that $53 \%$ of the 540 employees do not participate in physical activity and had a high percentage of obesity, especially the women. The physical activity index correlated with all measures of body mass index, waist circumference, and blood pressure, and was significantly associated with body mass index.

Keywords: Physical activity, health risk, local government employees, overweight 
Natalia Neophytou

Affiliation: Centre for Exercise Science and Sports Medicine, School of Therapeutic Sciences, Faculty of Health Sciences, University of the Witwatersrand, Johannesburg, South Africa

Additional Authors: Natalia Neophytou, Kerith D. Aginsky, Caitlin Tracey

\section{Posture and Isokinetic Shoulder Strength in Female, Water Polo Players}

Background: Water polo players are overhead athletes, presenting with shoulder muscular imbalances between the internal rotators (IR) and external rotators (ER) which may lead to changes in posture and increase predisposition to injury.

Objective: To assess posture and isokinetic shoulder strength of female, club level, water polo players.

Methods: A descriptive study assessing posture and isokinetic strength of the IR and ER shoulder muscles in 15 female, club level, South African water polo players (age: $21.3 \pm 1.5$ years). Posture was assessed using a posture grid. Isokinetic shoulder rotator muscle strength was tested over 5 repetitions concentrically and eccentrically at $60 \% \mathrm{sec}$ using a Biodex isokinetic dynamometer system 3 . The bilateral, reciprocal and functional $(\mathrm{DCR})$ ratios were calculated.

Results: Typical posture included a forward head, rounded shoulder, thoracic kyphosis, an elevated non-dominant shoulder, mild scapula winging, lumbar lordosis and an anterior pelvic tilt. The mean concentric reciprocal ratios for the dominant $(52.2 \pm 7.1 \%)$ and non-dominant $(51.9 \pm 6.5 \%)$ sides indicated some ER muscle weakness. However, the mean concentric (IR: $8.8 \pm 10.7 \%$ and ER: $10.4 \pm 6.3 \%$ ) and eccentric (IR: $6.1 \pm 10.4 \%$ and ER: $4.8 \pm 30.3 \%$ ) bilateral ratios were within normal limits as were the eccentric reciprocal ratios (D: $69.6 \pm 16.1 \%$ and ND: $67.3 \pm 10.9 \%$ ) and the DCR values (D: $0.75 \pm 0.2$ and ND: $0.75 \pm 0.1$ ).

Conclusion: There is a trend for these female water polo players to have rounded shoulders and forward head postures as well as ER muscle strength weakness, this combination could predispose the athletes to shoulder injury.

Kim Nolte

Affiliation: University of Pretoria

Additional Authors: Kim Nolte, Sasha Burgoyne, Heinrich Nolte, Julia van der Meulen, Lizelle Fletcher

The effectiveness of a range of sports bras in reducing breast displacement during incremental treadmill running and two-step star jumping

Introduction: The primary aim of the study was to investigate the effectiveness of a range of sports bras in reducing multi-planar breast displacement during incremental treadmill running and a two-step star jump. A secondary aim was to assess the relationship between perceptual bra comfort, bra fit and breast pain and measured breast displacement.

Methods: Seventeen females (mean age 22 years, range 18 - 31 years) with bra cup sizes B to C volunteered to take part in the study. Participants were assessed in the biomechanics laboratory of Ergonomics Technologies on 20 - 24 August 2012). Three dimensional breast movements were determined for six randomly assigned breast support levels during incremental treadmill running and two-step star jumping. Participants completed a perceptual questionnaire rating bra comfort, bra fit and breast pain.

Results: Not all the bras significantly reduced resultant breast movement compared to a control condition. Perceptual ratings of bra fit and bra comfort were stronger and more reliable predictors of breast pain in our group of relatively small breasted participants.

Conclusion: Therefore it is apparent that resultant breast movement and the reduction of such movement is a multifaceted function of breast size, bra design and movement/activity type. The variance in bra performance at different treadmill running speeds highlights this fact and indicates that sports bras should be carefully selected to best suit the activity and breast size of the user.

Habib Noorbhai

Affiliation: Cape Peninsula University of Technology

Additional Authors: Habib Noorbhai, Timothy Noakes

\section{A qualitative and biomechanical analysis of backlift batting techniques among skilled, semi-skilled and unskilled cricket batsmen}

Introduction: One of the first principles of cricket batsmanship that is coached from a young age is to play with a straight bat. This requires the bat to be lifted directly back towards the stumps with the elbow "up", pointing in the direction of the ball's trajectory. Limited studies to date have examined whether top international skilled cricketers (SC) actually use this traditionally described technique. No study has yet examined whether there are differences in the backlift and batting techniques of semi-skilled cricketers (SSC) and unskilled cricketers (UC).

Methods: Accordingly, we performed a descriptive, observational study of the backlift technique adopted by 65 of the most successful SC of all time based on their career averages, strike rate and runs scored. The batsmen were divided into two groups depending on whether they played the game, before or after 1954 and which described this backlift technique as the ideal. This was due to the first MCC coaching manual produced in 1954. We also investigated two study samples of SSC (comprised of both adolescent $(n=30)$ and amateur $(n=10)$ cricketers) and UC (comprised of only the young Calypso cricketers $(n=40)$ ). Various types of deliveries were bowled to the participants utilising a bowling machine. Biomechanical and video analysis were performed on all three participant groups utilizing Kinovea. Classifiers were utilised to identify the type of batting technique employed by all groups of batsmen.

Results: Surprisingly, more than $70 \%$ of SC did not adopt the traditionally taught technique. Instead they adopted a more looped action in which the initial movement of the bat was in the direction of the slips, and in extreme cases it was either towards the gulley/point region or having the face of the bat directed towards the off-side. More than $70 \%$ of UC adopted the lateral backlift batting technique whereas more than $70 \%$ of SSC adopted the straight backlift batting technique. Players begin to start adopting either the lateral or straight backlift batting technique around the age of 11 years. Cricketers who have a lateral angle of the backlift of more than 50 o have a better chance of hitting the ball with better timing and power.

Conclusion and Coaching Implications: Most coaching instruction today allows the backlift to be taken to first or second slip or have the bat face towards the stumps but not in the direction of point nor as part of a looped action. This suggests that traditionally taught batting coaching techniques may hinder rather than enhance future cricketing performance. If such players are not coached, they automatically hit the ball using a lateral technique which indirectly suggests that early coaching emphasising traditional techniques could be disadvantageous to the young cricketer. Future research is required to evaluate the coaching methods of the backlift batting techniques taught by coaches at various levels in most ICC countries.

Key words: Cricket batting, backlift batting techniques, biomechanics, qualitative, skilled, semi-skilled, unskilled 
Sarah J. Moss

Affiliation: Physical Activity, Sport and Recreation Focus Area, North-West University, Potchefstroom School of Health and Sport Sciences, Faculty of Science, Health, Education and Engineering, University of the Sunshine Coast, Australia

Additional Authors: Sarah J. Moss, S.O. Onagbiye, M. Cameron

Managing non-communicable diseases in an African Community: effects, compliance, and barriers to participation in a four-week exercise intervention.

Introduction: To determine the compliance, barriers, and effects of participation in a four-week exercise intervention to reduce risk factors for NCDs among Setswana-speaking community-dwelling Potchefstroom adults from a low resourced area of South Africa.

Methods: An exercise program and associated pre-post test were performed by 76 participants (men, $n=26$ and women, $n=50$ ) aged 35 to 65 years. Baseline and end tests included height, weight, hip and waist circumference, heart rate, blood pressure, glucose, cholesterol, quality of life, and cardiorespiratory fitness measurements. The intervention consisted of three days/week combined aerobic and resistance exercise at an intensity of $70 \% \mathrm{HRR}$ as determined at baseline. Compliance and barriers to participation were determined post-intervention by means of attendance registers and interviews. ANCOVA with adjustment for pre-test was performed for all repeated variables. The Cronbach's alpha coefficients for exercise benefits were 0.81 and for barriers 0.84 .

Results: Of the 26 men $(40.8 \pm 5.45$ years) and 50 women ( $43.6 \pm 7.8$ years) recruited, 54 completed the intervention $(71 \%$ compliance). The fourweek aerobic exercise intervention significantly reduced body mass, RPE and MCS in men, and body mass, BMI, VO2max, RPE, glucose, PCS and MCS in women. Participants reported that the exercise milieu as a major barrier to exercise compliance while the interviews reported lack of time.

Conclusion: A one month exercise intervention elucidated positive changes in risk factors for NCDs in a low-resource community. A drop-out rate of $29 \%$ in this study is consistent with other exercise intervention trials. Exploration of the reported barriers may be useful for planning to increase compliance with future programs.

Keywords: Physical activity intervention, cardiorespiratory fitness, quality of life, non-communicable diseases, adults, Setswana

Jessie Turner Pearson

Affiliation: Division of Exercise Science and Sports Medicine, University of Cape Town

Additional Authors: Jessie Turner Pearson, Lisa Micklesfield, Estelle Lambert

\section{A formative assessment of physical activity levels in pregnant women presenting at two public health clinics in Cape Town, South Africa}

Background: It has been proposed that physical activity pre-pregnancy, during pregnancy and post-partum reduces the risk of both the mother and the child developing obesity and its co-morbidities. Little is known about the physical activity patterns of pregnant South African women, while it is known that overweight and obesity are prevalent amongst South African women. The aim of this study was to describe the physical activity and sedentary behaviour patterns of pregnant women presenting at two public health clinics in Cape Town, as well as the barriers and facilitators associated with physical activity during pregnancy.

Methods: Forty women between 18 and 35 years of age (mean: $27 \pm 4.8$ yrs) and 12 and 25 weeks gestation (mean: $17 \pm 3.5$ wks) were included. The Global Physical Activity Questionnaire (GPAQ) was used to obtain data on self-reported physical activity and information on socio-economic status, sedentary time, knowledge, attitudes and beliefs about exercise during pregnancy, and self-efficacy related to exercising while pregnant was obtained via questionnaire.

Results: The sample was divided into active $(n=26)$ and inactive $(n=14)$ groups according to GPAQ guidelines. The only significant sociodemographic difference was that a greater proportion of women who had attended tertiary education than the active group (30\% vs. $19 \%$; $p=0.015$ ). For the whole sample, majority of PA $(9 \%)$ was walking for transport with only $30 \%$ of the women reporting leisure time activity and $12.5 \%$ participating in vigorous PA. The inactive group spent significantly more time sitting than the active group $(13.0 \pm 3.1 \mathrm{vs} .9 .1 \pm 3.7 \mathrm{hrs} / \mathrm{day}, \mathrm{p}=0.004)$, and the total amount of sedentary time (including sitting watching T.V., using the computer, travelling, eating or reading, and sleeping during the day) was significantly greater for the inactive group than the active group ( $14.9 \pm 4.0 \mathrm{vs} .10 .3 \pm 4.2 \mathrm{hrs} /$ day, $\mathrm{p}=0.003)$. Barriers to physical activity included knee, back and abdominal pain (93\%), being too tired to exercise $(63 \%)$, not having time to exercise (55\%), living in an unsafe neighbourhood (40\%). Facilitators of exercise during pregnancy included someone to exercise with (88\%), a specialised exercise programme for pregnant women (88\%), somewhere to exercise $(45 \%)$ and access to information about why exercise is important $(45 \%)$. Walking was identified as the most popular mode of exercise $(75 \%)$, followed by an exercise programme at home $(60 \%)$, yoga $(50 \%)$ and exercises at the community hall $(48 \%)$, while aerobics was the least popular choice (35\%).

Conclusions: Although the majority of the women in this study were classified as active, our study showed low levels of leisure time activity and high levels of sedentary behaviour. Thus, it is apparent that there is a need for an intervention which will educate women on the benefits of physical activity for them and their baby not only during pregnancy, but also throughout their lifetime.

\section{Nivash Rugbeer}

Affiliation: Department of Sport, Rehabilitation and Dental Science, Tshwane University of Technology, Pretoria

Additional Authors: Nivash Rugbeer, Serela Ramklass, Johan van Heerden

\section{Effect of group exercise frequency on health related quality of life in older persons residing in institutionalised care facilities}

Background: Worldwide, the proportion of older persons aged 60 years and older is increasing. The elderly within institutionalised setting are often neglected, with the probability of disease and disability being highly prevalent. Increasing age and visceral fat coupled with a lack of structured exercise results in inflammatory and pro-inflammatory processes, contributing to the deterioration of physical and physiological functioning. Health Related Quality of Life (HRQoL) in the context of the elderly is defined as their functional status and independence in engaging in activities of daily living. Little is known about the effect of group based exercise frequency on HRQoL among the elderly residing in aged care homes within the eThekwini central business district (CBD). The study aimed to determine the effect of group exercises $2 X /$ week vs $3 X /$ week on health related quality of life of older persons residing in age care homes in the eThekwini CBD.

Methods: A quasi-experimental design was used to compare the effect of a 12 week group exercise programme on two groups of participantoups of participants using pre-test and post-test procedures. A total of 100 participants were selected from five aged care homes. Twenty participants from each of the five facilities were randomly selected through convenient sampling. Admittance to the group was based on the outcome of a medical assessment by a sports physician. From the 20 participants, ten participants were randomly assigned to Group A (experimental) and 10 in Group B (observed group). Group A exercise three times a week and group B exercise two times a week for 12 weeks. The Medical Outcomes Study 36 -Item Short-Form Health Survey (SF-36) was used pre and post exercise to determine effect of intervention on health related quality of life.

Results: Results showed a significant difference in social functioning (Mean Participant Difference $(M P D)=13.35, p=0.00)$, vitality $(M P D=7.55, p=$ $0.00)$ and mental health (MPD $=5.11, p=0.03)$ post training thrice a week. There was a significant difference in social function post training twice a week $(M P D=12.15, p=0.02)$. Improvements in mental component summary scale $(p=0.03)$ post training thrice $(M P D=6.67, p=0.00)$ and twice $(\mathrm{MPD}=3.68, \mathrm{p}=0.03$ ) a week was also noted.

Conclusion: Elevated vitality levels, mental and social health benefits can be obtained irrespective of group exercise frequency $2 X /$ week or $3 X /$ week. This may assist the elderly in preserving independence and health within long term care facilities. 


\section{The Efficacy of a Community Based Soccer-Specific Hamstring Intervention in Black Amateur South African Players}

Introduction: Hamstring injury prevalence in soccer remains a major concern. Research in this context has focused on European populations with little attention given to other ethnic groups. The compatibility and applicability of such research to the South African context may be minimal due to fundamental differences in physical characteristics, as well as complexities with regards to implementation. The aim of this investigation was therefore twofold. 1) Identify the lower extremity strength profile of Black Amateur South African Players, and 2) Assess the efficacy of a community based intervention through the use of the Nordic Hamstring Exercise.

Methods: 19 Male Black Eastern Cape amateur players participated in a 12 week RCT ( $9=$ Control, $10=$ Intervention). All participants completed regular training, while the intervention group, in addition, performed the Nordic Hamstring exercise. The incremental intervention design was taken from Mjolsnes (2004). Concentric and eccentric isokinetic strength evaluations were performed pre and post intervention, for knee flexors and extensors, at speeds of 60 and $180^{\circ}$.s- 1.

Results: At $60^{\circ}$.s -1 and $180^{\circ}$.s -1 , Concentric Quadriceps Peak Torque (PT) showed no significant difference between the intervention and control groups ( $p=0.957$ and $p=0.3141)$. Eccentric Hamstring PT indicated a significant improvement at $60^{\circ} . s-1(p=0.004)$ in the intervention group, with no significant changes at $180^{\circ} . \mathrm{s}-1(\mathrm{p}=0.1477)$. With regards to the functional ratio (eccH:conQ), an interaction effect between group and time was found at $60^{\circ} . s-1(p=0.0148)$, while no changes were found between the control and intervention group at $180^{\circ} . s-1(p=0.495)$.

Conclusion: Findings indicate that the intervention was partially successful. While the intervention resulted in improvements at $60^{\circ} . \mathrm{s}-1$, these changes were not evident at the more functional velocity of $180^{\circ} . \mathrm{s}-1$. It can be concluded that Community based programs within SA have the potential to be effective; however, there are many barriers to implementation, including, language, ethnic and cultural differences, while a lack of resources and infrastructure play a significant role in a lack of development. More research of this nature is required to provide scientific support for structures and guidelines at an amateur level in SA, to ensure the efficacy of internationally successful interventions such as the Nordic exercise.

Marlene Schoeman

Affiliation: University of the Free State

Additional Authors: Louis Holtzhausen, Rudi de Wet, Marlene Schoeman

Clinical, Haematological and Biochemical Characteristics of South African Gold-Miners Presenting with Exercise-Associated Muscle Cramps, Part 2: Results and Discussion

Introduction: The pathogenesis of exercise-associated muscle cramps (EAMC) in mine-workers and other exercising populations is not clear. The aims of the study were to describe clinical, biochemical and haematological variables in gold miners with EAMC.

Methodology: A retrospective descriptive study of the data of underground mine workers who presented with EAMC over 18 months in a South African gold mine (CRA group) was compared with a control group of similar workers without EAMC from which data were collected before (CONPRE) and after (CONPOST) 8 hour shifts.

Results: There were 450 cases of EAMC in a population of 18430 mine workers over an 18-month period (2.5\%). Markers which were significantly different in the CRA group compared to the CONpost group were signs of dehydration (increased haematocrit and haemoglobin), muscle fatigue (elevated CK), muscle damage (elevated myoglobin), inflammation (elevated total white cell and lymphocyte count), elevated urea and creatinine, increased body temperature and lower fluid intake. The control group were well- to slightly over hydrated, with progressive muscle injury (increased CK levels, but no increase in myoglobin) and well maintained kidney function during a working week.

Conclusion: EAMC is associated with elevated inflammatory markers, dehydration and haemoconcentration, reduced absolute serum sodium and chloride levels, elevated creatinine and protein levels, and muscle damage (myoglobin). This study is the first to present a comprehensive profile of a population with EAMC, compared to a control group. The interpretation of these results should be done cautiously. Further investigation into the pathogenesis of EAMC will be guided by these results. Instead of compartmentalised theories, EAMC seems to occur in a collective set of systemic and local contributing factors.

Marlene Schoeman

Affiliation: Sport and Exercise Medicine, University of the Free State

Additional Authors: Marlene Schoeman, Ceri Diss, Siobhan Strike

\section{Jump landings in lower limb amputees: Mechanical considerations for prehabilitation}

Introduction: Jumping is a fundamental movement in many recreational sports and terminates in landing. Jump landing is a common cause for acute injury and osteoarthritis $(\mathrm{OA})$ which raise concerns for lower limb amputees who are encouraged to participate in recreational sport yet may be compromised to perform the jump landing safely due to the loss of the most distal joint and their predisposition to OA. The aims of this study were to investigate the loading experienced by transtibial amputees (TTAs) in vertical jump landings and explore compensatory mechanisms in controlling their downward momentum and attenuate the shock during landing to inform prehabilitation.

Methods: Six unilateral TTAs and ten able-bodied (AB) participants completed 10 maximal effort bilateral vertical jumps of which the highest jump was analysed. TTAs had to be at least 1 year post-amputation with no secondary pathology. The single jump with the greatest vertical position of the centre of mass in each situation was used for analysis. Data were collected using two Kistler force platforms synchronized with a 9-camera Vicon motion analysis system. AMann-Whitney $U$ test was used to assess bilateral differences and differences between the TTA and AB participants.

Results: Significantly larger $(p=0.041)$ impact forces $(25.25 \pm 4.89 \mathrm{~N} . \mathrm{kg}-1)$ were experienced by the TTAs on the intact compared to the prosthetic side. Similar impact forces to the AB participants were experienced despite landing from significantly lower $(p=0.000)$ jump heights. The TTAs performed a quasi-unilateral landing onto the intact limb, resulting mainly from the incapacity of the prosthetic ankle to plantarflex and active strategies to increase residual knee and hip flexion prior to touch-down. Loading rates onto the prosthetic side were significantly higher compared to both the intact side $(p=0.026)$ and AB participants $(p=0.000)$ due to reduced prosthetic ankle, knee and hip RoMs, resulting in reduced extensor moments and negative work. The TTA landing from the greatest height (similar to AB participants) experienced the smallest impact forces through a fairly symmetrical engagement of both limbs and going through large knee and hip RoMs.

Conclusion: Uninformed landing strategies employed by the TTAs pointed to avoidances to engage the prosthetic limb in the landing which exposed the intact limb to large impact forces which may lead to degenerative disease over the long term. Attempts to keep the residual knee joint erect resulted in inadequate RoMs over which to generate extensor moments and produce negative work to dissipate the landing shock. Landing with an extended knee evoked large loading rates which may increase acute injury risks and cause residual skin breakdown if repetitive jumping were to occur. The results imply that safer landing techniques can (and should) be taught during prehabilitation. 
Marlene Schoeman

Affiliation: Division Sport and Exercise Medicine, University of the Free State

Additional Authors: Marius Roos, Marlene Schoeman, Louis Holtzhausen, Gina Joubert

\section{Exercise Prescription by South African Doctors Part 1: Knowledge, Practice and Attitudes Among South African Doctors4}

Introduction: Physical inactivity is the fourth leading cause of death worldwide. South Africans have low physical activity (PA) levels which increase the morbidity and mortality associated with various chronic diseases. General Practitioners (GPs) play a key role in motivating a large proportion of the sedentary population to become physically active. Very little is known about the exercise prescription practices of South African GPs. This study aimed to determine the practices, attitudes toward and knowledge on exercise prescription among GPs in SA and identify possible barriers why they do not prescribe exercise.

Methods: A self-administered, anonymous electronic questionnaire was circulated to a database of GPs via email on three separate occasions, two weeks apart and was completed by a total of $349 \mathrm{GPs}$. Exercise prescription practices, attitudes towards exercise prescription and the importance thereof as preventative modality for chronic diseases were assessed. Knowledge on benefits, risk factors, contraindications and compilations of exercise prescriptions were also assessed.

Results: Substantially higher prescription rates were reported compared to international literature. A minority (18.0\%) of the participants felt that exercise prescription will be too time consuming, while almost half $(46.0 \%)$ of the non-prescribing doctors reported a lack of confidence in their knowledge to be able to prescribe exercise. Approximately $98 \%$ of the GPs believed that it should be part of their practice to prescribe exercise to their patients, despite the fact that their knowledge regarding recommendations for physical activity and the formulation of an exercise prescription was poor.

Conclusion: Although GPs reported a high prevalence of exercise prescription, insufficient knowledge about exercise prescription and appropriate lifestyle modifications were noticed. Barriers to exercise prescription differ from international literature and should be investigated further. A lack in confidence and knowledge to enable safe and effective exercise prescription highlights a need to rethink the undergraduate medical curricula

Takshita Sookan

Affiliation: University of KwaZulu Natal

Additional Authors: Takshita Sookan, Andrew McKune, Michael Ormsbee, Jose Antonio, Nombulelo Magula, Umesh Lalloo, Ayesha Motala

\section{Effect of a progressive resistance training program and whey protein intake on quality of life in human immunodeficiency virus infected} individual receiving antiretroviral therapy.

Introduction: Advances in HIV treatment in the last three decades has resulted in improved health, prolonged life and substantially reduced the risk of HIV transmission. [u1] This impact broadens to all facets of life, influencing quality of life (QOL). Progressive resistance training (PRT) combined with effective supplementation can increase muscle mass and improve physical performance in persons with HIV infection and may enhance outcomes in these patients as well as improve QOL.

Methods: Forty HIV infected participants $(40.8 \pm 7.7 \mathrm{yrs}, 70.8 \pm 16 \mathrm{kgs}$, BMI $30.9 \pm 7.2 \mathrm{~kg} . \mathrm{m} 2)$ receiving ART ( $\geq 18$ months) were randomly assigned to either a whey protein/resistance training (RT) group $(n=18)$, placebo/PRT group $(n=14)$ or control group $(n=8)$. Participants received either 20g whey or placebo (maltodextrin) pre and immediately post each RT workout. Whole body RT was performed 2/week for 12 weeks. To assess QOL the WHOQOL-HIV BREF 31 was used which has six domains: physical, psychological, level of independence, social relationships, [u2] environment, and spiritual. The questionnaire was completed at baseline and then at 12 weeks. The mean score of questions within each domain was used to calculate the domain score. Statistical analysis consisted of a two-way ANOVA and Sidak's multiple comparison post hoc testing. Alpha was set at $P$ $\leq 0.05$.

Results: The Physical Domain showed a significant time effect (TE) $(P=0.02)$ with the placebo group increasing from baseline (17.69a.u.) to post (19.15a.u) (Mean difference -1.5a.u., 95\% Cl -2.9 to -0.05a.u.). There were no significant changes in the supplement group. The Social Relationships Domain exhibited a significant TE $(\mathrm{P}=0.02)$ with the placebo group increasing significantly from 14.23a.u. to 16.54a.u post (Mean difference -2.3a.u., $95 \% \mathrm{Cl}-3.9$ to -0.7 a.u. $)$. Environment Domain showed a significant TE $(\mathrm{P}=0.002)$ with both placebo (15.65a.u. to 16.46a.u.; mean difference -0.8 a.u., $95 \% \mathrm{Cl}-1.5$ to -0.09 a.u.) and supplement groups (14.35a.u. to $15.03 a . u$.; mean difference -0.7 a.u., $95 \% \mathrm{Cl}-1.3$ to 0.04a.u.) demonstrating significant increases from baseline after 12 weeks of training. The Spiritual Domain indicated significant TE ( $P=0.05)$ with the placebo group increasing from baseline (16.38a.u.) to post (17.92a.u) (Mean difference $-1.5 a . u ., 95 \% \mathrm{Cl}-2.8$ to $-0.3 a . u$.). There were no significant changes in the supplement group. There were no changes in the Psychological or Level of Independence Domains for the placebo and supplement groups. There were no significant changes in the control group for any of the six domains.

Conclusion: Several components of QOL improved in ART treated HIV infected individuals that participated in the PRT program. Changes were predominately shown in the placebo group (Domains $1,4,5,6$ ). This can be attributed to positive social and environmental effects of exercise programs. Exercise training is an inexpensive and efficacious strategy for improving QOL in this population with can impact other facets of their lives.

Jason Tee

Affiliation: University of Johannesburg

Additional Authors: Jason Tee, Jannie Klingbiel, Rob Collins, Mike Lambert, Yoga Coopoo

\section{Functional Movement Screen predicts severe contact and non-contact injuries in professional rugby union players}

Introduction: Rugby union is a collision sport with a relatively high risk of injury. The ability of the Functional Movement ScreenTM (FMS) to predict the occurrence of severe ( $\geq 28$ days) contact and non-contact injuries in professional players was assessed.

Methods: 90 FMS test observations were compared with severe injuries sustained during 6 subsequent months. A receiver operated characteristic (ROC) curve determined the FMS score that best predicted severe injury. $2 \times 2$ contingency tables were used to determine sensitivity, specificity and odds ratios of the prediction.

Results: Mean FMS scores were significantly lower in players who sustained severe injury (injured $13.1 \pm 1.7$ vs. non-injured $14.5 \pm 1.4$ ), and severe contact injuries (injured $13.1 \pm 2.0$ vs. non-injured $14.3 \pm 1.5$ ). A receiver-operated characteristic (ROC) curve determined that odds of severe injury, contact injury and non-contact injury were $5.2(95 \% \mathrm{Cl}=2.0-13.9), 6.5(95 \% \mathrm{Cl}=1.8$ to 23.0$)$ and $4.3(95 \% \mathrm{Cl}=0.9$ to 21.0$)$ times greater respectively if FMS score was below the relevant cut-off score. Low active straight leg raise score $(\leq 2)$ was also significantly associated with injury. Players were divided into High-FMS $(\geq 14)$ and Low-FMS $(\leq 13)$ groups to estimate the potential effect of low FMS scores. Survival analysis showed a greater fractional survival rate for High-FMS group $(\geq 14)$ versus a Low-FMS group $(\leq 13)(81.4 \% 95 \% \mathrm{Cl}=68.9$ to $89.2 \% \mathrm{vs} .50 .0 \% 95 \% \mathrm{Cl}=31.3$ to $68.7, \mathrm{p}$ $<0.05)$. The Low-FMS group spent mor e days injured ( $47 \pm 47$ vs. $25 \pm 39$ days) and took longer to recover ( $29 \pm 38$ vs. $10 \pm 16$ days) than the HighFMS group.

Conclusion: These findings indicate that FMS score is a risk factor for severe contact and non-contact injury in professional rugby players. 


\section{Movement, impact and pacing characteristics of South African professional rugby union players}

Introduction: Global positioning system (GPS) technology provides accurate, real-time movement pattern analysis. GPS will improve understanding of the movement characteristics of players during professional rugby union matchplay.

Methods: 19 Professional rugby players were tracked using GPS during 24 matches during the 2013 rugby season. Players were grouped as (1) backs or forwards and (2) tight forwards, loose forwards, scrumhalves, inside backs or outside backs. Movements were categorized as walking (0$2 \mathrm{~m} . \mathrm{s}-1)$, jogging $(2-4 \mathrm{~m} . \mathrm{s}-1)$, striding $(4-6 \mathrm{~m} . \mathrm{s}-1)$ and sprinting $(>6 \mathrm{~m} . \mathrm{s}-1)$. Walking and jogging were classified as low intensity and striding and sprinting as high intensity movement zones. An inbuilt tri-axial accelerometer quantified impacts. Pacing effects were determined by comparing 1 st and 2nd half movement patterns and whole game players to substitutes.

Results: There was no difference between forwards and backs in relative distance covered or impact variables. Backs reached higher maximum speeds than forwards (Backs $8.8 \pm 1.1 \mathrm{vs}$. Forwards $7.6 \pm 1.3 \mathrm{~m} . \mathrm{s}-1$, ES $=1.0$ ), and covered more distance than forwards in high intensity speed zones (forwards $10 \pm 5 \mathrm{vs}$. backs $12 \pm 4 \mathrm{~m}$. min-1, ES $=0.6$ ). Outside backs were the fastest positional group $(9.4 \pm 0.9 \mathrm{~m} . \mathrm{s}-1, \mathrm{ES}=0.4-2.2)$, while tight forwards covered the most distance in low intensity zones (57 $\pm 5 \mathrm{~m} \cdot \mathrm{min}-1$, ES $=0.4-1.8)$ Loose forwards and inside backs exhibited similar movement patterns. Relative distance (m.min-1) increased in the second half for whole game players (7 \&p lusmn; $9 \%$ ). High-intensity running distance decreased in the second half for forwards ( $1 \mathrm{st} 10.0 \pm 5.0 \mathrm{vs}$. 2nd $8.6 \pm 3.8 \mathrm{~m}$. min-1), but increased for backs ( $1 \mathrm{st} 10.6 \pm 3.2 \mathrm{vs}$. 2nd $11.4 \pm 2.5$ $\mathrm{m} . \mathrm{min}-1$ ). Substitutes demonstrated increased relative jogging (Start $20 \pm 5 \mathrm{vs}$. Sub $24 \pm 6 \mathrm{~m}$.min-1) and striding (Start $8 \pm 2 \mathrm{vs}$. Sub $11 \pm 6 \mathrm{~m}$. min-1) distance versus whole game players.

Conclusions: There are notable differences in the movement of professional rugby union players in different positions. Fatigue results in reduced high-intensity running distance and this effect can be managed through the use of substitutes.

Hannah van Buuren

Affiliation: ISR, University of Pretoria

\section{Methods in monitoring a fast bowler in Cricket}

Introduction: Fast Bowlers cover $20-80 \%$ greater distance, exert 2-7 times greater high intensity (HI) distances, and have $35 \%$ less recovery time between $\mathrm{HI}$ efforts than other specialities in cricket. In addition, they are also required to undergo and absorb forces as high as $8 \times \mathrm{BW}$ during each bowling delivery whilst laterally flexing, extending and rotating throughout their bowling action. It is no surprise that fast bowlers have been identified as the speciality highest at risk of injury in various countries. The aim of this data collection was to determine methods that can be used in practice by support staff to determine when the fast bowler may be at risk of injury and whether or not the fast bowler is bowling prepared prior to competition.

Methods: Thirteen international fast bowlers were monitored for an entire cricket season by self-reporting their workloads using number of overs bowled, session types and time, as well as injury occurrence and time to play. Results: The average number of balls bowled per week over the season was $120 \pm 39$ balls with acute peaks reaching 258 balls in one week. Conditioning on average for the bowling group was $169 \pm 14$ minutes per week. Trends from injuries sustained showed that muscle strain type injuries occurred over a short delay ( 1 week) between when the bowler experienced high workloads and breakdown where they could not play cricket due to injury. Bone and tendon damage were seen over a long delay (36 week) between high workloads and breakdown. High workloads were experienced when the fast bowler spiked acutely to their chronic (rolling average of 3 weeks) workload.

Conclusion: This method of monitoring training loads will allow coaches and trainers to either lower or raise bowling and /or conditioning loads prior to competition or risk of injury, e.g. to ensure bowling loads have met the demands for the upcoming test match, or to ensure the fast bowler is fresh and at a reduced risk of injury.

Riaan Van der Merwe

Affiliation: Morningside Sports Medicine Centre

Additional Authors: Riaan Van der Merwe, Natalie Phillips

\section{Isokinetics, Posture and Functional Screening in Professional Cyclists-Team MTN Qhubeka}

Introduction: An elite level cyclist rides between $25000 \mathrm{~km}$ and $35000 \mathrm{~km}$ per annum; compared to a recreational cyclist who only accumulates 7114 $\mathrm{km}$. It is therefore surprising that so few studies exist to illustrate the findings of isokinetic, functional and postural testing modalities in elite cyclists. The purpose of this study is to present data collected in an elite group of cyclists.

Methods: Twenty one professional road cyclists from the MTN Qhubeka cycling team were screened prior to competing in the 2015 Tour de France. The screening protocol included isokinetic testing, postural analysis, functional strength testing and flexibility of the lower body. Isokinetic examination was performed using a System 3 Biodex. Peak torque to body weight ratios were evaluated at 60 degrees/s and 180 degrees/s for concentric knee extension/flexion and 60 degrees/s and 120 degrees/s concentric hip flexion/extension. Postural analysis involved documenting asymmetry of bony landmarks to determine malalignment issues. Functional evaluation included single leg squat and single leg jump squat tests. Goniometry was used to evaluate hip internal, external rotation and passive hamstring flexibility.

Results: The cyclists' average age was 27.3 years with a mean body weight of $71.6 \mathrm{~kg}$. Isokinetic testing revealed the hip extensors and knee flexors to be the stronger muscle groups. Results indicated average peak TQ/BW for knee flexors to be very strong $(\mathrm{L}=3.08 \mathrm{~nm} / \mathrm{kg}, \mathrm{R}=3.0 \mathrm{~nm} / \mathrm{kg}) \mathrm{compared}$ to general athletes' ratio of $1.85 \mathrm{~nm} / \mathrm{kg}$. However, of concern was the weakness in knee extensor strength - average peak $T Q / B W$ ratio $(L=2.22$ $\mathrm{nm} / \mathrm{kg}, \mathrm{R}=2.27 \mathrm{~nm} / \mathrm{kg}$ ) compared to general athletes of $2.96 \mathrm{~nm} / \mathrm{kg}$. Postural assessment indicated that only 2 cyclists had a neutral pelvic position and 3 cyclists' scapulae were level. During functional testing $4 \%$ had good stability on L and R leg (ankle, knee and hip) during a single leg squat, $14 \%$ had good stability on 1 leg only (Left) with the 1 leg jump squat only $14 \%$ presented good stability on both legs. Flexibility tests illustrated that hip internal rotation (average: $\mathrm{L}=35.57 \mathrm{a}^{\circ}, \mathrm{R}=35.05 \mathrm{â}^{\circ}$ ) and hamstring flexibility (average: $\mathrm{L}=87.76 \mathrm{a}^{\circ}, \mathrm{R}=88.95 \mathrm{a}{ }^{\circ}$ ) proved in line with norms, yet the average hip external rotation $\left(L=30.67 \hat{a} \quad{ }^{\circ}, R=30.62 \hat{a} \quad{ }^{\circ}\right)$ was significantly reduced compared to the norm $\left(45 \hat{a}{ }^{\circ}\right)$.

Conclusion: These athletes could be at risk of injury considering the results. The performance of the team could be improved by enhancing knee extensor strength and addressing postural mechanics. Cycling is a non-weight bearing sport thus the poor performances in functional testing. 


\section{Interventions preventing ankle sprains; predictors of compliance to neuromuscular training, bracing or combination therapy}

Introduction: Neuromuscular training, wearing a brace or a combination of both are the most occurring interventions for preventing reoccurring ankle sprains in sports. The effectiveness depends on compliance to the intervention. The aim of this study was to describe the associations between participants' person-related potential predictor variables and cumulative compliance with interventions for preventing ankle sprains: neuromuscular training, wearing an ankle brace, and a combined training and bracing.

Design: Secondary analysis of compliance data from a randomized controlled trial (RCT) comparing measures preventing ankle ligament injuries. Methods: Ordinal regression with a backward selection method was used to obtain a descriptive statistical model linking participants' person-related potential predictor variables with the monthly cumulative compliance measurements for three interventions preventing ankle ligament injuries.

Results: Having had multiple previous ankle injury versus a single ankle injury was significantly associated with a higher compliance with all of the preventive measures (OR 1.72; 95\% Cl 1.09-2.70). Overall compliance with bracing and the combined intervention was significantly lower than the compliance with NM training. Per group analysis found that participating in a high-risk sport, like soccer, basketball, and volleyball, was significantly associated with a higher compliance with bracing (OR 3.39 95\% Cl 1.49-7.44), or a combined bracing and NM training (OR 2.49 95\% Cl 1.27-4.92). In contrast, participating in a high-risk sport was significantly associated with a lower per group compliance with NM training (OR $0.4395 \% \mathrm{Cl} 0.19-$ 0.96).

Conclusions: When choosing an appropriate intervention to prevent reoccurring ankle sprains practitioners should take into account the nature of the sport a patient practices. Furthermore, it is advisable to pay extra attention to patients who are returning to sports after their first ankle sprain to stimulate compliance to the chosen intervention.

\section{Arnold Vlok}

Affiliation: Department Exercise and Sports Sciences

Additional Authors: Arnold Vlok, Louis Holtzhausen, Marlene Schoeman

\section{Rehabilitation and return to play after anterior cruciate ligament reconstruction: a Systematic review and development of a conceptual} rehabilitation framework

Introduction and aim:There is a high risk for subsequent injury after Anterior Cruciate Ligament Reconstruction (ACLR) when post-operative rehabilitation is not managed according to physiological principles. The primary aims of this study was 1) to conduct a systematic literature review on the influence of the physiological healing process of the graft on the physical rehabilitation process leading up to return to sport (RTS) following ACLR, and 2) to develop a conceptual framework for rehabilitation and RTS (Ethics clearance: ECUFS 15/2013).

Methodology: A systematic review was conducted according to the PRISMA guidelines. Randomised controlled trials (RCTs), prospective cohort (PC), cross sectional (CS) studies and descriptive epidemiological studies from 1985 to 2013 were reviewed. Electronic databases which were searched included MEDLINE, Academic Search Complete, Pubmed and SPORTDiscus. Twenty eight articles were selected for data extraction, which were categorised in three main categories, namely graft healing (5 articles), rehabilitation protocols ( 15 articles) and return to sport criteria ( 8 articles).

Results: There is a dearth of literature aligning the physiological healing process after ACLR and the physical rehabilitation thereof. Major differences occur in methodology and in rehabilitation protocols in the literature. Physiological healing of the autograft and quadriceps recruitment deficits take up to 2 years for full restoration. Rehabilitation interventions after ACLR include strength, neuromuscular and functional training according to the physiological responses of the ACL graft and neuromuscular adaptation. Current trends in rehabilitation planning and intended outcomes follow these principles. Weakness or asymmetry of the injured versus uninjured side often persists after RTS.

Conclusion: A 0-3 months/3-6 months/6-12months/RTS conceptual framework for rehabilitation of ACLR was developed from the literature, considering the interdependence of the healing process of the ACL graft and neuromuscular, strength and functional training. ACLR patients need to be managed within a time based as well as individualized criterion based rehabilitation progression model.

\section{Estelle Watson}

Affiliation: Centre for Exercise Science and Sports Medicine, School of Therapeutic Sciences, Faculty of Health Sciences, University of the Witwatersrand

Additional Authors: Estelle Watson, Lisa Mickelsfield,

\section{Are our pregnant mom's exercising for two? Physical activity profiles of black South African pregnant women}

Background: Excessive gestational weight gain (GWG) has been shown to be an predictor of adverse maternal outcomes, such as gestational diabetes mellitus, preeclampsia and caesarean delivery. It is however, one of the most important modifiable risk factors for pregnancy complications. Unsurprisingly, engaging in physical activity during pregnancy has been shown to reduce excessive GWG, and therefore may provide a protective health effect. In South Africa, women from low socio-economic urban areas are particularly susceptible to issues of obesity and inactivity. Therefore, understanding the activity and health profiles of this vulnerable population is a crucial step to improving the health of our women and future generations.

Methodology: Pregnant women (14-18 weeks gestation) attending the Developmental Pathways of Health Research Unit at Chris Hani Baragwaneth Hospital in Soweto were invited to participate in the study. Demographic information was gathered through questionnaire, and anthropometric measurements were taken by trained research assistants. Physical activity was measured using a hip-worn accelerometer (ActiGraph GXT3) for 7 days.

Results: One hundred and ten women participated in the study ( $\mathrm{N}=110$; age: $30 \pm 6 \mathrm{yrs})$. On average, women weighed $69.8( \pm 13.8 \mathrm{~kg})$, and had a body mass index of $27.1( \pm 4.9)$ at $14-18$ weeks. Gestational weight gain over the course of pregnancy was $8.4( \pm 4.7 \mathrm{~kg})$. Objective measured showed that sixty six women $(60 \%)$ were sufficiently active, meeting the 150 minutes of MVPA per week, however, women spent on average $7.3( \pm 2.8 \mathrm{hrs} / \mathrm{day})$ in sedentary pursuits.

Discussion: Many black South African pregnant women are not sufficiently active, and spend the majority of their day in sedentary activities. In addition, this research suggests that many women falling pregnant are already overweight, and therefore early lifestyle interventions may be critical in preventing disease in this population. The health risks of overweight and a sedentary lifestyle may extend to birth outcomes for the baby and postpartum outcomes for the mother. Therefore, public health interventions to improve activity levels, in this unique period in a women's life, is warranted. 
Ming Hao Zheng

Affiliation: Winthrop Professor and Director of Research at the Translational Orthopedic Research Centre, Sir Charles Gardner Hospital, Perth and the Associate Dean (International) of the Faculty of Medicine, Dentistry and Health Sciences at the University of Western AustraliaAdditional Authors:

Additional authors: Allan Wang, Ming Hao Zheng

\section{Evidence of Long Term Durability on Autologous Tenocyte Injection (Ati) for Treatment Of Chronic Lateral Epicondylitis}

Purpose: Chronic lateral epicondylitis is associated with degenerative tendon changes, extracellular matrix breakdown and tendon cell loss. To continue our previous 12 month pilot study on ATI for severe tendinopathy associated with chronic lateral epicondylitis (Wang et al AJSM 2013), herein we provide long-term follow up (36 months) data on ATI efficacy.

Methods and Materials: Patients with severe refractory lateral epicondylitis underwent clinical evaluation and MRI prior to intervention. A patellar tendon needle biopsy was performed and tendon cells expanded by in vitro culture in a GMP-certified TGA-licensed facility. As single injection of autologous tenocytes into the common extensor tendon origin tendinopathy under ultrasound guidance was performed. Patients underwent serial clinical evaluations (VAS pain, QuickDASH, grip strength and MRI) for up to 5 years post-injection. Repeat MRI scanning was performed at 1 year and at least 3 years post-injection.

Results: Twenty patients (11M:9F; mean age 49.4 years) were included in the study, with three patients withdrawing consent prior to ATI. Mean symptoms duration pre-recruitment was 31 months. Mean follow-up time was 4.5 years. No biopsy complications or any adverse events, infection or excessive fibroblastic reactions at the injection site were observed. One patient elected for surgery three months post-ATI after re-injury, and one died of natural causes during follow-up. In the remaining 15 patients, mean pain scores improved from 5.7 to 1.2 at final follow-up ( $p<0.001$ ). Mean QuickDASH score and grip strength scores also significantly improved over follow-up ( $84 \%$ and $207 \%$ increase, respectively; $p<0.001)$. MRI scoring of tendinopathy grade at the common extensor origin improved significantly at 12 months $(p=<0.001)$, and was maintained to final follow-up. Conclusion: ATI significantly improved clinical function and MRI tendinopathy scores at 3-year follow up in patients with chronic lateral epicondylitis having previously undergone unsuccessful conservative treatment. This study advocates the long term durability of ATI in treating tendinopathy.

Ming Hao Zheng

Affiliation: Affiliation Winthrop Professor and Director of Research at the Translational Orthopedic Research Centre, Sir Charles Gardner Hospital, Perth and the Associate Dean (International) of the Faculty of Medicine, Dentistry and Health Sciences at the University of Western Australia Additional Authors: Greg Janes, Ming Hao Zheng

\section{Autologous Tenocyte Injection (ATI) for Gluteal Tendinopathy: a Pilot Study}

Introduction: Gluteal tendinopathy is a common cause of lateral hip pain. No treatments have effectively improved the poor health outcomes and disability of the condition. Autologous tenocyte injection (ATI) is a novel cell therapy that has shown promise in other tendinopathic conditions. Therefore, this prospective pilot study investigated the effect of ATI in 12 patients with clinical and radiological evidence of gluteal tendinopathy.

Method: A patellar tendon needle biopsy was performed under local anaesthetic and tendon cells were expanded by in vitro culture in a GMPcertified, TGA-licensed facility. Autologous tenocytes were injected into the gluteal tendinopathy under ultrasound guidance on a single occasion. All patients were functionally assessed preoperatively and at 3, 6, 12 and 24 months postoperatively with the Oxford Hip Score (OHS), the Merle d'Aubigne Postel Score (MDP), the 36-item Short-Form Health Survey (SF36) and the Visual Analogue Pain Scale (VAS). A patient satisfaction survey was also given 12 months postoperatively. Magnetic Resonance Imaging (MRI) scans were performed preoperatively and 6 months postoperatively for structural assessment of the gluteus medius tendon.

Results: Twelve patients, all female, with a mean age of 53 (range 40-65) years and average duration of symptoms of 33 months (range 6-144) were included in the study All patients had clinical symptoms and signs of gluteal tendinopathy, with diagnosis confirmed by MRI all patients. No patella biopsy site complications or treatment site infections were noted. Significant $(p<0.05)$ functional improvement to 24 months postoperative was observed across all mean score outcomes: VAS (pre 7.25, post 2.73), OHS (pre 24.00, post 39.45), MDP (pre 11.67, post 16.55) and SF-36 PCS (pre 28.08, post 41.59). One patient did not respond and elected surgery. Patient satisfaction survey results demonstrated that $64 \%$ of patients were 'satisfied' or 'highly satisfied' with their ATI outcome. Follow-up MRI scans did not demonstrate notable changes in the radiological appearance of th e tendinopathic tissue in most cases.

Discussion: ATI by single injection significantly improved clinical outcome in this pilot study of gluteal tendinopathy at 24 months follow-up. However, the safety and efficacy of ATI requires substantiation given the small pilot sample size. We believe this study has shown encouraging early outcomes that warrant larger randomised controlled study of ATI for gluteal tendinopathy. 


\section{Abstracts: E-Posters}

Marelise Badenhorst

Affiliation: Division of Exercise Science and Sports Medicine, Department of Human Biology, Faculty of Health Sciences, UCT

Additional Authors: Marelise Badenhorst Prof Willem van Mechelen, Prof Mike Lambert, Prof Evert Verhagen, Wayne Viljoen, Clint Readhead, Gail Baerecke, Chris Burger, Petro Jackson, James Brown

\section{A comparison of rugby-related catastrophic spinal cord injury rates by Province in South African Rugby}

Introduction: The catastrophic injury rates in South African rugby have previously been reported at an "acceptable" level of risk (0.1-2.0 cases per 100000 players). To further reduce this risk, the South African Rugby Union (SARU) requires that all coaches and referees undergo biennial training as a core component of SARU's dedicated rugby safety programme, BokSmart. There are approximately 300000 players from 14 different rugby regions (< rugby regions ('Provinces") in South-Africa. Understanding the difference in catastrophic injury risk between the provinces is important for a nationwide intervention such as BokSmart. Thus, the aim of this study was to compare the catastrophic injury rate between the 14 South African rugby Provinces between 2008 and 2014.

Methods: Acute spinal cord injuries (ASCI) with permanent outcomes (neurological deficit, quadriplegia or fatal) were obtained from BokSmart/Chris Burger Petro Jackson Players' Fund (CBPJPF). The player numbers in each Province were obtained from SARU's 2013 Census report. Annual average incidence rates between 2008 and 2014 were calculated for each Province and compared statistically using a Poisson regression with a $95 \%$ level of confidence $(P<0.05)$.

Results: The overall incidence rate for permanent outcome ASCI was 2.33 (95\% Cl: 0.34 to 4.32$)$ per 100000 players.

David Bentel

Affiliation: Centre for Exercise Science and Sports Medicine, School of Therapeutic Sciences, Faculty of Health Sciences, University of the Witwatersrand

Additional Authors: David Bentel, Khavish Harry, Marc Booysen

\section{The anthropometric and physical performance characteristics of second division male footballers from Gauteng}

Background: There is a paucity of research concerning the anthropometric and physical performance characteristics of male footballers from South Africa according to player position.

Aim: The aim was to describe the anthropometric and physical performance characteristics of male footballers playing in the second division (Gauteng stream) according to player position.

Methods: One hundred and twenty four male footballers (Age: $22.1 \pm 2.72$ years) $($ Strikers $=30$; midfielders $=40$; defenders $=39$; goalkeepers $=15$ ) from five football teams performed the following anthropometric and physical performance measurements: body mass, stature, upper body power (overhead medicine ball throw), lower body power (countermovement jump), agility (Illinois test with ball), speed (10 and $30 \mathrm{~m}$ sprints) and intermittent running endurance (Yo-Yo Level 1).

Results: Goalkeepers were heavier than strikers and midfielders $(P=0.002)$ and strikers were shorter in stature than goalkeepers and defenders $(P$ $=0.003)$. Goalkeepers and defenders had greater upper body power than forwards and midfielders $(P=0.0002)$, however goalkeepers ran less distance in the Yo-Yo IR1 than midfielders and strikers $(P=0.001)$. No positional differences were observed for countermovement jump height, agility and sprints $(10 \mathrm{~m}$ and $30 \mathrm{~m})$ (P values $=0.14 ; 0.06 ; 0.24 ; 0.18$, respectively).

Conclusion: The results suggest that there is little heterogeneity regarding the physical performance characteristics of male second division footballers (Gauteng) in the outfield position. It is recommended that individualising physical conditioning based on the positional demands may need to be addressed in this league.

Keywords: Soccer, football, anthropometric, counter movement jump, Yo-Yo, sprints

Gerrit Jan Breukelman

Affiliation: University of Zululand

Variable isotonic resistance training in lower limb strength and flexibility of recreational cyclists and runners

We determined the effects of an 8-week, home-based Jump Stretch Flexband® (JSFB) exercise programme on the strength and flexibility of a group of recreational runners and cyclists, with a control group of runners and cyclists who did not alter their daily training regimes. The use of the JSFB significantly increased the dominant and non-dominant hip flexor flexibility of the experimental group ( $p=0.0002$ and 0.0004 , respectively). Significant benefits were also found in hamstring flexibility $(p=0.041)$, evidence indicating a steady increase within the experimental group. There was also a significant, but inconclusive, increase in the control group's quadricep flexibility in both dominant and non-dominant legs $(p=0.025$ and 0.046). There was no significant difference in strength gains, as the experimental group's peak torque-best repetition for extension of the dominant leg was $p=0.37$ and the non-dominant leg was $p=0.19$, respectively. Thus, using the JSFB appears likely to lead to improved flexibility of an athlete's lower limb muscles, which is an important finding due to the significantly less amount of research that has been done on the large muscle groups compared to the smaller muscle groups. 


\section{Do shoulder stability exercises create similar increases in the acromiohumeral distance of cricket players as the scapular assisted test?}

Introduction: Prolonged participation in overhead sports creates shoulder muscle imbalances that eventually alter the efficacy of the shoulder stabilizer muscles and heighten injury risk such as subacromial impingement syndrome. The aim of this study was to determine if a six week shoulder stability exercise intervention performed during the competitive season results in similar increases in the acromiohumeral distance (AHD) of cricket players as with the application of the scapular assisted test (SAT).

Method: Players $(\mathrm{N}=47)$ from the North-West University cricket squad voluntarily participated in this shoulder injury prevention project. Baseline testing included AHD measurements performed by a radiographer at different humeral abduction angles $\left(0^{\circ}, 30^{\circ}\right.$ and $\left.60^{\circ}\right)$ with and without the application of the SAT. Players were then randomly assigned to an intervention and control group. The control group continued with their normal inseason programme. The intervention group additionally performed shoulder stability exercises twice a week for six weeks under supervision of a Biokineticist.

Results: The SAT application had a significantly greater effect at $60^{\circ}$ of humeral abduction on the dominant shoulder $(p=0.013)$ compared to the nondominant shoulder. Also, the dominant shoulder indicated significantly less narrowing of the AHD between $0^{\circ}$ and $60^{\circ}$ ( $p \leq 0.001$ ), as well as $30^{\circ}$ and $60^{\circ}(p \leq 0.001)$ ranges when the SAT was applied. The players in the intervention group indicated similar widening of the AHD at $0^{\circ}$ and $30^{\circ}$ humeral abduction angels as the AHD measures with the SAT application. The exercise intervention on average significantly widened the AHD of both the dominant $(p=0.019)$ and non-dominant shoulders $(p=0.035)$ at $0^{\circ}$ abduction.

Conclusion: Shoulder stability exercises performed additional to normal training regimes during the competitive season result in similar widening in the AHD of cricket players at $0^{\circ}$ and $30^{\circ}$ of humeral abduction than when the SAT is applied. These exercise modalities could therefore be incorporated in the training regimes of overhead athletes to manage shoulder dyskinesis.

Keywords: Shoulder dyskynesis and overhead athletes, acromiohumeral distance, shoulder dyskinesis rehabilitation

\section{Tanya Camacho}

Affiliation: University of Pretoria

\section{The Correlation between Chest Circumference and Spirometry Measurements over a 19-week Intense Training Programme}

Introduction: Spirometry testing as a screening tool for pulmonary disease is expensive and often inaccessible. This study aimed to determine whether chest circumference measurements could be used as an inexpensive pre-screening tool for pulmonary disease irrespective of changes in fitness.

Methods: A quantitative, prospective correlational research design assessing chest circumference measurements, spirometry and maximal oxygen uptake (VO2 max) of male and female South African recruits $(n=235)$ undergoing 19 weeks of Basic Military Training (BMT) was used. Subjects were tested in weeks 1,12 and 19 of BMT. Overall, 26 subjects matched the inclusion criteria and completed all measures in all three testing sessions. Parametric descriptive and inferential statistics were used.

Results: Data analysis showed weak correlations $(r<0.4)$ between chest circumference and spirometry. Significantly positive strong correlations between forced vital capacity (FVC) and VO2 max in weeks 1,12 and $19(r=0.682, p<0.01 ; r=0.616, p<0.01$ and $r=0.697 ; p<0.01$, respectively) and between forced expiratory volume in 1 second (FEV1) and VO2 max in weeks 1 and 19 of BMT $(r=0.628 ; p<0.01$ and $r=0.658 ; p<0.01$, respectively) were observed. Additionally, a significantly positive moderate correlation between FEV1 and VO2 max in week 12 of BMT ( $r=0.554 ; p<0.01$ ) was noted.

Conclusions: This study demonstrates that chest circumference measurements appear not to be a reliable substitute for spirometry screening in a young healthy active population. Future research using a larger sample is recommended.

Keywords: pulmonary disease; chest circumference measurements; spirometry; forced vital capacity; forced expiratory volume in one second; basic military training

James Clark

Affiliation: Department Physiology, University of Pretoria

Additional Authors: James Clark, Maegan Anne Von Finckenstein, Mareli Joubert, Danielle Lincoln

\section{A Retrospective Study of Injuries in Rowing During a Single Season}

Introduction: Sport injury profiling from epidemiological data provides important information for preventive and management strategies by scientific and medical support teams. Competitive rowing demands consistent, long-term intensive training, and physical injury represents both a risk of and a threat to optimal preparation. The aim of this study was to retrospectively document injuries sustained by competitive rowers in South Africa over one full season in an effort to obtain a baseline injury profile of the sport.

Methods: At a season-ending national regatta, one hundred and fifty-seven (157) rowers (88 men, 69 women) aged (mean \pm SD) $21.2 \pm 2.1$ years, with $5.0 \pm 3.5$ years rowing experience, representing nine university rowing clubs from across South Africa volunteered as participants. A three-part questionnaire containing closed-ended questions on personal data, rowing training and injury information pertaining to that season was completed. Participant competitive level ranged from university club representatives to international medal winners.

Results: Eighty (80) rowers (50.9\% of respondents) admitted sustaining at least one injury requiring one or more missed training sessions, with 103 separate injuries reported. Lower back $(23.3 \%)$, knee $(13.6 \%)$ and shoulder $(10.7 \%)$ were the most commonly injured regions within a variety of involved sites. The vast majority $(68.9 \%)$ of injuries were sustained during training as opposed to competition, and more often during rowing (42.7\%) than other training modalities. Approximately $30 \%$ of cases represented a recurrence of a prior injury. Muscle strain (48.5\%) was the most reported type of injury. An injury typically resulted in 7.0 (range: $0.5-180.0$ ) days of missed training. While national representative rowers made up less than one fifth of participants they reported over one third of the injuries documented.

Conclusions: A high percentage of rowing injuries in South Africa involve the lower back, congruent with international data. Furthermore, overuse injury owing to training load is implicated most often in the aetiology based on the reported nature and modality of injury and the higher injury incidence amongst rowers at higher competitive levels. This data has important practical utility in strategies aimed at managing athlete health and optimizing preparation time in the scientific support program to the national rowing squad. 


\section{Prevalence of overweight in Grade-One Learners And Parent Perceptions of Childhood Nutrition / Physical Activity}

The problem of childhood obesity in South Africa has reached epidemic proportions. It is estimated that one in five South African children are either overweight or obese; with twenty percent of children under the age of six being overweight. This is mainly due to a poor diet and a lack of exercise. The aim of this study is to determine the overweight/obesity prevalence amongst grade-one learners at selected schools in the West Rand, Mogale City. The weight and height of each subject was to be physically measured by the researcher and compared to norms for that age category. This study further aims to determine their parents knowledge/perceptions regarding childhood nutrition and physical activity. To this end a questionnaire was constructed so that parental knowledge/beliefs could be assessed. This study has found both overweight and underweight within the same population. The results indicate overweight/obesity in seventeen subjects $(3.7 \%)$. Eleven girls $(4.8 \%)$ and six boys $(3 \%)$ were overweight representing a boy to girl ratio of $1: 1.8$ among the overweight group. Among the overweight subjects, girls represented $65 \%$ while boys represented $35 \%$. This study has also found underweight/stunting of growth among the eight and nine year old subjects as their weight for height fell below the 25th percentile. Further classification of the study sample according to school-fee structure revealed that all subjects with overweight/obesity were found within low-fee schools, representing $4 \%$. One boy and one girl each were found with obesity among the overweight group having a body mass index (BMI) of 23.8 and 24.8 respectively. Therefore obesity was found in $12 \%$ among the overweight group and within lowfee structure schools.

\section{Shereen Currie}

Affiliation: University of Zululand

\section{Comparison of lifestyle habits, sleep and activity patterns between normal, overweight and obese individuals using the BodyMediaßFITArmband.}

The aim of this study was to compare the lifestyle habits, quality of life, sleep and activity patterns between normal, overweight and obese individuals using the BodyMedia®FITArmband. Thirty (30) participants volunteered to participate in this study, and were divided into three groups. The first group consisted of normal weight $(n=8)$ participants (BMI $18.5-24.9 \mathrm{~kg} / \mathrm{m} 2)$; the second group $(\mathrm{n}=7)$ consisted of overweight participants $(B M 1$ $25.0-29.9 \mathrm{~kg} / \mathrm{m} 2)$ and the third group $(\mathrm{n}=15)$ consisted of obese participants $(\mathrm{BMI} \geq 30.0 \mathrm{~kg} / \mathrm{m} 2)$. Participants had to wear the armband for 7 consecutive days and fill out the following 4 questionnaires: 1) WHO Quality of life questionnaire; 2) Health at work survey; 3) International physical activity questionnaire; 4) Food frequency table. Furthermore, participants had to log their daily food consumption in a food log book. After the 7 days the data collected was analyzed using an ANOVA and descriptive methods. Results showed significant differences in calorie expenditure ( $p=0.033)$, activity patterns $(p=0.017)$, amount of steps walked per day $(p=0.018)$ and the amount of hours slept per night $(p=0.45)$ between the three groups. The overweight group had higher energy expenditure, activity patterns, and the number of steps per day. The normal group recorded the most hours of sleep, with obese group sleeping the least amount of hours. In conclusion, overweight and obese individuals sleep fewer hours than normal weight individuals. Whether the lack of sleep in these groups caused weight gain or was the result of being obese or overweight could not be determined. The overweight group was more active and had higher energy expenditure than the obese group as hypothesized, but these values were also higher than the normal group. This could have been caused by only considering BMI for classification, leaving out lean muscle mass as a contributing factor to increased weight.

Key Words: Lifestyle habits, sleeping patterns, physical activity patterns, BodyMediaßFITArmband.

Muhammad Dawood

Affiliation: University of Pretoria

Additional Authors: Muhammad Dawood, Agatha Johanna Van Rooijen, Piet J Becker, Anna Maria Marais

\section{The inter- and intra-rater reliability of a technique for assessing the length of the Latissimus Dorsi muscle}

Background: Evidence-based practice requires the use of objective, valid and reliable tests for measuring the length of a muscle. No evidence of reliability for any technique testing the length of Latissimus Dorsi (LD) was found.

Purpose: To assess the inter-rater and intra-rater reliability of a technique adapted by Comerford and Mottram (2012) for assessing the length of LD. Methods: Fifty-six students from the University of Pretoria's Physiotherapy Department were the participants in this study. Four physiotherapists with varying numbers of years of clinical experience independently performed the test for assessing the length of LD. The test was performed twice by each physiotherapist on every participant and two measurement sessions took place. A pilot study wascarried out to test and time the procedure. The intra-class correlation coefficient(ICC) as determined in a mixed-effects GLS regression analysis was used to assess the inter and intra rater reliability of the LD length test. A 0.05 level of significance was employed.

Major Results: A sample of 56 participants provided an intraclass correlation coefficient (ICC) that varied between 0.55 and 0.76 and this is regarded as moderate to poor reliability.. The ICC between the experienced raters was found to be 0.48 , with a novice rater having an ICC of 0.48 as well. The ICC between all the raters was 0.33 .

Conclusion: The poor reliability of the technique testing the length of LD was identified and addressed in order for adequate usage thereof, in research and in practice. Recommendations were made.

Key words: Latissimus Dorsi, muscle length test, dysfunction; reliability; glenohumeral joint 


\section{Hip and Groin Pain in Sub Elite South African Footballers}

Introduction: Groin injuries are common in football players, attributed largely to the nature of the sport involving rapid accelerations, decelerations, abrupt directional changes and kicking. It has been suggested that up to a third of players will sustain a groin injury in their careers and this may mean months off from the field, and predispose them to further injuries. Previous groin injuries are a well-identified risk factor for sustaining future groin injuries, suggesting that players are inadequately rehabilitated or that the original predisposing risk factors have not been addressed.

Objectives: The study aims to describe the prevalence, nature and treatment patterns of groin injuries in sub-elite players, and to investigate differences in hip strength and range of motion between players with and without a history of groin injury.

Method: Thirty sub-elite male players were interviewed and then assessed using the Copenhagen Hip and Groin Outcome Score (HAGOS) questionnaire, isokinetic hip flexion/extension strength, adductor squeeze test and range of motion.

Results: Seventeen players (57\%) reported a previous groin injury, of whom five $(29 \%)$ stated that they had not soughttreatment from a medical professional. Of the players who consulted a sports physician (6\%) or physiotherapist $(65 \%)$, the diagnoses included adductor strain (35\%), inguinal rupture $(18 \%)$, iliopsoas muscle injury $(12 \%)$, hip joint pathology $(6 \%)$ and chronic groin pain $(6 \%)$. The average time before full return to play was 25 days. Previously injured players had a significantly lower HAGOS score (83.4 \pm 15.9$)$ than the non-injured players $(92.0 \pm 5.2)$. No significant differences in hip flexion/extension isokinetic strength, hip range of motion and adductor strength were identified between the two groups.

Conclusion: The prevalence of groin injuries in this population is very high and requires lengthy rehabilitation time. Only two-thirds of players consulted a medical practitioner with their injuries, increasing the likelihood that rehabilitation was inadequate. Improved player and coach education regarding injury management may be required for better rehabilitation, in order to reduce subsequent injuries.

Adiele Dube

Affiliation: Sports Scientist

\section{Blood Pressure among Zimbabwean urban primary school children aged 5 to 11 years.}

Background: Following few publications available with data on blood pressure profiles of Zimbabwean population, especially children and adolescents, few data exist on urban and rural school going children. The aims of this study were to examine the incidence of hypertension (HT) among Zimbabwean urban chZimbabwean urban children residing in Kwekwe; and to determine the relationship between body mass index (BMI) and blood pressure (BP) among them. The study involved 480 primary school children (230 boys and 250 girls) aged 5-11 years.

Methodology: Stature and body weight were measured using standard procedures. BMI for gender and age defined overweight. BP was monitored for thrice consecutively using validated electronic devices (Omron 7051T). HT was determined as the average of three separate BP readings where the systolic or diastolic blood pressure was $\geq 95$ th percentile for age and sex.

Results: The incidence of overweight among the girls (3.6\%) was higher compared with the boys (2.7\%). Both systolic and diastolic pressures (SBP and DBP) increase with age in both sexes. Potential development of hypertension among the children is noticeable at Early Childhood Development (ECD) level for both boys and girls; $1.8 \%$ and $1.5 \%$ respectively and ranged from $0.8 \%$ to $1.8 \%$ for boys and $2.0 \%$ to $5.3 \%$ for girls. The overall incidence of hypertension was $1.5 \%$ and $2.6 \%$ in boys and girls, respectively. The highest noticeable value for boys was at ECD and decreased with increase in grade level. The incidence of hypertension (SBP > 95th percentile) was $0.4 \%$ and $0.2 \%$ in boys and girls, respectively. For the girls there was a progressive increase in the tendency towards development of hypertension from ECD to 6th grade except a small d ecrease in 3rd and 4th grade levels. Girls in the 6th grade level showed the highest value of incidence of hypertension (5.3\%). The blood pressures (SBP and DBP) significantly correlated with age, stature, body mass and BMI $(P<0.05)$.

Conclusion: The findings demonstrate that elevated blood pressure is prevalent among urban Zimbabwean children and that there is need for routine measurement of BP to children residing in this region as part of physical examination for physical activity in schools. The use of BMI cutoffs tailored to metabolic risks may be vital for assessment of overweight. BP increased with age in both sexes, and this significantly correlated with age, stature, body weight and BMI. Overweight, Body mass index, Blood pressure, Mining industry, Urban children, Zimbabwe

Sean Finaughty

Affiliation: University of Pretoria

Additional Authors: Sean Finaughty, Hannah van Buuren, Jamie Schultz

\section{Changes in Hydration Status, Cognition and Movement Time in Male Cricket Players during three consecutive days of Match play}

Introduction: Changes in hydration have been shown to negatively affect performance. In cricket, there are increasingly more matches with greater intensities placing greater demands on players. This along with increased exposure to higher temperatures and humidity, increasing time spent in the field wearing protective equipment and clothing which are not effective for sweat evaporation may lead players to extreme thermal stress. This study examined changes in hydration status, cognition and reaction to an audio and visual stimulus, during the fielding innings' of three day cricket in male

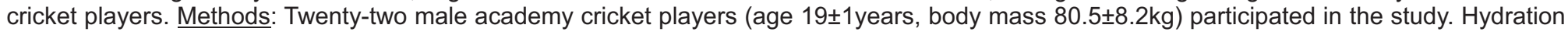
status measures included body mass (BM), a subjective thirst rating (TR) and urine osmolality (Uosm). Cognition (Letter Digit Substitution Test LDST) and audio and visual reaction timing test were also examined. Daily measurements were recorded on arrival prior to breakfast (baseline) and at the start and end of each fielding innings (pre- and post-innings). Results: Statistical differences observed between daily baseline measures were inconsistent for both baseline and pre-and post-innings measurements across the three days. Mean decrements in BM without correction for fluid and food intake ranged from 1.8 to $3.7 \%$, while values with correction for fluid and food intake produced significant net- innings decreases in BM of 0.36 $0.78 \%(p<0.05)$. Increased TR and decreased Uosm trends associated with decrease in BM were observed but no statistical significance was found. LDST and audio-visual reaction timing indicated no significant or consistent changes across the five innings' studied. CONCLUSION: The potential for dehydration $(>2 \%$ decrease in body mass, Uosm $>900 \mathrm{mOsm} / \mathrm{L}$ and high TR) during the fielding innings is a reasonable, viable concern for cricketers. However, in this study, fluid and food consumption practices were sufficient to limit any potentially deleterious effects of dehydration on cognition and reaction timing. 


\section{Mental Toughness of Rugby Players during Periodization Phases}

Information on the contribution of psychological skills and mental toughness on performance is still limited within the sporting context, even though it has been proven that these factors influence sporting achievement. Similarly, no agreement on how to develop and improve mental toughness during training programs has been achieved. The aim of this study was to explore the differences in mental toughness of rugby players during periodization phases, as well as the differences in mental toughness between rugby 'forwards' and 'backs'.

Data from rugby players, who participate in the sport on a university and regional level, was obtained by means of the Psychological Performance Inventory; that the inventory was completed by all players during the preparatory, competition, and transition periodization phases. Differences in mental toughness were analysed using a repeated measures mixed linear model with periodization phase, team and group (forwards versus backs) as fixed effects, and fitting an unstructured covariance matrix to the repeated measures of mental toughness. From this model, mean values for each group and phase, as well as differences between mean values between periodization phases together with associated $p$-values and $95 \%$ confidence intervals were calculated. The results indicated that there are significant differences amongst rugby players' attitude control ( $p=0.00)$, namely significantly higher values during competition phase than during the preparation phase. When the components of mental toughness were compared for the competition phase and the transition phase, significant differences were found for overall mental toughness $(p=0.01)$, motivation ( $p=0.04)$, and attitude control $(p=0.05)$, with the latter phase scoring lower than the competition phase. No significant differences were found between the mental toughness components of the preparation phase compared to the transition phase. The mental toughness of forwards and backs was not significantly different, although there was a tendency for backs to have higher mean values for all of the mental toughness components. This study was designed to explore mental toughness of rugby players during periodization phases, and could be the foundation for future studies to further investigate which mental toughness components seems to be dominant during specific periodization phases - and which components still have room for improvement in order to facilitate the development of training guidelines that enhances athletes' performances and help them to achieve their goals.

Keywords: Mental toughness, Rugby, Periodization phases. 
Chrisna Janse van Rensburg

Affiliation: University of the Free State

Additional Authors: Riaan Schoeman, Frederik Coetzee

\section{Positional tackle and collision rates in Super Rugby}

The aim of this study was to evaluate differences between playing positions with respect to tackle and collision rates in a rugby game, and to determine the collision rates of individual playing positions in professional rugby union. Data from 30 matches (both teams involved) during the 2013 Super Rugby Season were captured and supplied by the Cheetahs Super Rugby Franchise using the Verusco TryMaker Pro. The results show that there were significant differences between positional groups regarding the tackle rates; however the front row players and the second row players did not differ significantly (positions 1,2 , or 3 vs positions 4 ,or $5 ; p=0.0715$ to $p=0.6324$ ). Within a positional group, the difference between the inside centre and the other inside backs was significant ( 9 vs $12 ; p=0.0029,10$ vs $12 ; p=0.0045$ and 12 vs $13 ; p=0.0100$ ). Furthermore, there were no significant differences between second row players and the eigthman ( 4 vs $8 ; p=0.4183$ and 5 vs $8 ; p=0.6863$ ) although significant differences were found between second row players and the rest of the loose forwards $(6$ and 7$)$. A significant difference $(p<0.05)$ exists between all forwards and backs when collision rates are compared. In summary, this study revealed significant inter-positional differences in tackling and collision rates within the same positional group.

Keywords: Rugby, Tackle, Collisions, Playing positions, Rucking.

Chrisna Janse van Rensburg

Affiliation: University of the Free State

Additional Authors: Riaan Schoeman, Frederik Coetzee

\section{Kicking statistics that discriminate between winning and losing teams in the 2013 Super Rugby season}

Kicking is one of the fundamental movements associated with rugby union and the successful completion of this task may lead to team success. Furthermore, kicks are an aspect of the game which has seen noticeable changes. The current trend in video analysis is the development of performance profiles to describe individual or team patterns created from a combination of key performance indicators. However, despite the range of detailed analyses there is no obvious structure or progressive evolution to the development of analysis methods and there are still large gaps in the literature, especially in the area of rugby. Previous research indicated that losing teams were more likely to try to control the ball through accurate passing, where winning teams had a more kick-related game plan. In this study, a total of 900 professional rugby players that participated in 30 games played during the 2013 Super Rugby competition were observed. Two games from each of the participating franchises were used. The aim of the study was to discriminate between the kicking statistics of winning and losing teams in the 2013 Super Rugby season. Previous research found that kicking the ball away and making more tackles than the opposition were the two most influential factors in determining winning from losing. Results from the present study suggest that winning teams tend to kick the ball more (average of 25.77 kicks per game) than losing teams (20.23). Losing teams gain a mean total of $660.0 \mathrm{~m}$ per game in comparison to winning teams, $901.4 \mathrm{~m}$ per game. Thus the study showed that winning teams kick more, with greater distance. Winning teams also have greater kick frequency when attacking minutes of play are considered. More research is required to account for weather, importance of the game and match venues. Good playing conditions were associated with large increases in tries and points scored, and with large decreases in kicks in play and participation time per player.

Keywords: Kicking, Performance indicators, Metres gained.

Chrisna Janse van Rensburg

Affiliation: University of the Free State

Additional Authors: Riaan Schoeman, Frederik Coetzee

\section{Passing statistics that discriminate between winning and losing teams in the 2013 Super rugby season.}

Passing is a common performance indicator in rugby since this action is performed by all players. Evaluating the indicators such as number of kicks, rucks, mauls, passes, and tries can provide valuable insight into team performance and may be used to predict the future performance of rugby union teams. Increases in passes, tackles, rucks, tries, and ball-in-play time were associated with the advent of professionalism Therefore, this study evaluated the number of passes during the 2013 Super rugby season and to determine if the number of passes discriminated between winning and losing teams. Data was gathered from 30 games during the 2013 Super Rugby season using the Verusco TryMaker Pro. Data collected were statistically analysed to show the number of kicks made during a season of Super Rugby. The results of this study suggest that losing teams tend to pass the ball more (157.41) than winning teams (127.02). In agreement with these results, winning teams have higher counts of kicks. The study shows a significant difference between winning teams and losing teams regarding total passes, bad passes, and good passes $(p=<0.05)$. It can be concluded that factors such as the individual playing position, skill level of players, weather and game plan can determine the outcome of passing as a variable. Different teams use a variety of game plans and can influence the number of passes or kicks per match, and so also the outcome of the game.

Keywords: Passes, Performance indicators, Good passes, Bad passes

Barry Gerber

Affiliation: North-west University

\section{The Status of Fundamental Movement Skills of 3-Year Boys and Girls}

The period 3 to 5 years is a crucial time in the motor development of a child during which maturation constantly occurs. Contradictory results are found in literature regarding the current status of fundamental movement skills of 3 year old children and possible gender differences in this time period. The aim of this study was to determine the status of the fundamental movement skills and possible gender differences of 3 year old children by using the Kinderkinetics preschool assessment (Pienaar et al., 2013). A cross-sectional research design was conducted on 51 children (19 girls, 32 boys) with a chronological age of 3.0-3.11 years from 3 selected nurseries in the Potchefstroom and Jankempdorp areas. For descriptive purposes the data was analyzed by means, standard deviations, minimum and maximum values. An independent T-test (possible gender differences) and effect size were used for statistical- $(p \leq 0.05)$ and practical $(d \geq 0.8)$ significance. Statistical significance were found where girls outperformed boys in hopping on one leg (right) and identifying body parts and boys outperformed girls in standing on one leg (left), kicking a moving ball and copying hand signals. Improvements were found in some skills while other skills correspond with current literature. These results will contribute to more accurate identification of possible delays in early years of development. 


\section{Electrocardiographic patterns in African university strength and endurance athletes of Zulu descent}

Aim: There is concern over the effect of training on heart function of athletes as recorded by 12-lead electrocardiography (ECG). Although ECG abnormalities with respect to ethnic origin of black athletes from the Caribbean, West Africa and East Africa have been reported, black athletes from southern Africa, specifically participating in different sports, have never been investigated before. The purpose of this study was to analyse the ECG patterns in South African students of Zulu descent, who represented our university in boxing (endurance modality) and body building (resistance modality) at a regional level.

Methods: Fifteen subjects each were assigned to an endurance $(E)$, resistance $(R)$ or control $(C)$ group, respectively. ECG patterns were recorded with a 12-lead ECG.

Results: Our subjects indicated no significant differences in ECG patterns in relation to whether they participate in strength or endurance related sport. However, $80 \%$ of the endurance group and $67 \%$ of the resistance displayed ECG criteria indicative of left ventricular hypertrophy (LVH), group $E$ displays higher $R 5 / S 1$-wave voltages $(E=43.3 \mathrm{~mm} ; R=36.8 \mathrm{~mm} ; C=37.1 \mathrm{~mm})$ as well distinctly abnormal $E C G$ patterns $(E=87 \% ; R=73 \% ; C=$ $53 \%$ ), raising clinical suspicion of structural heart disease. Our cohort presented with non-significant, marked ST-segment elevation (53\% of both the E and R groups) and inverted T-waves in $27 \%$ of the E group.

Conclusion: Similar to findings in other ethnic Africans, a large proportion of our Zulu study population displayed ECG criteria indicative of LVH on the evidence of a marked increase of R5/S1-wave voltage and ST/T-segment changes with no differences in relation to whether they participate in strength or endurance related sport.

\section{Grant}

Affiliation: Section Sports Medicine, University of Pretoria

Additional Authors: CC Grant, DJ Dowson, Helen Bayne

\section{Groin Injuries in South African Elite Football Players}

Introduction/Aim: A previous groin injury is a known risk factor for sustaining a future injury. The aim of this study was to investigate differences in hip strength, hip range of motion and agility between players with and without a history of groin injury. Additional data was also used to create and overall impression of prevalence, time off from play, injuries sustained and treatment received.

Method: Thirty elite male football players were tested using the HAGOS (Hip and Groin Outcome Score) questionnaire, isokinetic hip flexion/extension strength, adductor squeeze test, range of motion and an agility test.

Results: Seventeen players (56.7\%) gave a history of a previous groin injury and thirteen players were part of the control group. The study confirmed the HAGOS as a reliable measure of hip/groin disability outcome and injury status. There were no significant differences in hip flexion/extension isokinetic strength, hip range of motion, adductor strength and agility between the two groups. $35.3 \%$ of players sustained an adductor strain. $29.4 \%$ of players did not seek treatment from a qualified professional i.e. no specific diagnosis made. $23.5 \%$ sustained an inguinal rupture, $5.9 \%$ sustained a hip joint injury and $5.9 \%$ had chronic groin pain. Only $5.9 \%$ of players consulted a sports physician, $64.7 \%$ of players consulted a physiotherapist. The average time off from play was 25 days.

Conclusion: The prevalence of groin injuries in elite football is very high, with lengthy off field time. A large proportion of players (29.4\%) did not consult a qualified practitioner with their injuries resulting in inadequate rehabilitation. Perhaps the best way to avoid future injuries is educating players/coaches to seek correct professional help.

\section{Grant}

Affiliation: Section Sports Medicine, Faculty of Health Science, University of Pretoria

Additional Authors: CC Grant, A Jansen van Rensburg

\section{Evidence-Based Prescription for Cyclo-Oxygenase-2 Inhibitors in Sports Injuries}

Introduction: Healthcare professionals are increasingly under pressure to return athletes to play in the shortest possible time. There is limited choice in providing treatment that speeds up tissue repair, while simultaneously maintaining good quality of healing. Inflammation forms a fundamental part in the process of tissue repair.

Discussion: However, excessive inflammation may cause more pain, and limit functional restoration. Although the use of anti-inflammatory treatment in the form of a cyclo-oxygenase-2 inhibitor (coxibs) has been widely recognised as being effective, the potential detrimental effect on tissue repair, as described mainly in animal model studies, needs to be taken into account. The side-effects profile on the gastrointestinal tract favour coxibs over non-traditional NSAIDs. The possible effects on the renal and cardiovascular systems also need to be considered. The prescription of coxibs should be pathology and situation specific.

Conclusion: There are no clear guidelines on the correct time of administration and the duration of the course, but it seems that the literature is in agreement that they should be administered for a limited time at the lowest effective dose possible. 


\section{Grant}

Affiliation: Section Sports Medicine, University of Pretoria

Additional Authors: E Minnaar, CC Grant

\section{Physical Activity of Children From A Rural Town, South Africa}

Introduction/Aim: Low- and middle-income countries, including South Africa, are witnessing the fastest rise in overweight children. The main reason identified is inactivity. The study aim was to determine the physical activity measured in boys and girls, from three different age groups, living in rural South Africa. The influence of BMI, gender and age on activity measurements was also investigated.

Methods: Seventy-eight rural children, divided in three groups according to their age, were issued a pedometer for seven days. The Mann-Whitney 2-tailed test was used to assess gender differences. Linear regression analyses were performed to determine the role of the predictors (BMI, gender and age) on the pedometer-measured dependent variables.

Results: Between 12-14 years boys gave $18.2 \%$ more steps than girls $(p=0.042)$. Boys between 9 and 11 years gave $26.5 \%$ more steps than girls $(p=0.003$ ), and $5-6$ year old boys (although not statistically significant; $p=0.481$ ), gave $11.9 \%$ more steps per day than girls of the same age. The regression model including $B M I$, gender and age can explain moderate to large portions of variance in average total steps $(R 2=0.119 ; p=0.024)$, calories $(R 2=0.402 ; p<0.001)$, distance $(R 2=0.333 ; p<0.001)$ and fat burned $(R 2=0.398 ; p<0.001)$, but not in aerobic steps count $(p=0.153)$ and aerobic walk time $(p=0.135)$.

Discussion/Conclusion: Older boys were more active than the older girls and physical activity levels differ between different age groups. Activity increased for both boys and girls from the age between 5 and 6 , to the age between 9 and 11. However, this was followed by a significant decrease in physical activity for ages between 12 and 14 . Gender and age influences the activity of children. The age group 9 to 11 may be the ideal time to focus gender specific intervention programs to prevent the significant decrease in activity during the ages of 12 to 14 and to promote a lifestyle of high physical activity.

\section{Andrew Green}

Affiliation: Biomechanics Laboratory, School of Physiology, University of the Witwatersrand, Johannesburg

Additional Authors: Andrew Green, Chloe Dafkin, Benita Olivier, Samantha Kerr, Warrick McKinon

\section{The trade-off between distance and accuracy in the Rugby Union place kick}

Kicking is a ballistic movement that requires controlled and intricate coordination between the segments of the lower body, both temporally and spatially thereby making it a complex biomechanical action. Little attention has been given to the rugby union place kick, especially in the outdoor setting. The kicker must have the ability to kick the ball long distances as well as be accurate enough to get the ball through the up-rights, spaced $5.6 \mathrm{~m}$ apart. The success of a place kick is currently thought to be reliant on both physiological factors such as balance and strength and on biomechanical skill, such as the optimization of the kinetic chain. The study aim was to test the relationships between distance, accuracy and the kinematics of place kicking performance of rugby players in an outdoor setting. Twelve first team university rugby players (age $22 \pm 3$ years, mass $88.73 \pm 12.38 \mathrm{~kg}$, stature $179 \pm 6 \mathrm{~cm}$, playing experience $11 \pm 4$ years) had fully body kinematics measured for five place kicks. All kinematics were recorded at $100 \mathrm{~Hz}$ using an 18 camera system and all biomechanical variables were calculated using custom written algorithms. Kick distance and accuracy were directly measured. The kick distance was defined as the length from the midpoint on the half way line to the landing position of the ball. Accuracy was determined as the width error spread (standard deviation of the participants' kicks) between the landing position of the ball and the centre line. The average kick distance achieved was $45.35 \pm 5.27 \mathrm{~m}$, with an average accuracy spread of $5.81 \pm 3.47 \mathrm{~m} 2$. The current study showed a positive correlation between shoulder $(r=0.755)$ and pelvis $(r=0.661)$ rotation, and kick distance. Further positive correlations between and playing experience $(r=0.596)$ were related to the accuracy of the kick. Negative correlation between stance elbow flexion ( $r=-0.780)$, shoulder rotation $(r=-0.744)$ and $X$-factor $(r=-0.785)$ were noted for kick accuracy. Place kick distance could potentially be maximized by improving shoulder and pelvic rotations and place kick accuracy could be improved by full extension of the stance arm. Our data suggests that larger shoulder rotations may promote kick distance while impeding kicking accuracy. 
Henriette Hammill

Affiliation: University of Zululand

Additional Authors: Henriette Hammill, Nicolene Smith, S'fiso Thabethe

\section{The effects of acute self myofascial release (MFR) and stretching techniques on physical fitness parameters}

Measurements were taken initially and then participants were randomly divided into four groups (control, static stretching, dynamic stretching and self MFR). During the intervention programme the various groups took part in prescribed stretching techniques under supervision of qualified technicians for an hour. Baseline measurements were repeated, except for BMI and fat percentage. Repeated measures using ANOVA revealed significant differences with a number of variables: hip flexor flexibility for both legs (HFFR; $p=0,043 ; H F F L ; p=0,002)$, sit ups (SU; $p=0,002)$ and deep squat (DS; $p=0,006)$. ANCOVA measures revealed that there was significant improvement in sit ups $(S U ; p=0,014)$ and deep squats $(D S ; p=0,025)$ in the dynamic group, compared to the control group. In conclusion, both MFR and dynamic stretches are beneficial in improving acute selected flexibility and strength parameters. Dynamic stretches show a more significant improvement compared to MFR in selected flexibility and strength parameters. This information is valuable to sport participants, providing information on the ideal stretching techniques to administer before events, which can lead to an enhancement in performance and reduction in the prevalence of injuries related to partaking in physical activity by means of increased flexibility and/or ROM. The objective of this study is to assess the effects of self myofascial release (MFR) techniques and different stretching techniques on physical fitness parameters on thirty (30) university students who participate in team sports. The design used in this research project was a prospective randomized controlled study where each participant was given a numeric participation code. Baseline measurements were taken initially and then participants were randomly divided into four groups (control, static stretching, dynamic stretching and self MFR). During the intervention programme the various groups took part in prescribed stretching techniques under supervision of qualified technicians for an hour. Baseline measurements were repeated, except for BMI and fat percentage. Repeated measures using ANOVA revealed significanrevealed significant differences with a number of variables: hip flexor flexibility for both legs $(H F p=0,002)$, sit ups (SU; $p=0,002)$ and deep squat (DS; $p=0,006)$. ANCOVA measures revealed that there was significant improvement in sit ups $(S U ; p=0,014)$ and deep squats $(D S ; p=0,025)$ in the dynamic group, compared to the control group. In conclusion, both MFR and dynamic stretches are beneficial in improving acute selected flexibility and strength parameters. Dynamic stretches show a more significant improvement compared to MFR in selected flexibility and strength parameters. This information is valuable to sport participants, providing information on the ideal stretching techniques to administer before events, which can lead to an enhancement in performance and reduction in the prevalence of injuries related to partaking in physical activity by means of increased flexibility and/or ROMThe objective of this study is to assess the effects of self myofascial release (MFR) techniques and different stretching techniques on physical fitness parameters on thirty (30) university students who participate in team sports. The design used in this research project was a prospective randomized controlled study where each participant was given a numeric participation code. Baseline measurements were taken initially and then participants were randomly divided into four groups (control, static stretching, dynamic stretching and self MFR). During the intervention programme the various groups took part in prescribed stretching techniques under supervision of qualified technicians for an hour. Baseline measurements were repeated, except for BMI and fat percentage. Repeated measures using ANOVA revealed significant differences with a number of variables: hip flexor flexibility for both legs (HFFR; $p=0,043 ; \mathrm{HFFL} ; p=0,002)$, sit ups (SU; $p=0,002)$ and deep squat (DS; $p=0,006)$. ANCOVA measures revealed that there was significant improvement in sit ups $(S U ; p=0,014)$ and deep squats $(D S ; p=0,025)$ in the dynamic group, compared to the control group. In conclusion, both MFR and dynamic stretches are beneficial in improving acute selected flexibility and strength parameters. Dynamic stretches show a more significant improvement compared to MFR in selected flexibility and strength parameters. This information is valuable to sport participants, providing information on the ideal stretching techniques to administer before events, which can lead to an enhancement in performance and reduction in the prevalence of injuries related to partaking in physical activity by means of increased flexibility and/or ROM

\section{Ernest Hobbs}

Affiliation: High Performance Centre, University of Pretoria

Additional Authors: Ernest Hobbs, Helen Bayne

\section{Foot and Ankle Kinematics in Male and Female Adolecent Endurance Runners}

Introduction: Research has provided a wealth of information regarding lower leg kinematics in adult endurance runners [1, 2, 3]. The aim of the current study was to investigate foot and ankle kinematics in adolescent endurance runners, and to investigate differences between males and females.

Methods: Twenty endurance runners (6 males, 14 females), aged $15-18$ years, undertook a 5 minute run at approximately $90 \%$ of their training pace, at either $10.5 \mathrm{~km} / \mathrm{h}$ (females) or $12 \mathrm{~km} / \mathrm{h}$ (males) on a treadmill set to $0 \%$ incline. The final 20 seconds of each run was filmed at $120 \mathrm{~Hz}$ from both the left and right side, and $60 \mathrm{~Hz}$ from the rear. Ankle dorsiflexion was measured at impact, as the angle between a line from the head of the fibula to the lateral malleolus, and the plantar surface of the outsole of the shoe. Rearfoot eversion was measured at mid-stance, as the angle between the posterior midline of the lower leg and the posterior midline of the calcaneus.

Results: Analysis of lower leg running kinematics found that rearfoot eversion was similar between males $\left(7.0^{\circ} \pm 1.6\right.$ and $9.2^{\circ} \pm 2.8$ for left and right respectively) and females $\left(8.5^{\circ} \pm 2.9\right.$ and $8.6^{\circ} \pm 2.9$ for left and right respectively), however ankle dorsiflexion showed statistically significant differences for the right foot (males: $92.0^{\circ} \pm 5.8$, females: $85.1^{\circ} \pm 4.2, t=3.02, p<0.01$ ) and near significant differences for the left foot (males: $89.9^{\circ} \pm$ 5.4 , females: $86.0^{\circ} \pm 3.5, t=1.96, p=0.065$ ).

Discussion: Rearfoot eversion angles were comparable with norms from literature $[1,2,3]$. The female athletes in the current study tended to make contact in a more dorsiflexed position, which is associated with a more pronounced heel strike. This may have clinical relevance, as impact forces and injury patterns differ between footstrike patterns [4]. The influence of absolute running velocity on ankle kinematics must also be considered, and it is recommended that future studies examine changes in kinematics at varying velocities in this population. 
Erik Hohmann

Affiliation: Musculoskeletal Research Unit, CQ University, Australia Medical School University of Queensland, Australia Additional Authors: Erik Hohmann, Tony Young, Ross Clark, Adam Bryant, Peter Reaburn

\section{Standing balance is not an important predictor of function in ACL-deficient subjects - a case control study}

Introduction: Previous research has demonstrated significant proprioceptive deficits including balance in ACL-deficient knees. The purpose of this project was to investigate the use of standing balance as an indirect measure of proprioception and to investigate the relationships between balance, strength, hopping tests and subjective functional outcome scores.

Methods: For this project the Nintendo Wii Balance Board (NWBB) was used as a validated tool for measuring balance. Twenty-seven ACL-deficient subjects with a mean age of 28+9.6 (Range 15 to 50) and thirty-eight control subjects with a mean age of $26.7+6.7$ (Range 15 to 57 ) were tested. Single leg standing balance, isometric quadriceps strength, and isometric hamstrings strength tests were performed using the NWBB and customwritten software. All subjects performed single leg hops and vertical jumps, and completed Lysholm and IKDC questionnaires.

Results: Statistical analysis comparing control and ACL-deficient groups revealed significant between group differences for standing balance $(p=0.02)$, quadriceps strength $(p=0.006)$, hop $(p<0.0001)$, vertical jump $(p=0.02)$, and Lysholm score $(p<0.0001)$. However multivariate analysis within the ACL-deficient group demonstrated no significant relationships between variables.

Conclusions: The results of this study suggest that while there are significant differences between the healthy ACL-intact subjects and the ACLdeficient group in all variables, standing balance is not an important predictor of subjective function in the ACL-deficient group.

Erik Hohmann

Affiliation: Musculoskeletal Research Unit, CQ University, Australia Medical School University of Queensland, Australia

Additional Authors: Erik Hohmann, Kevin Tetsworth

\section{Large Osteochondral Lesions of the Femoral Condyles: Treatment with Fresh Frozen and Irradiated Allograft using the Mega OATS Technique}

Purpose: Fresh and fresh frozen allograft has been used by many surgeons as a viable and effective treatment for large osteochondral lesions of the femoral condyles. Irradiated allograft transplantation may be an alternative option and result in similar clinical outcome. The purpose of this study was to review the clinical results of irradiated fresh frozen osteochondral allografts for large osteochondral defects of the knee using the Mega-OATS technique.

Methods: A total of nine patients (5 males, 4 females) with a mean age of 32.1+6.6 (18-44) underwent Mega-OATS transplantation with irradiated (2.5Mrad), fresh frozen distal femur allograft. Three patients underwent ACL-reconstruction; one patient a high tibial osteotomy. The underlying cause was osteochondrosis dissecans in four and trauma in five patients. The defect size was $25 \times 25 \mathrm{~mm}$ in three patients and $30 \times 30$ in six patients. All OCD lesions were located on the medial femoral condyle; two of the traumatic lesions were located on the lateral femoral condyle. Clinical outcome was assessed by using the Lysholm and IKDC scores. Radiographic incorporation was evaluated using serial radiographs and MR imaging at one year post surgery. All patients were reviewed at 3,6,12, and 24 months following surgery.

Results: The Lysholm score improved significantly $(F=69.9, p=0.02)$ within and between patients during the follow-up period from 40.9 to 90.9 at 2 years. The IKDC score also improved significantly $(F=118, p=0.007)$ within and between patients during the follow-up period from 37 to 87.1 at 2 years. Radiographic union was observed in all patients at three months; on MR imaging at one year osseous integration was observed in 8 patients. Graft subsidence with loss of the overlying cartilage was observed in one and subchondral cystic changes at the implantation side was seen on another patient.

Conclusion: The results of this small case series suggest that irradiated osteochondral allograft provides significant medium-term clinical improvement in patients treated for large osteochondral lesions of the femoral condyles.

\section{Erik Hohmann}

Affiliation: Musculoskeletal Research Unit, CQ University, Australia Medical School University of Queensland, Australia Additional Authors: Erik Hohmann, Cariad Wratten, Kevin Tetsworth

\section{Comparison of Three-Dimensional Between Gender Femoral Notch Volume in ACL-deficient versus ACL-intact Subjects}

Purpose: The purpose of this study was to evaluate between-gender differences in three-dimensional femoral notch volume in anterior cruciate ligament $(\mathrm{ACL})$-deficient patients compared to a matched control group of $\mathrm{ACL}$-intact patients.

Methods: Magnetic resonance images from 90 ACL-deficient subjects (45 males, 45 females) aged 16-50 years were identified from our departmental database, and then compared to a matched control group of ACL-intact subjects. The three-dimensional femoral notch volume on each MRI was measured using software specific tools. One-way ANOVA was used to compare notch volume between ACL-deficient and ACL-intact groups and between the two genders. The relationships between height, weight, BMI and notch volume for each group and within the male and female cohort were established using Pearson's moment correlations.

Results: Femoral notch volume in the female ACL-deficient group was $3.1 \pm 0.70 \mathrm{~cm} 3(\mathrm{Cl} \pm 0.2)$ compared with $3.6 \pm 0.7 \mathrm{~cm} 3(\mathrm{Cl} \pm 0.2)$ in the female control group. Notch volume in the male ACL-deficient group was $4.5 \pm 1.1 \mathrm{~cm} 3(\mathrm{Cl} \pm 0.3)$ compared to $5.3 \pm 1.2 \mathrm{~cm} 3(\mathrm{Cl} \pm 0.4)$ in the male control group. There were significant differences between the male ACL-deficient and ACL-intact group $(p=0.02)$, the female ACL-deficient and ACL-intact group ( $p=0.0002)$, the ACL-deficient male and female groups $(p<0.0001)$, and between the male and female ACL-intact groups $(p<0.0001)$. There were weak and non- significant relationships $(r=0.01-0.37)$ between height, weight, BMI and notch volume between groups and within the male and female cohort.

Conclusions: These results clearly demonstrate a decreased femoral notch volume as measured on three-dimensional-MRI in ACL-deficient patients. There were highly significant differences in femoral notch volume between male and female cohorts, as well as between ACL-deficient and ACL-intact subjects of both genders. 


\section{Erik Hohmann}

Affiliation: Musculoskeletal Research Unit, CQ University, Australia Medical School University of Queensland, Australia

Additional Authors: Erik Hohmann, Krishna Kumar, Ancy Thomas, Kevin Tetsworth

\section{Short-term benefit from an intra-articular steroid injection in patients with adhesive capsulitis of the shoulder treated with physiotherapy?}

Purpose: The purpose of this retrospective study was to investigate the possible short-term benefit of a single intra-articular corticosteroid injection in those patients treated with physiotherapy when compared to a group of patients undergoing physiotherapy only.

Methods: A retrospective chart review was conducted to identify eligible patients treated over a four-year period. All female patients between 40-60 years with a confirmed clinical diagnosis of idiopathic adhesive capsulitis who completed a prescribed physiotherapy program between 2006 and 2009 were considered eligible. Sixty-three patients fulfilled the inclusion criteria, but 22 were excluded because of missing data in the medical record. The remaining 41 patients comprise the study cohort; an experienced musculoskeletal physiotherapist assessed these patients both at initial presentation and at 12 weeks. Twenty patients with a mean age of 55.1 years underwent physiotherapy only (PT only) and 21 patients with a mean age of 52.4 years received a single intra-articular dose of $40 \mathrm{mg}$ methylprednisolone followed by physiotherapy. Outcome measures included the Visual Analogue Scale (VAS) and measurement of range of motion.

Results: At final Assessment (12 weeks), significant between group differences were identified for the "PT only" group for flexion ( $p=0.01)$ and abduction $(p=0.008)$. When comparing the mean change from the initial assessment, a significant between group difference was observed for abduction $(p=0.03)$.

Conclusions: The results of this study suggest that the intra-articular injection of a single dose of cortisone has no significant short-term benefit in female patients with idiopathic adhesive capsulitis managed with physiotherapy.

\section{Erik Hohmann}

Affiliation: Musculoskeletal Research Unit, CQ University, Australia Medical School University of Queensland, Australia Additional Authors: Erik Hohmann, Kevin Tetsworth, Andreas Imhoff

\section{Plantar pressures during long distance running: An investigation of 10 marathon runners.}

Purpose: The objective of this study was to record plantar pressures using an in-shoe measuring system before, during, and after a marathon run, and to investigate the effect of fatigue.

Methods: Peak and mean plantar pressures were recorded before, after, and every three km during a marathon race in ten male runners using an in-shoe plantar pressure system in both feet. Heart rate, serum lactate levels and the Borg scale were used to assess both subjective and objective fatigue.

Results: There were no significant changes over time in peak and mean plantar pressures for either the dominant or non-dominant foot for all six anatomical areas measured. There were significant between dominant and non-dominant foot peak and mean plantar pressure differences for the total foot $(p=0.0001)$, forefoot $(p=0.0001)$, midfoot $(p=0.02$ resp. $p=0.006)$, hindfoot $(p=0.0001)$, first ray $(p=0.01$ resp. $p=0.0001)$ and $M T P(p=0.05$ resp. $\mathrm{p}=0.0001$ ).

Conclusions: Long-distance runners do not demonstrate significant changes in mean or peak plantar foot pressures over the distance of a marathon race, for either the dominant or non-dominant foot. However, athletes consistently favoured their dominant extremity, applying significantly higher plantar pressures through their dominant foot over the entire marathon distance.

\section{Erik Hohmann}

Affiliation: Musculoskeletal Research Unit, CQ University, Australia Medical School University of Queensland, Australia Additional Authors: Erik Hohmann, Kevin Tetsworth, Peter Reaburn, Andreas Imhoff

\section{Runners' knowledge of their foot type: do they really know?}

Purpose: The use of correct individually-selected running shoes may reduce the incidence of running injuries. However, the runner needs to be aware of their foot anatomy to ensure the "correct" footwear is chosen. The purpose of this study was to compare the individual runner's knowledge of their arch type to the arch index derived from a static footprint.

Methods: We examined 92 recreational runners with a mean age of 35.4+11.4 (12-63) years. A questionnaire was used to investigate the knowledge of the runners about arch height and overpronation. A clinical examination was undertaken using defined criteria and the arch index was analysed using weight-bearing footprints.

Results: Forty-five runners (49\%) identified their foot arch correctly. Eighteen of the 41 flat- arched runners (44\%) identified their arch correctly. Twenty-four of the 48 normal-arched athletes (50\%) identified their arch correctly. Three subjects with a high arch identified their arch correctly. Thirty-eight runners assessed themselves as overpronators; only four $(11 \%)$ of these athletes were positively identified. Of the 34 athletes who did not categorize themselves as overpronators, four runners (12\%) had clinical overpronation.

Conclusion: The findings of this research suggest that runners possess poor knowledge of both their foot arch and dynamic pronation. 


\title{
Can we predict knee functionality of ACL deficient and ACL reconstructed patients using tibial acceleration profiles?
}

Purpose: During abrupt deceleration tasks, tibial acceleration is indicative of tibial stability and shock transmitted through the lower limb. The purpose of this study was to examine relationships between knee functionality and tibial acceleration of ACL deficient and ACL reconstructed patients during landing from a single-leg long hop.

Methods: Knee functionality was rated using the Cincinnati Knee Rating System for the involved limb of 10 chronic, functional ACL deficient patients and $27 \mathrm{ACL}$ reconstructed patient (14 using a patella tendon (PT) graft and 13 using a semitendinosus/gracilis tendon (STGT) graft). Tibial acceleration during a single-leg long hop was assessed using a uniaxial accelerometer attached to the tibial tuberosity of each patient's involved limb. Results: Pearson product moment correlations revealed a significant $(p<0.05)$ moderate negative relationship between knee functionality and time to zero tibial acceleration in the ACL deficient patients $(r=-0.58)$, indicating patients who minimised the time of positive tibial acceleration had higher levels of knee functionality. For the PT graft group, a significant moderate negative relationship $(r=-0.55)$ between knee function and time to peak tibial acceleration was found indicating that early control of peak tibial acceleration was a determining factor following reconstruction using the PT. No significant relationships were found between knee functionality and tibial acceleration for the STGT graft patients.

Conclusions: The relationships between knee functionality and tibial acceleration suggested that those patients who were better able to arrest acceleration of the tibia during an abrupt deceleration task, whether reconstructed or not, tended to display greater knee functionality.

\section{Erik Hohmann}

Affiliation: Musculoskeletal Research Unit, CQ University, Australia; School of Medicine, University of Queensland, Australia Additional Authors: Erik Hohmann, Joyce Anthony, Kevin Tetsworth

\section{The influence of the labrum on osseous version and diameter - A glenoid morphometric study using MR Arthrograms}

Introduction: Morphometric variables of the glenohumeral joint such as glenoid version and diameter have been associated with glenohumeral instability, rotator cuff tears, osteoarthritis and the success of total shoulder arthroplasty. Although widely accepted that the labrum increases the contact area between the humeral head and scapula, its influence on version is not well understood. The purpose of this study was to determine the influence of the labrum on osseous version and diameter of the glenohumeral joint.

Methods: This is a radiological, cross-sectional study of the glenoid labral and osseous version and diameter measured on MR arthrograms that were void of glenohumeral joint pathology as determined by an independent radiologist. Patients where scanned using the Siemens Magnetom Verio 3T MRI scanners and variables measured using IntelliSpace PACS Enterprise. These variables were measured by two independent investigators. Images of the first 20 patients were used to determine inter- and intra-observer reliability (ICC). ICC demonstrated excellent inter- and intra-rater reliability ( 0.956 resp. 0.938 ) resulting in these variables being measured for all patients by a single observer. Statistical analysis involved descriptive analysis of measured variables and paired student $t$-tests to determine the difference between labral and osseous variables.

Results: 100 patient arthrograms were studied with an even site $(M=49, F=51)$ and gender $(R=56, L=44)$ distribution. Patients demonstrated a mean age of 22.2 years $(S D=4.5)$, glenoid osseous version of $-5.7 \mathrm{deg}(S D=5.3)$, labral version of -10.0 deg $(S D=5.5)$, osseous diameter of $28.1 \mathrm{~mm}$ $(S D=3.3)$ and labral diameter of $31.967 \mathrm{~mm}(S D=3.175)$. Inferential analysis using paired student t-tests demonstrated that glenoid labrum significantly $(p=0.001)$ increased retroversion by 4.3 deg and diameter by $3.9 \mathrm{~mm}(p=0.001)$.

Conclusion: This study demonstrated significant increases in glenoid version and glenoid diameter. Given these findings it may be more important to create a "bump" when repairing the labrum in order to preserve or increase retroversion rather than a simple repair to preserve the contact area.

\section{Audrey Jansen van Rensburg}

Affiliation: Section Sports Medicine, University of Pretoria

Additional Authors: Audrey Jansen van Rensburg, DC Janse van Rensburg

\section{The Use of Negative Pressure Wave Treatment in Athlete Recovery}

Regeneration and recovery is vital for maximising athlete performance in competitive sport. Lower body negative pressure (LBNP) treatment originally developed for astronauts to maintain orthostatic balance, proved substantial in clinical application improving circulation in complex wounds. Intermittent vacuum therapy may enhance blood flow throughout the entire body, possibly rejuvenating and improving the impaired physical state of injured or exhausted athletes. 22 male cricket players completed a 1-hour power gym session. Randomly divided, the treatment group received three 30 minute LBNP exposure sessions over three consecutive days ( 0,24 and 48 hours); the control group received no treatment. After 14 days groups were crossed over and the trial repeated. To determine the acute therapeutic effect, participants continued their regular coaching and competition program. Heart rate and blood pressure decreased noticeably during treatment, reverting to baseline levels after treatment. Lactate concentrations decreased in both groups after exercise termination; significantly more in the treatment than control group $(0.57 \pm 0.23$ vs. $0.78 \pm 0.22$, respectively, $p<0.001)$. Creatine kinase $(C K)$ indicated no significant decrease. Athletes' personal perceived degree of regeneration rated moderately high. LBNP therapy may have a systemic effect in lowering serum lactate levels, but not CK levels. Enhanced regeneration and recovery of athletes are still unconfirmed.

\author{
Robert Jones \\ Affiliation: Rhodes University \\ Additional Authors: Robert Jones, Andrew Todd, Candice Christie
}

\section{Ethnicity: a Key risk factor for hamstring injury}

Ethnicity has been identified as a key risk factor for hamstring injuries in intermittent sports. When compared to their Caucasian counterparts, epidemiological studies indicate professional players of Black African ethnic origin to be at an increased risk of hamstring muscle strain in Australian Rules football, rugby union and soccer. Anthropometric, biomechanical and physiological differences between Caucasian and Black African players may influence muscular fatigue responses, increasing the risk of strain injuries in the hamstring musculature. While possessing similar VO2max values to Caucasians athletes, Black African runners elicit a higher fractional VO2max utilisation, a superior running economy, as well as a greater predominance of type II muscle fibers. These distinctive features limit the applicability of research on Caucasians to players of BlackAfrican ethnicity. This is further complicated by intra-ethnic variations in muscle fiber distribution among individuals from different geographical locations within Africa (North, South, East, West Africa). Muscle phenotype characteristics are thought to influence the risk of hamstring injuries. Players with a greater proportion of type II fibers are more susceptible to hamstring fatigue induced by eccentric loading during the maintenance of the intermittent activity profile. Due to a predominance of type II fibers, Black African players are suggested to be at a greater risk of hamstring strain injury as these players experience greater eccentric hamstring muscle fatigue during match-play compared to Caucasian players. In this review, these factors are explored as they have important implications when designing and implementing training and recovery programs for BlackAfrican players. 


\section{The Impact of a Multi-Ingredient Supplement on Markers of Muscle Damage and Inflammation Following Downhill Running in Female} Athletes

Exercise-induced muscle damage (EIMD) can result in reduced muscle force, increased muscle soreness, increased intramuscular proteins in the blood, and reduced performance. Single ingredient supplementation protocols with whey protein isolate, branched-chain amino acids (BCAAs), creatine, and caffeine have been used to reduce the effects of EIMD. However, little is known about the effects of a multi-ingredient supplement on the reduction of muscle damage and repair after EIMD from downhill running. Moreover, there is little known about the effects of performance supplementation on endurance trained female athletes.

Aim: The purpose of this study was to investigate the potential effects of the ingestion of a multi-ingredient supplement on markers of muscle damage and inflammation following a single 60 minute bout of downhill running (DHR) in trained female runners.

Methods: Eight female runners were matched by VO2max ( $\geq 50 \mathrm{ml}-1 . \mathrm{kg}-1$. min-1) and menstrual cycle, and randomly assigned in a double-blind manner to consume a $28 \mathrm{~g}$ multi-ingredient supplement (NO Shotgun) $(\mathrm{N}=4,28.5 \pm 6.5 \mathrm{yrs}, 1.66 \pm 0.06 \mathrm{~m}, 57.06 \pm 3 \mathrm{~kg}$ ) or $28 \mathrm{~g}$ isocaloric flavourmatched placebo (maltodextrin) $(\mathrm{N}=4,29.5 \pm 5.12 \mathrm{yrs}, 1.60 \pm 0.06 \mathrm{~m}, 56.40 \pm 4.7 \mathrm{~kg})$ daily for four weeks pre-DHR. All participants were premenopausal, and all participants were be tested as close as possible to the mid-follicular phase (7-11 days after menses) of the menstrual cycle similar to the seven day inactive (or placebo) phase of hormonal contraceptive therapy. Participants performed a DHR after 4 weeks of supplementation, at a speed equivalent to $75 \%$ VO2max at $-10 \%$ gradient. Serum Creatine Kinase (CK), Interleukin-6 (IL-6), and C-Reactive Protein (CRP) were collected prior to DHR, immediately post-, 24 hours, 48 hours, and 72 hours post-DHR, with power output, hamstring flexibility, and muscle pain perception measurements taken at the same time points.

Results: There were no significant group $x$ time differences, but there was a main effect of time for CK $(p=0.05)$, pain perception $(V A S)$ ( $p=0.068)$, lower-limb muscle pain, and maximum squat jump power $(p=0.043)$. CRP peaked at 24 hours post-DHR and IL-6 peaked immediately post-DHR, with CRP decreasing at $48 \mathrm{hrs}$ post-DHR (-5.44\% of baseline). Serum CK increased by $14.23 \%$ from baseline to 24 hours post-DHR, decreased back to baseline by 48 hours. The VAS measures showed a significant 13-fold increase from baseline to 24 hours post-DHR ( $p=0.019)$, and an $11-$ fold increase from baseline to 48 hours $(p=0.022)$.

Conclusion: Multi-ingredient supplementation did not reduce muscle damage and inflammation compared with an isocaloric placebo, although the bout of DHR did elicit changes in muscle damage and inflammatory markers in trained female runners that were returned to baseline by 72 hours.

\section{David Lee}

Affiliation: Griffith University, Griffith University Centre for Musculoskeletal Research, Menzies Health Institute Additional Authors: David Lee, David Saxby, Prof Rod Barrett, Dr Richard Newsham-West, Dr Michael Ryan

\section{Repeatability and Agreement of Digital Image Correlation (DIC) for Regional Strain Estimates of the In-Vivo Human Patellar Tendon}

Introduction: Strain is an essential measure of tendon tissue mechanics, with distribution during functional loads helping to identify damaged or pathological regions and quantifying possible functional compromise. DIC is a non-contact image processing method that applies a correlation function to features in successive ultrasound images to estimate strain under dynamic and high-load conditions within an identified region of interest. The primary aim of this study was to assess the methodology of applying DIC to estimating localised strain behaviour of the patellar tendon. Specifically this study assessed 1) between-trial and between-day repeatability; and 2) the level of agreement of the DIC estimates with an accepted manual point-to-point method.

Methods: Seven adult subjects ( 5 males, 2 females; age $=30.5+/-3.5$ y) performed a rate controlled voluntary ramped contraction to a normalised target torque over a specified rate with simultaneous collection of sagittal plane imaging of the patellar tendon and knee extensor torque using real time B-mode ultrasonography and dynamometry, respectively. Transducer position and displacement during contractions were tracked by a 3D Vicon motion capture system. Recorded ultrasound images were subsequently processed using specialized software to estimate strain at selected regions.

Results: Intraclass correlation values for between-trial and between-day strain estimates were greater than 0.86 ( $p<0.95)$. The coefficient of variation of the root-mean-square difference $(p<0.95)$ and average mean detectable change $(p<0.95)$ for DIC estimates were $1.06 \%(0.9-1.23 \%)$ and $0.19 \%(0.15-0.23 \%)$ and $0.69 \%$ and $1.69 \%$, respectively. Limits of agreement analysis reported there was a $95 \%$ likelihood that DIC values were within $\pm 1.03 \%$ of the manual method. DIC was found to overestimate reported strain by $0.28 \%$. Intraclass correlation values for betweensession transducer position $(\mathrm{p}<0.95)$ were greater than 0.86 . Mean transducer displacement during contractions was found to be $6.97 \mathrm{~mm}(+/-$ $3.69 \mathrm{~mm})$.

Conclusion: Results show DIC to be a feasible and repeatable approach for estimation of regional strain at the patellar tendon. The accuracy and repeatability of DIC did not appear to be affected by change in transducer position or rotation in this study. 
Daniel Lithwick

Affiliation: University of British Columbia, Faculty of Medicine

Additional Authors: Daniel Lithwick, Saul Isserow, Brett Heilbron, Barbara Morrison, Hamed Nazzari, Jack Taunton

\section{Screening Young Competitive Athletes for Underlying Cardiovascular Disease in British Columbia, Canada - A SportsCardiologyBC Study}

Introduction:The Sudden Cardiac Death (SCD) of a young athlete is a rare, yet tragic event. Following the publication of a 25-year study out of Italy that showed a $90 \%$ risk reduction for SCD after the implementation of a systematic screening program, international attention towards the concept of screening has increased. Implementation of screening remains a controversial issue, with the primary disparity lying in whether a 12-lead electrocardiogram (ECG) should be included in addition to a cardiovascular focused medical history and physical examination. Currently Canada has no official screening mandate. The purpose of this study is to determine the prevalence of cardiovascular diseases that can lead to SCD in a sample of young (12-35) competitive athletes in British Columbia.

Methods: 1,319 athletes were screened. 688 participants were evaluated using a resting 12-lead ECG (European Society of Cardiology recommended) and the 12-item questionnaire, inclusive of personal history, family history and physical examination, as recommended by the American Heart Association. A protocol amendment was made following the initial round of recruitment with the elimination of the physical exam and a revised questionnaire. As such, the following 631 participants recruited were screened with ECG and a questionnaire created by SportsCardiologyBC researchers and clinicians.

Results: Of the 1,319 athletes evaluated, 98 (7.4\%) required follow-up investigation. 11 cardiovascular disorders $(0.8 \%)$ were found: probable hypertrophic cardiomyopathy (HCM), myxomatous mitral valve prolapse with mild regurgitation, mild-moderate tricuspid insufficiency with pectus excavatum, supraventricular tachycardia, paroxysmal supraventricular tachycardia, 4 cases of Wolff-Parkinson-White Syndrome, Long QT Syndrome and a restrictive ventricular septal defect. 26 participants are still under investigation for the presence of disease - notable queries include atrial septal defect, HCM, arrhythmogenic right ventricular dysplasia, and premature coronary artery disease.

Conclusion: Athletes in this study have yet to be restricted from activity, however 11 cases of cardiovascular diseases were identified and investigation into several more athletes is pending. As such, screening for cardiovascular disease in this population is likely a worthy endeavour. The AHA 12-item questionnaire produced several false-positive results, prompting the research team to revise the screening protocol. The most feasible and effective screening protocol must still be determined.

Daniel Lithwick

Affiliation: University of British Columbia, Faculty of Medicine

Additional Authors: Daniel Lithwick, Saul Isserow, Brett Heilbron, Barbara Morrison, Hamed Nazzari, Jack Taunton, Michael Luong

\section{Screening Young Competitive Athletes for Underlying Cardiovascular Disease - The SportsCardiologyBC Protocol}

Introduction: SportsCardiologyBC (SCBC) has screened 1,319 young (12-35) competitive athletes across British Columbia, Canada with 12-lead electrocardiogram (ECG) (European Society of cardiology recommended), history and physical examination (American Heart Association 12-item questionnaire) in order to investigate prevalence of cardiovascular disease. Following recruitment of the initial 688 participants, the researchers found the questionnaire to be causing several false-positive results. Further, they found that the physical examination had a low utility to detect disease, and that physician time was scarce and expensive. Therefore, a new screening protocol was developed in which the physical examination was eliminated and a new questionnaire was created. The questionnaire includes positive and negative questions on symptoms in an attempt to differentiate what might be cardiac causes in the absence of a physician.

Methods: 688 participants were screened using ECG and the 12-item questionnaire from the AHA and the following 631 participants were screened using the ECG and the SCBC questionnaire. In order to determine if it may be more feasible and effective to screen large athlete populations without a physician present, the positive predictive values (PPV) of the two protocols have been calculated.

Results: Of the first 688 participants screened, $61(8.9 \%)$ required follow-up testing; of which 5 were confirmed to have cardiovascular disease, with 9 still undergoing investigation. In the following 631 participants screened using the SCBC protocol, 37 (5.9\%) required follow-up testing, of which 6 athletes were found to have cardiovascular disease, with 18 still undergoing investigation. Without consideration of those still under investigation, the initial protocol yields a PPV of $9.6 \%$, and the SCBC protocol yields a PPV of $31.6 \%$.

Conclusion: Following the experience with the first 688 participants, the research team felt that a more feasible and effective screening protocol could be developed. Based on the increased positive predictive value and lower absolute and relative number of false-positives, this was found to be correct. Concerns regarding false negatives with the elimination of the physician from the screening process are valid, and as such further studies with proper ascertainment of false-negative rates must be conducted to determine sensitivity and specificity.

Rochelle Louw

Affiliation: University of Pretoria

Additional Authors: Rochelle Louw, Licinda Pienaar

\section{The Effect of Movement Instruction on Muscle Activation}

Introduction: The primary objective of this study was to determine if various muscles could more successfully activated during a specific movement/exercise when receiving instruction("Kinetic Precision"method or technique) as opposed to no or minimal instruction. The "Kinetic Precision" method included placing the body in a favourable position and using "Conscious Initiated Muscular Resistance" (mind-body connection). Methods: A cross-sectional comparative design was used. Twenty subjects performed six movements. The subjects performed the movements without instruction and then again, after receiving specific instructions ("Kinetic precision" technique). The movements or exercises were performed while surface electromyography (EMG) measurements were taken of fourteen different muscles groups. EMG data was collected and analysed using the Noraxon, EMG \& Sensor Systems (Scottsdale, Arizona) using standard EMG techniques and protocols. The Wilcoxon Signed Rank test was used to determine if there was a statistically significant $(p \leq 0.05)$ difference between measurements.

Results: There were significant differences $(p \leq 0.05)$ in terms of muscle activation between test one and test two for ten of the fourteen muscles tested using surface EMG. Namely, Side lying Gluteus maximus exercise: M. Gluteus maximus $(p=0.001)$ and M. Erector spinae- Lumbar $(p=0.027)$; Sitting knee extension exercise: M. Rectus femoris $(p=0.00)$ and M. Erector spinae- Lumbar $(p=0.00)$; Side lying Gluteus medius exercise: M. Gluteus medius ( $p=0.004)$; Sitting Arm flexion exercise: M. Trapezius- upper $(p=0.000)$; Sitting theraband exercise: M. Infraspinatus $(p=0.040)$ and M. Trapezius- middle $(p=0.025)$; and Supine bridge exercise: M. Gluteus maximus $(p=0.000)$ and M. Biceps femoris $(p=0.033)$.

For all the significant differences, test 2 (with instruction - Kinetic Precision) was greater in terms of muscle activation except for the Sitting Arm flexion exercise: M. Trapezius - upper ( $p=0.654)$; Sitting theraband exercise: M. Trapezius - middle ( $p=0.025)$; and Supine bridge exercise: M. Biceps Femoris $(p=0.033)$.

Discussion/Conclusion: The desired effect with regards to muscle activation increasing or decreasing significantly (pË,0.05) was achieved in eight muscles. Therefore results appear favourable regarding the effects of "Kinetic Precision technique using Conscious Initiated Muscular resistance" in terms of exercise form and muscle activation. Further research is recommended using a larger sample and possibly comparing various instruction techniques using a cross-over design. 


\section{Hallux Valgus in the Female Ballet Dancer: A literature review on risk factors}

Background: There is little consensus about the aetiology of hallux valgus (HV), but certain risk factors are widely agreed upon. It has long been believed that dance, specifically the ballet technique of dancing en pointe, causes HV. Due to very little research on the matter, the biomechanics, associated risk factors, conditions and injuries in dance are poorly understood.

Methodology: The University of Johannesburg library, electronic databases, as well as general search engines, such as Google-Scholar, were searched using specific search terms. Books and articles were sifted according to their content. Resources that contained dance and $\mathrm{HV}$ were then evaluated according to key criteria: HV and dance, dancer versus non-dancers, dance biomechanics, etc. The findings obtained from the literature were then assimilated.

Discussion: Dancers are exposed to the same risk factors as the general population: age, gender, heredity, constrictive footwear and anatomical factors like hypermobility, achilles tendon tightness, pes planus, to name a few. Dancers, however, have some risk factors that are unique to them: dance itself, poor technique and, notoriously, the shoewear, specifically the pointe shoe which enables a dancer to stand and dance on the tips of her toes. This literature review found that even though dance is associated with $\mathrm{HV}$, the pointe shoe, and the ballet technique is only a risk factor for $\mathrm{HV}$, not a conclusive cause. In more recent studies, features that were once believed to be associated with HV in dancers, are not. These include hours of dancing and hours of dancing en pointe per week, age at which pointe work was started and totally years on point.

\section{Akshai Mansingh \\ Affiliation: University of the West Indies}

\section{Posterior Ankle Impingement in Fast Bowlers}

Introduction: Ankle injuries in fast bowlers in cricket are common and can be acute or chronic in nature. Posterior ankle impingement is a collection of chronic conditions caused by repetitive forced dorsiflexion as well as plantar flexion. It is being reported increasingly in fast bowlers and is often associated with a large os trigonum.

This study of posterior ankle impingement in West Indian fast bowlers looks at clinical features in presentation and findings, and proposes treatment options. Factors influencing treatment will be discussed

Methods: A retrospective analysis of ankle impingement injuries treated in fast bowlers in the West Indies was conducted between 2005 and 2014. Duration and features of presentation were noted as well as investigation findings. Treatment effected was reviewed as well as the outcome.

Results: Seven fast bowlers had evidence of os trigonum in the front foot only. Pain was felt on forced plantar flexion and dorsiflexion on front foot landing; no pain was felt with running. Four had large os trigonum on radiographs, and one was detected only by MRI. Three bowlers with low workloads resolved with steroid injections. The remainder had surgical excision which led to recovery.

Conclusion: This injury is being seen increasingly in fast bowlers. Steroid injections are useful in bowlers with low workloads, but surgical excision is recommended in bowlers with heavy workloads. Further investigation is required in biomechanics of bowling to determine the cause for the increase in this condition.

RobRoy Martin

Affiliation: Duquesne University

Additional Authors: RobRoy Martin, Hal Martin, Ricardo SchrÃ đder

\section{Management of Deep Gluteal Syndrome -A case series}

Introduction: While deep gluteal syndrome (DGS) is becoming increasingly recognized, there is little information to guide the management of patients diagnosed with DGS. A positive outcome of those with DGS may require a comprehensive multi-professional treatment approach. The current study describes an algorithm for management of individuals with DGS and includes physical therapy, psychological counseling, intramuscular Computed Tomography (CT)-guided injection, and endoscopic surgery.

Material \& Methods:The cases series was conducted between April 2014 and December 2015. Study population was composed of subjects diagnosed with deep gluteal pain through a detailed history, physical examination, imaging and diagnostic testing. Six female subjects were treated during an average time of 20 weeks (6-36 weeks). Visual analog scale, modified Harris Hip Score, and patient satisfaction were used to describe outcomes. Physical examination was used to determine appropriate physical therapy interventions. Specifically, interventions were directed at lumbopelvic, hip intra-articular, hip extra-articular, and/or pelvic floor structures with neuromuscular retraining, soft tissue length-tension rebalancing, joint mobilization, soft tissue mobilization, and/or neural mobilization as indicated. Neuropsychiatry evaluation was used to determine appropriate psychological counseling techniques to address issues associated with anxiety and depression. Intra-muscular injections through CT guidance in the piriformis and/or obturator muscles were used in cases that did not have satisfactory results after physical therapy and psychological counseling. Subjects who found temporary benefit from injections were considered candidates for endoscopic surgery.

Results: The deep gluteal pain was identified as a chronic condition associated with factors such as low back pain, presence of hip intra-articular pathology, previous pelvic surgery, urogynecological and psychological influences. When appropriately managed improvement was obtained in the subjects included in this study within an average time of 20 weeks (6-36 weeks).

Discussion \& Conclusion: Patients with DGS can have a good outcome when the algorithm for appropriate management is applied. Thorough diagnostic work-up was found to be the best method to determine the most effective treatment. Conservative management should consist of a multiprofessional treatment approach with neuromuscular retraining, soft tissue length-tension rebalancing, joint mobilization, soft tissue mobilization, neural mobilization, psychological counseling, and/or intra-muscular injections as indicated through the comprehensive evaluation. 


\section{Effect of a long-term physical activity intervention on risk factors for coronary heart disease in adults with intellectual disability}

Background: Physical inactivity is a major risk factor for developing coronary heart disease (CHD) in persons with an intellectual disability (ID). Longevity of individuals with an ID can be ameliorated by decreasing the risk for CHD with increased physical activity. Limited research is available on long-term physical activity intervention in persons with ID therefore; the aim of this study is to determine the effect of a long-term physical activity intervention programme on risk factors for CHD.

Methods: A cohort of 74 participants living in a care facility in Potchefstroom, North West Province of South Africa, gave consent for participation in this seven-year follow-up study. The participants aged chronologically between 25 and 76 years were intellectually aged between 4 and 12 years. The CHD risk factors were determined by means of questionnaire and physical assessment of resting blood pressure, body mass index (BMI), fat percentage and non-fasting glucose and cholesterol measurements. The long-term physical activity intervention included two weekly supervised aerobic and resistance training activities for health improvement. ANCOVA with adjustment for baseline were performed as well as the McNemar exact test for pre- to post-intervention changes in risk factor variables and point prevalence of risk factors.

Results: The point prevalence of inactivity in the participation decreased from $50 \%$ to $24 \%$. Total cholesterol prevalence risk factor increased from $23 \%$ to $45 \%$. Prevalence of age as a risk for developing CHD increased significantly in the seven-year follow-up study from a $10 \%$ prevalence to $28 \%$. Body mass decreased significantly in men $(1.25 \pm 5.43 \mathrm{~kg})$ and increased significantly in women $(0.15 \pm 6.83 \mathrm{~kg})$. BMl reflected the changes in body mass for men and women respectively. Body fat percentages in men (2.98\%) and in women (0.95\%) increased. A significant increase in systolic blood pressure (SBP) for men $(6.2 \pm 18.1 \mathrm{mmHg})$ and diastolic blood pressure (DBP) for women $(6.35 \pm 10.42 \mathrm{mmHg}) \mathrm{was}$ determined. In women total cholesterol increased significantly $(0.53 \pm 0.41 \mathrm{mmol} / \mathrm{L})$.

Conclusion: A long-term physical activity intervention in a population with ID reduced the point prevalence of inactivity, while total cholesterol increased.

Arline Muller

Affiliation: Unizulu

Additional Authors: Arline Muller, Henriette Hammill

\section{The Effect of Pilates And Progressive Muscle Relaxation Therapy (Mrt) on Stress and Anxiety During Pregnancy: a Literature Review}

Aim: To compile a structured literature review pertaining to the effect of pilates and progressive muscle relaxation therapy (MRT) on stress and anxiety during pregnancy.

Methods: A literature search from 2005-2015 was conducted in order to capture all the articles applicable to the effects of pilates and progressive MRT on stress and anxiety during pregnancy. A descriptive analysis was undertaken of those articles that met the established criteria for inclusion following the search.

Results: Searches from the databases identified 4475 articles, of these, 4461 articles were excluded and 14 articles were ultimately included for review.

Conclusion: Additional research into this topic is necessary due to the limited number of papers that are published especially concerning the psychological and physiological effects of pilates during pregnancy. The literature on progressive MRT during pregnancy is better documented. The findings of this systematic review indicate that there is strong evidence that progressive MRT is an effective method in reducing stress and anxiety during pregnancy.

Key words: pregnancy, pilates, progressive muscle relaxation therapy, stress, anxiety.

Stacy Nellemann

Affiliation: Rhodes University

Additional Authors: Stacy Nellemann, Candice Christie, Jonathan Davy

\section{A laboratory simulated investigation into the impact of sunglass tint on the catching performance of fielders during cricket}

Introduction: Vision is particularly important for the successful execution of goal directed actions in sport. In order to accomplish this, spatial and temporal information provided by the eyes to the brain, results in the perception of the surrounding environment. In sport played outdoors, vision is further impacted by exposure to harmful UV radiation from the sun affecting ocular health, ultimately affecting the quality of vision. Consequently outdoor athletes like cricketers, who are the focus of this study, use sunglasses to prevent this from occurring. In addition, the tint of sunglasses purportedly provides performance-enhancing benefits such as contrast enhancement based on the colour of the lens. Despite these purported benefits, no research has established the efficacy of different colour tints of lenses. The primary objective of this study therefore is to investigate how catching performance of cricketers, is affected by different colour tints of sunglasses

Methods: In order to investigate the effect four different colour tints of sunglass (clear, red, grey and blue) affect catching when fielding in cricket, a within subjects, repeated measures design was used. Players were pre-screened for eligibility and required to be cricket players between the ages of 18-30yrs with a minimum of 5 years playing experience, no ocular deficiencies and be non-habitual sunglass wearers. During the pre-screening examination, objective ocular measures of contrast sensitivity, stereopsis and visual acuity were taken. Eligible players partook in four experimental sessions, which were randomised. Prior to each session, players were habituated wearing the selected tinted lens by catching 12 balls projected with a bowling machine. Following habituation players were required to catch 30 balls projected at a speed of $26 \mathrm{~m} . \mathrm{s}-1(93 \mathrm{~km}$.h-1) with an inter-delivery time of 32s. The quality of each catch was retrospectively graded using Wickstrom's Catching Performance Scale and the absolute number of catches made recorded. In the last experimental session, participants completed a questionnaire to investigate subjective opinion of performance comparing the different lenses. The findings will be clinically relevant not only to opticians but also to those interested in enhancing performance Results: Data is currently being collected therefore will be analysed and presented at the conference. 
Natalia Neophytou

Affiliation: Centre for Exercise Science and Sports Medicine, School of Therapeutic Sciences, Faculty of Health Sciences, University of the Witwatersrand, Johannesburg, South Africa

Additional Authors: Natalia Neophytou, Kerith D Aginsky, Kirsten Keen,

\section{The Comparison of change of Transversus Abdominis muscle thickness and plank holding time in Club Cricket Players}

Background: Research between bridge holding time as an indication of core stability, by means of measuring transverse abdominis (TA) muscle function is limited.

OBJECTIVE: Acorrelation study comparing TA muscle function and bridge holding time in club cricketers.

Methods: Seventeen male, premier league cricketers (age: $22.1 \pm 3.3$ years) participated. Visual ultrasound measured bilateral TA, internal oblique (OI) and external oblique (OE) muscle thickness at rest and during abdominal hollowing. Muscle function was measured as the change in muscle thickness from rest to abdominal hollowing and compared to holding time of the supine and prone bridges (seconds) by means of a Pearson's correlation.

Results: TA muscle thickness was preferentially recruited bilaterally $(p=0.0000)$. No significant correlations existed between TA muscle function and supine or prone bridge holding time. Negative correlations were found between prone bridge and ND TA muscle thickness at rest $(r=-0.57, p=0.017)$ and abdominal hollowing $(r=-0.54, p=0.03)$; and supine bridge with $D$ and ND OE at rest $(D: r=-0.52 ; p=0.04 ; N D: r=-0.60 ; p=0.01)$ and abdominal hollowing ( $D: r=-0.55, p=0.03$; ND: $r=-0.54, p=0.03$ ).

Conclusion: Prone and supine bridge holding times are not correlated with TA muscle function.

Natalia Neophytou

Affiliation: Centre for Exercise Science and Sports Medicine, School of Therapeutic Sciences, Faculty of Health Sciences, University of the Witwatersrand, Johannesburg, South Africa

Additional Authors: Natalia Neophytou, Estelle Watson, Justin Jeffrey, Amy Bedford, Craig Eliasov, Demitri Constantinou

\section{The use of Ekso BionicsTM Exoskeleton as a rehabilitation tool in patients with spinal cord injuries: Acase study series}

Background: Major advances in robotic technology have led to the development of various types of walking devices used for locomotor training.[3,4] One such device was famously used during the 2014 FIFA World Cup TM to kick the first ball of the tournament.[5] These various devices, although inspiring and encouraging for people living with paralysis, are very much in the early stages of development, and more evidence and practical information is required if clinicians are to safely use them as rehabilitative tools.

Objective: An exploratory study investigating the Exoskeleton as a rehabilitation tool in spinal cord injury patients.

Methods: Standardised baseline measurements were taken pre and post intervention for 3 spinal cord injury participants. The participants underwent 10 sessions of locomotive training using the Ekso ${ }^{\mathrm{TM}}$ Exoskeleton device over a 5 week period. An $80 \%$ compliance rate was required in order to complete the study. Pre and post measures included: calf circumference, resting blood pressure and the he Rand 36 item health survey. The survey was used to determine physical functioning(PF), role limitations due to physical health(RP), role limitations due to emotional problems $(\mathrm{RE})$, vitality/energy (VT), mental health/emotional well-being (MH), social functioning (SF), bodily pain(BP) and general health(GH) [10]. A composite score for the mental component and physical component scales were calculated.

Results: All of the cases showed much individuality in rates and progression of assisted walking. This intervention showed no clinically meaningful trends in heart rate, however, improved muscle bulk (increased calf circumference) was found in both participants. Improvement of between 8-10\% was found for mental quality of life; however physical outcome scores were conflicting. Indeed, the intervention showed a reduction in both physical functioning and increase in bodily pain in each case respectively.

Conclusion: Due to the current lack of knowledge of the physical demand of these types of devices, it is imperative that clinical guidelines, for progression of standing and walking time, are implemented.

Habib Noorbhai

Affiliation: Cape Peninsula University of Technology

Additional Authors: Habib Noorbhai, Timothy Noakes

\section{A qualitative evaluation of backlift batting techniques taught by cricket coaches}

Introduction: The art and science of cricket has continuously evolved in the last century. The straight backlift was institutionalised first in 1912 followed by the Marylebone Cricket Club's coaching manual in 1954. Until 2007, it was common knowledge to coach the straight backlift to non-elite cricketers. Presently, it is common practice for coaches to not consider the straight backlift as the only dogma. Our previous studies have shown that both successful elite batsmen and non-coached cricketers employ a lateral backlift and are able to score runs easily. Therefore, the aim of this study was to evaluate the teachings of the backlift batting techniques among multi-levelled cricket coaches.

Methodology: Multi-levelled cricket coaches $(n=120)$ from seven International Cricket Council (ICC) countries (South Africa, Australia, New Zealand, England, India, Pakistan and West Indies) were evaluated utilizing an online survey questionnaire through Google Forms. A mixture of 13 open and closed-ended questions were purposively asked to gauge instinctual insights of their coaching methods and perceptions. These questions were related to their demographics, insights of backlift batting techniques and implications of batting techniques. Both descriptive and inferential statistics were utilised to analyse categorical feedback with Statistica 11. The level of significance was set at $p<0.05$.

Results: A majority of cricket coaches $(70 \%)$ were not aware of what a looped or lateral backlift constituted of $(p<0.05)$. In addition, more than $70 \%$ of cricket coaches utilized either the straight backlift or the backlift in the directions of the slips in their coaching methods $(p<0.05)$. A distinctive finding was the variance of feedback received by all the cricket coaches. Based on this evaluation, it was found that cricket coaches are coaching the backlift differently. Some of the proposing quotes from the cricket coaches included: "one needs to teach the basics of batting to young cricketers by hitting the ball and treating each player as an individual". In contrast, opposing quotes from the cricket coaches included: "one needs to apply a generic approach when coaching batting technique to cricket players and it doesn't matter whether the face of the bat is open or closed".

Conclusion and Coaching Implications: This study has shown a distinct variance of coaching backlift batting techniques by $70 \%$ of multi-levelled cricket coaches. This is a slight concern, especially at grass-root level, as previous studies have shown that a straight backlift batting technique can limit the potential of a naturally gifted batsman. The way forward is to congregate all cricket coaches from the ICC coaching associations in order to educate and demonstrate why and how the lateral backlift batting technique is a strong determinant for a batsman's success, at all levels.

Keywords: Cricket, backlift batting techniques, cricket coaching, qualitative evaluation 
Habib Noorbhai

Affiliation: Cape Peninsula University of Technology

Additional Authors: Habib Noorbhai, Prof Leon van Niekerk

\section{A scientific and psychological analysis of the Proteas performances during the last seven Cricket World Cups}

The South African cricket team comprise of some of the most highly ranked players, yet despite this, one could say that they have had an unfavourable streak at the past seven Cricket World Cups having not reached a World Cup final. This paper aims to critique the assumptions behind the Proteas' performances during several World Cup campaigns and to show that the lay press original label of 'chokers' for the past two decades is not an accurate description. Terms such as 'Choking', the 'Yips' and other psychological terminology such as stereotype threat that apply to elite sportsmen need to be differentiated as these vary. In addition, an analysis of the Proteas performances over the years are performed and associated factors are discussed. It must be highlighted that we should be asking different questions instead of providing different answers to the same questions. Further research should be conducted at real-time game situations among the Proteas cricket team and other national teams, in order to provide an effective intervention approach. This would further enhance an understanding of the team dynamics prior to knockout tournaments such as the ICC Cricket World Cup.

\section{S.O. Onagbiye}

Affiliation: Physical Activity, Sport and Recreation Focus Area, North-West University, Potchefstroom

Additional Authors: S.O. Onagbiye, S.J. Moss, M. Cameron

Managing non-communicable diseases in an African community: Effects, compliance, and barriers to participation in a four-week exercise intervention.

Introduction: To determine the compliance, barriers, and effects of participation in a four-week exercise intervention to reduce risk factors for NCDs among Setswana-speaking community-dwelling Potchefstroom adults from a low resourced area of South Africa.

Methods: An exercise program and associated pre-post test were performed by 76 participants (men, $n=26$ and women, $n=50$ ) aged 35 to 65 years. Baseline and end tests included height, weight, hip and waist circumference, heart rate, blood pressure, glucose, cholesterol, quality of life, and cardiorespiratory fitness measurements. The intervention consisted of three days/week combined aerobic and resistance exercise at an intensity of $70 \% \mathrm{HRR}$ as determined at baseline. Compliance and barriers to participation were determined post-intervention by means of attendance registers and interviews. ANCOVA with adjustment for pre-test was performed for all repeated variables. The Cronbach's alpha coefficients for exercise benefits were 0.81 and for barriers 0.84

Results: Of the 26 men ( $40.8 \pm 5.45$ years) and 50 women ( $43.6 \pm 7.8$ years) recruited, 54 completed the intervention ( $71 \%$ compliance). The fourweek aerobic exercise intervention significantly reduced body mass, RPE and MCS in men, and body mass, BMI, VO2max, RPE, glucose, PCS and MCS in women. Participants reported that the exercise milieu as a major barrier to exercise compliance while the interviews reported lack of time.

Conclusion: A one month exercise intervention elucidated positive changes in risk factors for NCDs in a low-resource community. A drop-out rate of $29 \%$ in this study is consistent with other exercise intervention trials. Exploration of the reported barriers may be useful for planning to increase compliance with future programs.

Keywords: Physical activity intervention, cardiorespiratory fitness, quality of life, non-communicable diseases, adults, Setswana

Adedayo Osholowu

Affiliation: Elite Health Systems (EHS) Sports Medicine, Orthopaedic Surgery And Rehabilitation Clinic

Additional Authors: Adedayo Osholowu, Onimisi Sunday Salami, Jimisayo Osinaike

\section{Arthroscopic repair following a Menisci Tear in two Nigerian Footballers: A case Report.}

Introduction: Reports of Arthroscopic Menisectomy and other minimally invasive orthopedic repairs are rare in Nigeria and the rest of Sub Sahara Africa. Most athletes have to undergo open Arthrotomy or continue to be managed with conservative options greatly hindering return to full performance and sometimes leading to the loss of a career and sometimes a means of livelihood for a family. A database search of Medline, Cinhal, and Sports Discus did not reveal any such cases, as most of the literature from Sub Saharan Africa is focused on Musculoskeletal Trauma and Infections. This report documents the 2 cases of Arthroscopic Menisectomy in Young Nigerian Football players. It details the surgical repair and management and the physiotherapy rehabilitation to full function and return to play.

Presentation of Case: This case report presents two male footballers who presented to our clinic with complaints of pain on the lateral aspect of the right knee which was sufficient to prevent him from playing. In both cases Musculo-Skeletal Ultrasound of the knee showed a Tear of the posterior horn of the medial meniscus shown as hypoechoic lines with extension to the articular surfaces. Magnetic resonance imaging (MRI) showed signal unequivocally reaching the surface of the meniscus further confirming the diagnosis. Arthroscopic debridement and lateral partial menisectomy was applied to both knees under spinal anesthesia. In one case arthroscopy revealed a lateral collateral Ligament tear which was repaired with a allograft.

Discussion: While there a many cases of Meniscus damage in the sports community in Nigeria and the rest of Sub Saharan Africa, arthroscopic repairs or meniscectomies have rarely been reported in literature. Generally most cases are managed by conservative methods involving physiotherapy and pain management which in cases where this is not effective will result in a reduction in performance during sport and early onset arthritis. Football is considered a high-risk sport for knee ligament and meniscal injuries. In the developed world a significant number of these patients undergo anterior cruciate ligament (ACL) reconstruction or arthroscopic meniscal repair. In contrast, there has been little mention of the role of arthroscopy or the treatment of sports-related injuries within the scope of musculoskeletal care in Nigeria and the rest of Sub Saharan Africa. We report here our experience performing Arthroscopic surgery on 2 athletes in Nigerian. Our goal in this report is to begin the discussion in the orthopedic community regarding when it is appropriate to introduce arthroscopy to the sports community in Nigeria and how to best make it sustainable.

Conclusion: Arthroscopic surgery can be successfully applied in the treatment of Meniscal tears. The advantages of the method include good visualisation during surgery, low morbidity and early healing and return to sport. With recent advances in arthroscopic techniques and methods, the indications for arthroscopic treatment must be extended to the sports community in Nigeria and the rest of Sub Saharan Africa. 


\section{Accommodation and job assignment for impaired or disabled workers}

Some compensation systems do not encourage permanently restricted workers who have been disabled due to work-related injuries to return to work. However, appropriate job placement of impaired workers has been shown to result in feelings of independence, usefulness and responsibility, as well as financial security. Physically impaired employees in many workplaces have experienced job discrimination because colleagues and supervisors assume that their work performance will be affected by physical limitations resulting from their disability. This theoretical paper discusses the possibilities of assigning workers to appropriate jobs based on their specific capabilities and limitations. The option of providing certain job accommodations in the form of workplace restructuring is also discussed. An ergonomics approach to designing industrial workstations attempts to achieve a balance between worker capabilities and task requirements to optimize worker productivity, as well as provide physical and mental wellbeing, job satisfaction and safety. For impaired workers, additional considerations need to be given to their functional limitations and altered physical capabilities. In some cases, modifications or alterations may need to be made to existing workplaces to accommodate these capabilities.

This paper investigates the possibility of evaluating specific worker limitations and capabilities through a thorough functional analysis, and matching these to task demands determined through a thorough job analysis at the workplace, to achieve the best possible productivity and worker well-being.

Lervasen Pillay

Affiliation: University of Witwatersrand, CESSM

Additional Authors: Lervasen Pillay, Prof Demitri Constantinou, Prof Yoga Coopoo,

\section{Arthritic patients' views and perceptions on exercise as an adjunct treatment regime for managing their condition.}

Objectives: This study aimed to assess the views of arthritic patients towards exercise as an adjunct treatment modality in managing their condition, the incidence of different healthcare providers in prescribing exercise, the exercise types and frequencies and the outcome of the effects of exercise on pain and activities of daily living activity.

Methods: A cross sectional survey study design was used. Patients of private general practitioners and a biokineticists' based in the southern suburbs of Johannesburg were invited to participate in the survey to assist with the collection of data. Questions were developed to determine various aspects of the effect of exercise and patient attitudes toward exercise as an additional management tool in patients with arthritic disease falling within the inclusion criteria. Secondary to this, information on the type of healthcare providers prescribing exercise, exercise types and outcomes of exercise were also collected. Outcomes were measured based on the patient's subjective responses based on improvements of the participants' arthritic conditions symptoms and activities of daily living with exercise.

Results: A total of 67 patients were surveyed. Males counted for $25.4 \%$, females $73.1 \%$. Age distribution was as follows: $59.7 \%(\mathrm{~N}=40)>50$ years of age, $35.8 \%(\mathrm{~N}=24) 30-50$ years of age and the remaining $4.5 \%(\mathrm{~N}=3)<30$ years of age. Most respondents suffered from osteoarthritis $(\mathrm{N}=29)$, followed by rheumatoid arthritis $(\mathrm{N}=27)$, gout $(\mathrm{N}=5)$ and post traumatic arthritis $(\mathrm{N}=3)$ while the remainder were not specific. Exercise was prescribed by doctors (but not specific enough), followed by physiotherapists then biokineticists. Exercise alone improved pain and activities of daily living by $11.1 \%$, while medication improved $21.1 \%$ while vast improvement was noted in the combination of exercise and medication (32.5\%).

Conclusion: Observations and analysis from the study concludes that there is benefit of exercise in treating arthritic disease best in combination with medical treatment. Patients believe that exercise assists them in managing symptoms of arthritis. Therefore it is an important adjunct treatment modality. Doctors were in fact advising exercise more than other healthcare providers but this advice was very non-specific. Limitation of this study was the small number of participants. Future research should be directed toward exploring the reasons why healthcare practitioners are not specific in their exercise advice. This study is consistent with other studies in this field of study.

Keywords: management of arthritic disease, exercise prescription, exercise as an adjunct treatment modality, exercise and pain relief, exercise and arthritis

Lee Pote

Affiliation: Rhodes University

Additional Authors: Lee Pote, Candice Christie

\section{Strength and conditioning practices of university and high school level cricket players: a South African context}

Introduction: Cricket is one of the world's major team sports, however it has received very little research attention, particularly with regards to the physical demands of the game. Furthermore, no studies have critically examined the strength and conditioning practices of cricket players. Therefore, the purpose of this study was to examine the strength and conditioning practices of school boy and University level cricket players in South Africa. This is important so that current practices that are being implemented can be adapted or changed accordingly to reduce the risk of injury and improve player performance.

Methods: The participants recruited for this study were the head strength and conditioning coaches or coaches (in the case of no strength and conditioning specialist), at 12 university and 50 high school teams. The university teams selected were based on their participation in the Varsity Cup Cricket and University Sport of South Africa (USSA) competitions. The high school teams chosen were the top 50 in the country at the time (rankings as of 9th March 2015). Participants were required to complete an online survey that was based on previous questionnaires for strength and conditioning research as well as cricket specific and injury statistics questions. The survey itself consisted of three main sections:

General questions which included background information on the coaches as well as their degrees, diplomas and qualifications. This section also focused on general conditioning practices such as physical testing, flexibility, speed and agility development as well as plyometric and resistance training.

Cricket specific questions focusing on conditioning practices and player workload monitoring for batsmen, bowlers, fielders and wicket keepers.

Injury and injury prevention questions, including injury monitoring, prevention exercises and common injury sites for batsmen, bowlers, fielders and wicket keepers

Results: Data are still being collated 


\section{Prevalence of Dietary Supplement Intake and Influencing Factors among Gymnasium Attendees in Pretoria}

Introduction: Studies report high prevalence of dietary supplement intake amongst gymnasium attendees worldwide. To date, no research has been conducted on this issue in a South African city. Objectives: To determine the prevalence of dietary supplement intake in individuals who exercise regularly in gymnasiums in the city of Pretoria, South Africa, and discuss the influencing factors. Furthermore, to compare results with those obtained in a study conducted in Belo Horisonte, Brazil, utilising the same questionnaire.

Methods: The questionnaire was obtained and translated from Portuguese into English. Individuals ( $n=269$, aged 31.5 \pm 10.7 y; men = 150; women $=119$ ) frequenting 17 gymnasiums throughout Pretoria were recruited. After obtaining informed consent, participants completed the questionnaire. Descriptive statistics and chi-square tests were performed.

Results: Exercise participation comprised mostly aerobic (76.6\%) followed by anaerobic exercise (62.1\%). Reasons for exercising included healthy habit $(70.6 \%)$, to lose weight $(42.8 \%)$ and to gain muscle mass (51.3\%). Dietary supplement use was high $(71.4 \%)$. The highest intake was in men $(60.9 \%)$. The two products with the highest consumption rate were vitamins and minerals $(43.1 \%)$ and supplements rich in protein (35.3\%). Women ingested more meal replacement shakes and natural herbal products than men. The majority of men took supplements in order to gain muscle mass and improve performance, whereas the majority of women took supplements for weight loss / fat burning, to replace meals and prevent future illness. More than half $(51.6 \%)$ of the participants used dietary supplements through their own initiative. Very few of them consulted a doctor (4.2\%) or dietician $(5.2 \%)$. The intake of dietary supplements was more common in participants $<30$ years $(54.2 \%)$. Conclusion: The study shows a high prevalence of dietary supplement intake amongst individuals frequenting gymnasiums in Pretoria. The majority of the dietary supplement intake is self-regulated. Results are comparable to the Brazil study.

Marlene Schoeman

Affiliation: University of the Free State

Additional Authors: Marlene Schoeman, Rudi de Wet, Louis Holtzhausen

Clinical, Haematological and Biochemical Characteristics of South African Gold-Miners Presenting with Exercise-Associated Muscle Cramps, Part 1: Background and Methodology

Introduction: Consensus on the pathogenesis of exercise associated muscle cramps (EAMC) has not been reached. Two main schools of thought exist, either proposing that (1) dehydration and electrolyte depletion or (2) altered neuromuscular control with muscle fatigue cause EAMC. Several factors within these hypotheses are conflicting and unexplained, possibly because of a lack of good data, making it difficult to inform evidenced based practices for the prevention of EAMC. Underground miners are especially prone to muscle cramps, yet no data were available to gain insight into the pathogenesis of EAMC in this population group. The aims of this study were to describe environmental, biochemical and haematological variables in gold miners with EAMC and compare it with a control group of miners without EAMC. The study consisted of two parts (retrospective and prospective) which required detailed methodological considerations and yielded extensive results and conclusions. Therefore, this study will be presented in two parts. Part 1 will focus on the background and methodology, while Part 2 will focus on the results and conclusions.

Methodology: The retrospective descriptive study involved underground mine workers who presented with EAMC over 18 months in a SA gold mine (CRA group). The prospective study consisted of a collection of biological data and blood profiles before (CONPRE) and after (CONPOST) 8 hour shifts on a volunteer group of underground mine workers not presenting with EAMC to generate normative data for underground mine workers and comparative data for the CRA group. Participants were classified into four groups based on the functional nature of the specific job descriptions comprising physical stresses such as vibration, cramped body positions for prolonged periods, high physical exertion and distance walking. Despite the large number of CRA participants, data were generally non-parametric.

Results: Results and discussions are categorised under hydration, electrolyte disturbances, muscle damage and inflammation to align with aspects from the two schools of thought regarding EAMC.

\section{Marlene Schoeman}

Affiliation: Sport and Exercise Medicine, University of the Free State

Additional Authors: Marlene Schoeman, Ceri Diss, Siobhan Strike

\section{Unilateral jump mechanics in transtibial amputees}

Introduction: Jumping is a fundamental skill in recreational sport and offers great potential for stimulating osteogenesis in osteoporosis prone amputees. While a reasonable body of research on amputee walking and running gait has accumulated to identify typical compensations and adaptations for amputees, a dearth of literature on amputee jumping exists. Jumping is mechanically different and more demanding than walking and running with a greater vertical force component. The aim of this study was to address the lack of knowledge on amputee jumping by exploring the underlying mechanics of performing the unilateral countermovement jump.

Methods: Six recreationally active unilateral transtibial amputees (TTAs) took part. Amputees were included if they were at least 1 year postamputation with no secondary pathology and had an amputation of a traumatic nature. All TTAs wore patellar tendon-bearing sockets with rigid pylons and their own prescribed prosthetic feet. For comparison, ten healthy able bodied $(A B)$ persons of the same age range and activity levels participated in the study. All participants wore their own athletic footwear. Participants performed ten maximal unilateral countermovement jumps with arms akimbo and approximately 30 seconds rest between each trial. The single jump with the greatest vertical position of the centre of mass was used for analysis. Data were collected using two piezoelectric force platforms synchronized with a 9-camera Vicon motion analysis system. AMannWhitney $U$ test was used to test for statistically significant differences $(p<0.05)$ between the TTA and AB participants.

Results: Very little strain energy $(-0.11 \pm 0.04 \mathrm{~J} . \mathrm{kg}-1)$ was stored in the prosthetic keel compared to the $A B$ intact ankles $(-0.28 \pm 0.09 \mathrm{~J} . \mathrm{kg}-1)$ during the countermovement to be returned as positive work during propulsion and effectively contribute to the jump. Generally, TTAs displayed similar knee and hip RoMs to the AB participants. Significantly reduced $(p=0.000)$ ankle RoMs were accompanied by significantly reduced $(p=0.016)$ peak ankle "plantarflexor" moments $(1.99 \pm 0.41 \mathrm{Nm} . \mathrm{kg}-1)$, represented by the passive recoil of the prosthetic keel. Average knee extensor moments $(1.35 \pm 0.61 \mathrm{Nm} . \mathrm{kg}-1)$ were significantly reduced $(\mathrm{p}=0.031)$ and the average hip extensor moments $(2.48 \pm 1.04 \mathrm{Nm} . \mathrm{kg}-1)$ as well as positive $\mathrm{knee}$ $(0.59 \pm 0.32 \mathrm{~J} . \mathrm{kg}-1)$ and hip work $(0.88 \pm 0.53 \mathrm{~J} . \mathrm{kg}-1)$ were mostly similar to those of the AB participants.

Conclusions: Despite being exposed to a greater vertical load compared to gait, insufficient strain energy was stored in the prostheses during the countermovement, brought about by the lack in RoM and compression of the cantilevered spring of the prosthetic shank. In contrast to walking and running gait, no clear compensations were noted for the reduced prosthetic RoM and peak plantarflexor moments at the knee and hip, highlighting the fact that compensations seen for TTAs in gait cannot be extrapolated to jumping to inform this aspect of recreational sport, prehabilitation and rehabilitation for amputees. 
Nicola Sewry

Affiliation: University of Cape Town, Division of Exercise Science and Sports Medicine

Additional Authors: Nicola Sewry, Sharief Hendricks, Mike Lambert, Bevan Matthews, Brad Roode

\section{The Velocity of Ball-Carriers and Tacklers during Shoulder Tackles}

The most frequently executed type of tackle in rugby is the shoulder tackle. A shoulder tackle occurs when the tackler uses either shoulder as the first point of contact with the ball carrier. The aim of this study was to compare the velocity of the ball-carrier and tackler engaged in shoulder tackles, and determine whether factors such as the number of passes from the previous phase, match period, quality of attack, match location and position of tackler had an effect on the mean and approaching velocities of the ball-carrier and tackler. The velocity of of the ball-carrier and tackler during shoulder tackles $(n=12)$ were determined using a $2 \mathrm{D}$ analysis tool. The tackles were also coded according to pass number, match period, quality of defence and attack, match status, defensive shape and movement, position of tackler and ball-carrier. The ball-carrier's velocity (4.10 $\pm 1.85 \mathrm{~m} . \mathrm{s}-1)$ when entering contact was significantly higher than the tackler's velocity $(5.19 \pm 3.22 \mathrm{~m} . \mathrm{s}-1)$. The ball-carrier's velocity was significantly higher when entering contact further from the set piece $(p<0.001)$, when playing away from home $(p<0.001)$ and when ranked in the top three $(p<0.001)$. The findings of this study provide insight into the complexities of the tackle in rugby.

\section{Takshita Sookan}

Affiliation: University of KwaZulu Natal

Additional Authors: Takshita Sookan, Andrew McKune, Michael Ormsbee, Jose Antonio, Nombulelo Magula, Umesh Lalloo, Ayesha Motala

\section{Effect of a progressive resistance training program and whey protein intake on quality of life in human immunodeficiency virus infected} individual receiving antiretroviral therapy.

Introduction: Advances in HIV treatment in the last three decades has resulted in improved health, prolonged life and substantially reduced the risk of HIV transmission. [u1] This impact broadens to all facets of life, influencing quality of life (QOL). Progressive resistance training (PRT) combined with effective supplementation can increase muscle mass and improve physical performance in persons with HIV infection and may enhance outcomes in these patients as well as improve QOL.

Methods: Forty HIV infected participants $(40.8 \pm 7.7 \mathrm{yrs}, 70.8 \pm 16 \mathrm{kgs}$, BMI $30.9 \pm 7.2 \mathrm{~kg} . \mathrm{m} 2)$ receiving ART ( $\geq 18$ months) were randomly assigned to either a whey protein/resistance training (RT) group $(n=18)$, placebo/PRT group $(n=14)$ or control group $(n=8)$. Participants received either $20 \mathrm{~g}$ whey or placebo (maltodextrin) pre and immediately post each RT workout. Whole body RT was performed $2 /$ week for 12 weeks. To assess QOL the WHOQOL-HIV BREF 31 was used which has six domains: physical, psychological, level of independence, social relationships, [u2] environment, and spiritual. The questionnaire was completed at baseline and then at 12 weeks. The mean score of questions within each domain was used to calculate the domain score. Statistical analysis consisted of a two-way ANOVA and Sidak's multiple comparison post hoc testing. Alpha was set at $P \leq 0.05$.

Results: The Physical Domain showed a significant time effect (TE) $(P=0.02)$ with the placebo group increasing from baseline (17.69a.u.) to post (19.15a.u) (Mean difference -1.5a.u., 95\% Cl -2.9 to -0.05a.u.). There were no significant changes in the supplement group. The Social Relationships Domain exhibited a significant TE $(P=0.02)$ with the placebo group increasing significantly from 14.23a.u. to 16.54a.u post (Mean difference -2.3a.u., 95\% Cl -3.9 to -0.7a.u.). Environment Domain showed a significant TE $(\mathrm{P}=0.002)$ with both placebo (15.65a.u. to 16.46a.u.; mean difference -0.8 a.u., $95 \% \mathrm{Cl}-1.5$ to -0.09 a.u.) and supplement groups (14.35a.u. to $15.03 a . u$. .; mean difference -0.7 a.u., $95 \% \mathrm{Cl}-1.3$ to 0.04 a.u.) demonstrating significant increases from baseline after 12 weeks of training. The Spiritual Domain indicated significant $T E(P=0.05)$ with the placebo group increasing from baseline (16.38a.u.) to post (17.92a.u) (Mean difference $-1.5 a . u$., $95 \% \mathrm{Cl}-2.8$ to $-0.3 a . u$.). There were no significant changes in the supplement group. There were no changes in the Psychological or Level of Independence Domains for the placebo and supplement groups. There were no significant changes in the control group for any of the six domains.

Conclusion: Several components of QOL improved in ART treated HIV infected individuals that participated in the PRT program. Changes were predominately shown in the placebo group (Domains $1,4,5,6$ ). This can be attributed to positive social and environmental effects of exercise programs. Exercise training is an inexpensive and efficacious strategy for improving QOL in this population with can impact other facets of their lives.

\section{Sharhidd Taliep}

Affiliation: Department of Sport Management, Faculty of Business and Management Sciences, Cape Peninsula

Additional Authors: Sharhidd Taliep, Ruan Rust, Njabulo Mhlongo, Janine Gray, Catherine Draper, Sherylle Calder, Christi Botha

\section{The relationship between ethnicity, socio-economic status and visual skill in adolescent cricket batsmen}

Introduction: This study aims at identifying a reason for the poor batting performance of Black African players by investigating the relationship between ethnicity, socio-economic status and visual skills.

Methods: Fifty-one adolescent male skilled cricket batsmen consisting of three different racial groups were recruited. The participants completed the 3 phases of visual skill tests. Phase 1: Simple visual motor skills were assessed using the Beery-Buktenica Test. Phase 2: Visual skills important for ball sport performance were assessed using saccadic eye movement, visual tracking, depth perception, eye-hand reaction/eye-hand coordination tests. Phase 3: Visual skills important for cricket batting performance were assessed using a temporal occlusion technique to test visual perceptual and anticipatory decision making. In the temporal occlusion task, batsmen had to predict the swing and length of the delivery at various occlusion times. Socio-economic status of players was recorded using a 17 -point asset score.

Results: Phase 1: Black African batsmen scored significantly worse than Coloured/Indian and White batsmen in the Beery-Buktenica Test. Phase 2: Black African batsmen performed significantly worse that Coloured/Indian batsmen in the saccadic eye movement, eye tracking, and eye-hand reaction/eye hand coordination test and significantly worse than White batsmen during the eye tracking test. Phase 3: Black African batsmen were significantly worse than White and Coloured Indian batsmen at predicting swing at 3 occlusion times (200ms after ball release, $300 \mathrm{~ms}$ after ball release and at ball bounce). For the prediction of length, BlackAfrican batsmen were worse than White and Coloured/Indian batsmen at $200 \mathrm{~ms}$ after ball release. There were no significant differences between the Coloured/Indian and White batsmen for all phases of visual skills testing. There was a significant positive correlation between socio-economic status and all three phases of tests.

Conclusion: BlackAfrican batsmen had poorer visual skills than White and Coloured batsmen for all phases of visual skill tests providing a possible reason for their poor batting performance. Poor visual skills is associated with low socio-economic status as these players seldom have access to sporting schools, facilities, structured training programmes and coaches, which could hinder their development as batsmen. We therefore emphasise the importance of including visual skills training to all coaches, trainers and exercise specialist working with adolescent cricket players. 


\title{
Release parameters of the basketball jump shot across shooting ability in Zimbabwe male league basketball players
}

Introduction: Basketball is a very popular game in Zimbabwe and all over the world. Shooting, a key skill in basketball, is mostly done as a jump shot (JS). The low conversion rate of the JS in league basketball matches suggests that Zimbabwean league basketball players have problems in executing the JS. This study described successful JS in terms of release parameters (RPs) (height, velocity, angle and spin) across levels of shooting ability (good, average and poor) and further investigated if significant differences existed in the RPs across levels of JS shooting ability.

Methods: Twenty six players, purposively sampled from the Bulawayo Basketball Association, made ten successive JSs each from the free throw line, after a warm up. These shots were videotaped, using standard videography protocols. A Panasonic VDR D1160 camera placed 10.7 metres away from, and perpendicular to the plane of motion of the subjects, mounted on a tripod 1.9 metres high, was used to videotape the JS. The JS videos were cut using ANYCONVETER software. Quintic Coaching $4.02 \mathrm{v} 17$ software was then used to compute RPs for each of the cut videos.

Results: The study indicated that players' successful JS for good, average and poor players were executed at release angles of $49.5 \pm 7.6^{\circ}, 56.9 \pm 9.0^{\circ}$, $55.4 \pm 7.6^{\circ}$; at release velocities of $5.0 \pm 1.3 \mathrm{~m} / \mathrm{s}, 5.7 \pm 1.7 \mathrm{~m} / \mathrm{s}, 5.6 \pm 1.7 \mathrm{~m} / \mathrm{s} ;$ from absolute release heights of $2.5 \pm 0.3 \mathrm{~m}, 2.6 \pm 1.1 \mathrm{~m}, 2.4 \pm 0.3 \mathrm{~m} ;$ with relative release heights of $1.4 \pm 0.1 \mathrm{~m}, 1.4 \pm 0.1 \mathrm{~m}, 1.4 \pm 0.2 \mathrm{~m}$ and at release spins of $3.6 \pm 1.6 \mathrm{~Hz}, 3.3 \pm 1.5 \mathrm{~Hz}, 3.2 \pm 1.4 \mathrm{~Hz}$, respectively. The study also found statistically significant differences $(p \leq 0.05)$ in the release angle, and vertical component of velocity across JS ability.

Conclusion: It was concluded that coaches in the Bulawayo Basketball league need to design practises that develop optimal values of release angle and vertical component of release velocity when training for JS.

Key Words: basketball, jump shot, release parameters

\section{Sharhidd Taliep}

Affiliation: Department of Sport Management, Faculty of Business and Management Sciences, Cape Peninsula

Additional Authors: Sharhidd Taliep, Ruan Rust, Njabulo Mhlongo, Janine Gray, Catherine Draper, Sherylle Calder, Christi Botha

\section{The relationship between ethnicity, socio-economic status and visual skill in adolescent cricket batsmen}

Introduction: This study aims at identifying a reason for the poor batting performance of Black African players by investigating the relationship between ethnicity, socio-economic status and visual skills.

Methods: Fifty-one adolescent male skilled cricket batsmen consisting of three different racial groups were recruited. The participants completed the 3 phases of visual skill tests. Phase 1: Simple visual motor skills were assessed using the Beery-Buktenica Test. Phase 2: Visual skills important for ball sport performance were assessed using saccadic eye movement, visual tracking, depth perception, eye-hand reaction/eye-hand coordination tests. Phase 3: Visual skills important for cricket batting performance were assessed using a temporal occlusion technique to test visual perceptual and anticipatory decision making. In the temporal occlusion task, batsmen had to predict the swing and length of the delivery at various occlusion times. Socio-economic status of players was recorded using a 17-point asset score.

Results: Phase 1: Black African batsmen scored significantly worse than Coloured/Indian and White batsmen in the Beery-Buktenica Test. Phase 2: Black African batsmen performed significantly worse that Coloured/Indian batsmen in the saccadic eye movement, eye tracking, and eye-hand reaction/eye hand coordination test and significantly worse than White batsmen during the eye tracking test. Phase 3: Black African batsmen were significantly worse than White and Coloured Indian batsmen at predicting swing at 3 occlusion times (200ms after ball release, 300ms after ball release and at ball bounce). For the prediction of length, Black African batsmen were worse than White and Coloured/Indian batsmen at 200ms after ball release. There were no significant differences between the Coloured/Indian and White batsmen for all phases of visual skills testing. There was a significant positive correlation between socio-economic status and all three phases of tests.

Conclusion: Black African batsmen had poorer visual skills than White and Coloured batsmen for all phases of visual skill tests providing a possible reason for their poor batting performance. Poor visual skills is associated with low socio-economic status as these players seldom have access to sporting schools, facilities, structured training programmes and coaches, which could hinder their development as batsmen. We therefore emphasise the importance of including visual skills training to all coaches, trainers and exercise specialist working with adolescent cricket players.

\author{
Eberhard Tapera \\ Affiliation: National University of Science \& Technology \\ Additional Authors: Eberhard Tapera, Lateef O.Amusa
}

\section{Release parameters of the basketball jump shot across shooting ability in Zimbabwe male league basketball players}

Introduction: Basketball is a very popular game in Zimbabwe and all over the world. Shooting, a key skill in basketball, is mostly done as a jump shot (JS). The low conversion rate of the JS in league basketball matches suggests that Zimbabwean league basketball players have problems in executing the JS. This study described successful JS in terms of release parameters (RPs) (height, velocity, angle and spin) across levels of shooting ability (good, average and poor) and further investigated if significant differences existed in the RPs across levels of JS shooting ability.

Methods: Twenty six players, purposively sampled from the Bulawayo Basketball Association, made ten successive JSs each from the free throw line, after a warm up. These shots were videotaped, using standard videography protocols. A Panasonic VDR D1160 camera placed 10.7 metres away from, and perpendicular to the plane of motion of the subjects, mounted on a tripod 1.9 metres high, was used to videotape the JS.The JS videos were cut using ANYCONVETER software. Quintic Coaching 4.02 v17 software was then used to compute RPs for each of the cut videos.

Results: The study indicated that players' successful JS for good, average and poor players were executed at release angles of $49.5 \pm 7.6^{\circ}, 56.9 \pm 9.0^{\circ}$, $55.4 \pm 7.6^{\circ}$; at release velocities of $5.0 \pm 1.3 \mathrm{~m} / \mathrm{s}, 5.7 \pm 1.7 \mathrm{~m} / \mathrm{s}, 5.6 \pm 1.7 \mathrm{~m} / \mathrm{s}$; from absolute release heights of $2.5 \pm 0.3 \mathrm{~m}, 2.6 \pm 1.1 \mathrm{~m}, 2.4 \pm 0.3 \mathrm{~m} ;$ with relative release heights of $1.4 \pm 0.1 \mathrm{~m}, 1.4 \pm 0.1 \mathrm{~m}, 1.4 \pm 0.2 \mathrm{~m}$ and at release spins of $3.6 \pm 1.6 \mathrm{~Hz}, 3.3 \pm 1.5 \mathrm{~Hz}, 3.2 \pm 1.4 \mathrm{~Hz}$, respectively. The study also found statistically significant differences $(p \leq 0.05)$ in the release angle, and vertical component of velocity across JS ability.

Conclusion: It was concluded that coaches in the Bulawayo Basketball league need to design practises that develop optimal values of release angle and vertical component of release velocity when training for JS.

Key Words: basketball, jump shot, release parameters 
Jason Tee

Affiliation: University of Johannesburg

Additional Authors: Jason Tee, Yoga Coopoo, Mike Lambert

\section{GPS comparison of training activities and game demands of professional rugby union}

Introduction: Closely matching training session exertions with actual match play intensities ensures players are physically prepared for competition, and may prevent overtraining.

Methods: The movement patterns of four typical rugby union training activities (traditional aerobic, high intensity interval, game based and skills training) were compared with match play using global positioning systems (GPS). The degree of difference from match play was determined by calculating Cohen's effect size statistic. Training activities for players in different positions were similarly assessed. Movement patterns were measured as relative distance, distance walking (0-2m.s-1), jogging (2-4m.s-1), striding (4-6m.s-1) and sprinting (>6m.s-1), and sprint and acceleration (>2.75m.s-2) frequency.

Results: Game based training is the training activity most similar to match play, but doesn't meet all match requirements for all positions. For example, there are large differences in relative distance, striding distance, sprint distance and acceleration frequency between game based training and match play for scrumhalves.

Conclusion: If game based training is used as the primary training activity, position specific supplementary training is required to ensure players are adequately prepared for the demands of match play.

\section{Georgia Torres}

Affiliation: SASMA

\section{Effect of Exercise Intervention Programs on persons with and without Metabolic Syndrome}

Background: Effective exercise programs for treating metabolic syndrome (MetS) have not been devised. The principle aim of this study was therefore to use the anaerobic threshold (AT) to design an exercise program that optimized individual exercise responses in individuals with MetS.

Methods: Ten participants with MetS (MetSL) exercised using a walking program which does not use the AT to set training intensities. A second group of ten participants without MetS exercised using velocity at AT to set training intensities (Non-MetSV). The experimental group consisted of ten participants with MetS exercising using velocity at AT to set training intensities (MetSV). Physical, physiological and metabolic responses were measured in all groups before, during and after twenty weeks of exercise.

Results: BMI and waist circumference decreased whilst velocity at AT increased in all training groups. The VO2 peak did not change significantly in the non-MetSV group. The blood pressure response was favourable in the groups with MetS yet absent in the group without MetS. The MetSV group was the only group to show significant, positive changes in any of the metabolic parameters (fasting insulin and HOMA). In addition, the training program used in the MetSV group had a greater effect on reducing the number of MetS components than did the training program not using AT.

Conclusion: An exercise program using AT to set intensity is effective in eliciting favourable responses in individuals diagnosed with MetS while allowing a bearable exercise intensity and duration in subjects unused to the discipline of exercise training.

Monique Tredoux

Affiliation: DSM/ATLife (Dietician)

\section{Oat beta-glucan: An alternate cholesterol management nutrient for athletes}

The results from the SANHANES (South African National Health and Nutrition Examination Survey) data published in August 2013 typically display a cholesterol crisis. One out of four participants 15 years and older have an abnormally high serum total cholesterol (23.9\%) and LDL cholesterol $(28.8 \%)$, and one out of two an abnormally low HDL cholesterol level $(47.9 \%)$. Hypercholesterolemia is a major risk factor for cardiovascular disease. Statins are the most widely used drugs to lower blood cholesterol in people with hypercholesterolemia. Muscle symptoms such as pain (myalgia), myopathy and rhabdomyolysis are the most common side effects of long term statin treatment. Statin-related muscle symptoms (pain, weakness and injury) may be significantly exacerbated by intense physical activity and these symptoms are further enhanced by age. An alternative treatment for athletes with hypercholesterolemia is thus warranted in many cases. Bioactive oat beta-glucan has been proven to reduce blood cholesterol levels. High cholesterol is a risk factor in the development of coronary heart disease. A European Food Safety Authority (EFSA) article 14 claim exists for oat beta-glucans inferring a reduction in the risk of heart disease. This benefit is achieved with a daily intake of $3 \mathrm{~g}$ bioactive oat beta-glucan. Additionally, an EFSA article 13.1 approval exists for oat beta-glucans for blood glucose control and gut health. Oat beta-glucan has been shown to prolong glucose absorption which may affect energy supply in endurance exercise, may increase time to exhaustion and recovery from fatigue. Oat beta-glucan should thus be considered in terms of cholesterol management, glucose control and gut health for athletes

Riaan van der Schyff

Affiliation: None

Additional Authors: Riaan van der Schyff, Yoga Coopoo

\section{An Analysis of Corporate On-Site Fitness Facility Models Used in Selected Banking Establishments in South Africa}

Introduction: There has been a growing interest in employee wellness programmes at South African companies, as well as in on-site fitness facilities offered as part of this employee value proposition. While there are similarities between this type of fitness facility and private or commercial facilities, the operating environment for on-site facilities is vastly different. The decision between insourcing and outsourcing the management of such a facility is very important, especially since a specific skill set and approach is necessary for this task.

Exercise and physical activity have been proven to be effective in increasing productivity, lowering stress levels and improving the long-term health outcomes of employees. The question is, however: what management practices are currently used for such facilities to ensure the best possible outcomes for employees who are members of the facilities?

Methods: The management of four on-site fitness facilities at banks in Gauteng was compared based on several aspects including management of daily operations, the positioning of the service in the greater company EWP and satisfaction and the health outcomes of consumers ( $\mathrm{n}=626)$ at the facilities. The consumers' experience of facility management was compared to managers' perspectives.

Findings: Users of outsourced facilities were more satisfied with the state of the facilities compared to insourced facility users, although this did not influence health outcomes as no difference was found between the two groups. There was also an inverse relationship between facility fees and number of enrolled members.

Management approaches did not show distinct differences between the two models; rather, clear differences were shown when considering the funding models of the facilities. Some funding from the company is necessary for these facilities to be viable. Insourced facility managers had strategic input in employee wellness programs.

Conclusion: On-site fitness facilities can act as strategic levers for company employee wellness programmes and contribute to realisation of positive programme outcomes thereby impacting productivity and company bottom lines. 


\title{
The role of parents regarding their Support and KnowledgeTowards the Use of Nutritional Supplements
}

Introduction: The use of Nutritional Supplements (NS) by adolescents seem to be an escalating problem In South Africa. The role of parents seem to be questionable as children are allowed to use NS. Despite information websites by organisations like SAIDS and information sessions arranged by schools, parents do not seem to realize the health risks. For the purpose of this study, NS refer to any supplementary product ingested to boost the nutritional content of a normal diet to either fill a presumed need or deficiency. This could include any sport- or energy drinks, tablets, powders or injections that an athlete consumes as energy- or performance enhancement supplements. It includes any pharmacological or nutritional aids used in the hope of improving performance.

Objectives: In view of the lack of literature investigating the role of parents in the use of NS by their children in South Africa and internationally, the objective of the study was to determine the attitudes and level of knowledge of parents of children on the East Rand towards NS usage.

Methods: The design of the study was cross-sectional and used a self-administered questionnaire as well as an interview with a focus group. A sample of participants were obtained from parents of high school athletes involved in open teams in selected sport codes. Participants were invited to participate voluntarily and anonymously in completing the questionnaires. The sample of participants in the focus group were obtained from coaches involved in selected open teams from the same high schools. The data was analysed using largely descriptive statistics.

Results: Nine percent of parents indicated that they were well informed regarding NS. Sixty nine percent believed that NS acted as a gateway drug and $64 \%$ indicated that they would support their children in obtaining NS without efficacy being proven. Seventy five percent of parents indicated their awareness of the risk of NS being contaminated. Seventy three percent of parents deem their role in the athlete-coach-parent triangle as important yet only $13 \%$ indicated that their children rely on them for knowledge regarding NS. Coaches viewed the role of parents as integral in a healthy diet as they provide the means to eating habits, and indicated that the use of NS could not be ignored and needed to be acknowledged to build a good coachathlete relationship. Coaches believed that more expensive NS were better products to suggest for usage yet at the same time they also admitted a lack of knowledge regarding NS.

Conclusion: The role of parents are deemed as very important by coaches. Both parties demonstrated limited knowledge regarding NS. Parents have a positive attitude towards the use of NS by their children despite indicating that there are health risks related to the usage of NS. Parents considered the success of their children in sport performance as important for future development of their children.

\author{
Lynn van Rooy \\ Affiliation: University of Johannesburg \\ Additional Authors: Lynn van Rooy, Yoga Coopoo
}

\section{The change in knowledge regarding nutrition in coronary artery bypass graft patients}

Introduction: In order to reduce coronary artery disease (CAD) risk, moderate physical activity should be combined with other lifestyle modifications, such as proper nutrition, to have a dramatic impact. This necessitates educational and preventative measures, which should begin in childhood and continue throughout life. The aim of this study was to measure the change in nutrition knowledge of coronary artery bypass graft patients by implementation of a lifestyle intervention programme.

Methods: The Hawkes and Nowak Nutrition Knowledge Questionnaire (1998) was

administered to 18 coronary artery bypass graft (CABG) patients to assess the change in nutrition knowledge.

Results: Significant improvements were noted in the nutrition knowledge score $(18.9 \pm 3.4$ to $23.2 \pm 4.5 ; p=0.000)$. Although all components measured exhibited improvements in knowledge, cholesterol reduction knowledge $(5.3 \pm 1.8$ to $7.2 \pm 1.8 ; p=0.0066)$, low fat food knowledge ( $3.8 \pm 2.3$ to $5.1 \pm$ $2.7 ; p=0.011)$ and high fibre food knowledge $(4.1 \pm 1.4$ to $4.7 \pm 1.1 ; p=0.022)$ exhibited the highest and most significant improvements.

Conclusion: Notably, these significant improvements in nutrition knowledge points toward effective education being delivered during the intervention.

Keywords: Coronary artery disease, CABG, nutrition, cardiac rehabilitation, lifestyle modification.

Waeil Ali Mohammed Yahya Waeil

Affiliation: Sport physiotherapy

Additional Authors: Waeil Ali Mohammed, Yahya Waeil, Mohammed Saeed Alkhleefa

\section{Neurological pain in football players simulating groin pain.cupping test stored the problem}

Introduction: Groin pain is a common problem found in many athletes. Many athletes will present to clinicians with a history of groin pain that may be acute or chronic in nature, and may stem from a single traumatic event or repeated microtrauma to the region or it may be due to Nerve roots damage spine could cause radicular symptoms referred to the pelvis or groin. Sudan-cupping test is a newly applied and newly registered dry cupping test, based on the use of different sized cups for cupping over certain areas of spine. The test can detect the radiating pain to the joints (knee, ankle or shoulder) and muscles.

Methodology: 500 Professional football players at the age between 17 and 30 years were examined for groin pain for the period 2012-2014. Players are examined to detect symptoms that suggest some problem with the nerves (numbness, tingling, weakness or pain).Sudan cupping test is applied to detect radiating pain from the lumber spine. Positive Sudan cupping test are sent for nerve conduction study to confirm the presence of nerve roots damage.

Results: 61 players with groin pain. 39 chronic and 22 acute cases were found to have Sudan cupping and NCS positive results suggestive for nerve roots compression. They were diagnosed and treated as neurological groin pain injuries.

Conclusion: Diagnosis is the key for the treatment and rehabilitation of groin pain. Sudan cupping resistant cases for treatment.it helps the players to return fast to the field test is helpful for the detection of the origin of the groin pain. This decreases the number of. 


\section{Non-supportive Touch improves Postural Sway in Active and Inactive Individuals with Parkinson's Disease}

Objective: To determine if activity status influences static postural stability (PS) with and without non-supportive light manual touch (LMT) in mild to moderate Parkinson's Disease (PD).

Background: PD individuals demonstrates balance impairment and a high incidence of fall-related injuries, which is associated with inactivity and reduced quality of life. Inactivity still remains one of the greatest problems in PD, although exercise has shown to be beneficial in improving functional abilities and reduce fall risk. Therefore it is vital to investigate cost effective non-pharmacological interventions to improve balance. Moreover it is believed that PD individuals have diminished proprioception due to impaired sensory integration and increased reliance on visual feedback for balance. However, recently researchers reported that touch, which is independent of mechanical support, may improve static PS via tactile feedback.

Methods: Relative smoothness (JERK), amplitude (RMS), mean velocity (MV) with a tri-axial accelerometer and perceived stability were assessed during a static balance task under 6 sensory conditions. Participants completed a modified-tandem standing task under eyes open (EO), eyes closed (EC), unrestricted manual contact (UMC), LMT only and no manual contact (NMC) as well as on (+F) or off a foam pad conditions.

Results: Mean age for 9 active (ACT) and 7 inactive participants were 67 (SD 8) and 70 (SD 4) years, respectively. Regardless of the sensory condition ACT tend to have less JERK and RMS ( $p=0.06$ ). Groups presented less PS during all LMT conditions compared to NMC ( $<<0.01)$ and UMC ( $<<0.01)$. EC LMT+F differed between the groups for JERK ( $p<0.001$ ) and RMS ( $p=0.03$ ), with ACT showing $154 \%$ and $40 \%$ less JERK and RMS, respectively. Medial Lateral MV was $44 \%$ slower in the ACT during EC NMC ( $p=0.04)$ compared to inactive. Groups felt more successful when they received LMT compared to NMC $(p<0.01)$

Conclusions: Non-supportive LMT may be more effective in reducing PS than UMC in both active and inactive PD individuals. Suggesting that nonsupportive LMT can be used to improve static balance in individuals with mild to moderate PD. In addition, active individuals demonstrated less static postural instability compared to inactive individuals. Tactile feedback activities could help individuals to maintain PS enabling them to perform daily activities safely with a decreased fall risk. 